UCLA/08/TEP/17

LAPTH-1268/08

8 November 2008

\title{
Half-BPS Supergravity Solutions and Superalgebras ${ }^{1}$
}

\author{
Eric D’Hoker, John Estes², Michael Gutperle, Darya Krym \\ Department of Physics and Astronomy \\ University of California, Los Angeles, CA 90095, USA \\ Paul Sorba \\ LAPTH, ${ }^{3}$ Université de Savoie, CNRS; \\ 9, Chemin de Bellevue, BP110, F-74941 Annecy-le-Vieux, Cedex, France.
}

\begin{abstract}
We establish a correspondence between certain Lie superalgebras with 16 fermionic generators, and half-BPS solutions to supergravities with 32 supersymmetries. Three cases are discussed. For Type IIB supergravity, we relate semi-simple Lie superalgebras $\mathcal{H}$ with 16 fermionic generators which are subalgebras of $P S U(2,2 \mid 4)$, to families of half-BPS solutions which are invariant under $\mathcal{H}$, and locally asymptotic to $A d S_{5} \times S^{5}$. Similarly, for M-theory, we relate semi-simple Lie superalgebras $\mathcal{H}$ with 16 fermionic generators which are subalgebras of $O S p\left(8^{*} \mid 4\right)$ or $O S p(8 \mid 4, \mathbf{R})$ to families of half-BPS solutions which are invariant under $\mathcal{H}$, and locally asymptotic to $A d S_{7} \times S^{4}$ or $A d S_{4} \times S^{7}$ respectively. Possible enhancements to more than 16 supersymmetries, such as 24 , are also analyzed. The classification of semi-simple subalgebras of $P S U(2,2 \mid 4), O S p\left(8^{*} \mid 4\right)$, and $O S p(8 \mid 4, \mathbf{R})$ derived in this paper, leads us to conjecture the existence of various new families of half-BPS solutions to Type IIB supergravity and M-theory.
\end{abstract}

\footnotetext{
${ }^{1}$ This work was supported in part by NSF grants PHY-04-56200 and PHY-07-57702.

${ }^{2}$ Present address: Centre de Physique Théorique, Ecole Polytechnique, F-91128 Palaiseau, France.

${ }^{3}$ Laboratoire de Physique Théorique d'Annecy-le-Vieux, UMR 5108.

E-mail addresses: dhoker@physics.ucla.edu; johnaldonestes@gmail.com; gutperle@physics.ucla.edu; dk320@physics.ucla.edu; sorba@lapp.in2p3.fr.
} 


\section{Introduction}

Supersymmetry places powerful constraints on the existence of solutions to supergravity. Invariance of a solution under supersymmetry is accompanied by invariance under bosonic symmetries, such as isometries, which result from the composition of supersymmetry transformations. The isometries strongly constrain the space-time manifold, metric, and other fields of the solution. Together, supersymmetries and bosonic symmetries close into a Lie superalgebra, under which the solution is said to be invariant.

In 10-dimensional Type IIB supergravity and in 11-dimensional M-theory, the maximal number of supersymmetries preserved by any solution is 32 . The only solutions with maximal supersymmetry correspond to the following space-time manifolds and Lie superalgebras,

$\begin{array}{lll}\text { Type IIB } & A d S_{5} \times S^{5} & P S U(2,2 \mid 4) \\ \text { M-theory } & A d S_{7} \times S^{4} & O S p\left(8^{*} \mid 4\right) \\ \text { M-theory } & A d S_{4} \times S^{7} & O S p(8 \mid 4, \mathbf{R})\end{array}$

and their respective flat Minkowski space-time and plane-wave limits [1, 2, 3]. Solutions invariant under 28 or more supersymmetries have been shown to automatically possess maximal supersymmetry [4]. With fewer than 28 supersymmetries, many solutions have been constructed explicitly, but no general classification is available.

The goal of the present paper is to classify, in Type IIB supergravity and M-theory, solutions which are invariant under at least 16 supersymmetries (so-called half-BPS solutions), and are subject to certain asymptotic conditions, to be spelled out below. The ultimate goal will be to obtain these solutions in exact form, but this will be left for future work.

Various half-BPS solutions have long played a prominent role in string theory already, including the F1/D1, D3, NS5/D5, and D7 branes in Type IIB, and the M2 and M5 branes in M-theory. Certain intersections of branes preserve just 8 supersymmetries (so-called quarter$B P S$ solutions), but these may be enhanced to 16 supersymmetries in the near-horizon limit, thereby yielding further half-BPS solutions. The AdS/CFT correspondence maps these halfBPS solutions onto half-BPS chiral operators in the dual conformal field theory.

Over the past few years, a wealth of half-BPS solutions have been derived in exact form. They include the exact solutions of [5] which are AdS/CFT dual to local half-BPS gauge invariant operators in Type IIB and in M-theory [6]. In Type IIB, they include the exact solutions of $[7,8]$ and of [9], which are AdS/CFT dual respectively to a half-BPS planar interface and to a half-BPS Wilson line in $\mathcal{N}=4$ super Yang-Mills theory (SYM). In Mtheory, they include the exact solutions of [10], which are dual to a half-BPS line-interface 
and a half-BPs boundary operator respectively in the associated $2+1$ - and 5+1-dimensional CFTs. In all these cases, one actually has families of solutions described by non-trivial moduli spaces, which may be related to the near-horizon limits of quarter-BPS localized brane intersections.

A general program aimed at classifying the local form of supersymmetric solutions, in terms of Killing spinor bilinears and $G$-structures, was proposed in [11, 12] (for a review, see [13]). These methods have since been widely applied to supersymmetric AdS-type solutions, beginning in [14], and in subsequent papers, many of which will be mentioned later.

\subsection{The half-BPS solution - superalgebra correspondence}

Given the special importance of half-BPS solutions to string theory and to the AdS/CFT correspondence, it will clearly be of value to obtain their complete classification. In this paper, we shall propose a solution to this problem in terms of Lie superalgebras.

Specifically, we shall exhibit a correspondence between a Lie superalgebra $\mathcal{H}$ which is a subalgebra with 16 fermionic generators of $\operatorname{PSU}(2,2 \mid 4)$, and a family of half-BPS solutions to Type IIB supergravity whose asymptotics at space-time infinity approaches $A d S_{5} \times S^{5}$. We exhibit a similar correspondence between a subalgebra $\mathcal{H}$ with 16 fermionic generators of $O S p\left(8^{*} \mid 4\right)$ or $O S p(8 \mid 4, \mathbf{R})$ and a family of half-BPS solutions to M-theory whose asymptotics at space-time infinity approaches respectively $A d S_{7} \times S^{4}$ or $A d S_{4} \times S^{7}$. For simplicity, we shall restrict to subalgebras $\mathcal{H}$ which are basic Lie superalgebras or direct sums thereof. ${ }^{1}$

More precisely, we shall argue that a half-BPS solution of Type IIB supergravity which is asymptotic to $A d S_{5} \times S^{5}$ must be invariant under a superalgebra with 16 fermionic generators $\mathcal{H}$ which is a subalgebra of $P S U(2,2 \mid 4)$ (and analogously for M-theory). We shall show that there exist a finite number of such subalgebras $\mathcal{H}$ so that, as a result, this correspondence provides a classification of the half-BPS solutions with the above asymptotics. All cases of known exact solutions of Type IIB supergravity and M-theory with respectively $A d S_{5} \times S^{5}$ and $A d S_{7} \times S^{4}$ or $A d S_{4} \times S^{7}$ asymptotic behavior indeed obey this correspondence.

A classification of all basic Lie superalgebras $\mathcal{H}$ with 16 fermionic generators contained in $P S U(2,2 \mid 4), O S p\left(8^{*} \mid 4\right)$, and $O S p(8 \mid 4, \mathbf{R})$ did not appear to be available prior to this work, and will thus be derived here. In particular, we shall show that none of the exceptional Lie

\footnotetext{
${ }^{1}$ Basic Lie superalgebras will be defined in Section 4; they are the simple Lie superalgebras closest in properties to the ordinary simple Lie algebras. It would be interesting to study also superalgebras $\mathcal{H}$ which are non-semi-simple (such as those arising from the contraction of semi-simple superalgebras in the PP-wave problem), or which include the Lie superalgebra $P S U(1 \mid 1)$, the "strange" classical superalgebras $P(n)$ and $Q(n)$, or those of "Cartan type", $W(n), S(n), \tilde{S}(n)$, and $H(n)$, but this will not be done here.
} 
superalgebras, $F(4), G(3)$, or $D(2,1 ; c)$ with $c \neq 1,-2,-1 / 2$, or any of their real forms, is a subalgebra of $P S U(2,2 \mid 4), O S p\left(8^{*} \mid 4\right)$ or $O S p(8 \mid 4, \mathbf{R})$.

As a result, in Type IIB supergravity, no solutions exist which are asymptotic to $A d S_{5} \times S^{5}$ and invariant under a basic Lie superalgebra, or direct sum of basic Lie superalgebras, $\mathcal{H}$, which is not in the above classification (e.g. when $\mathcal{H}$ is an exceptional superalgebra). The analogous result holds for M-theory. The converse of the correspondence, namely whether every regular solution to Type IIB supergravity invariant under a basic Lie superalgebra $\mathcal{H}$ with 16 fermionic generators contained in $P S U(2,2 \mid 4)$ must be asymptotic to $A d S_{5} \times S^{5}$ (and analogously for M-theory), appears not to hold.

All known cases of exact solutions actually consist of families, with solutions depending on a number of free parameters, referred to as moduli. To each basic Lie superalgebra, or direct sum of basic Lie superalgebras, $\mathcal{H}$, with 16 fermionic generators, which is a subalgebra of $P S U(2,2 \mid 4), O S p\left(8^{*} \mid 4\right)$, or $O S p(8 \mid 4, \mathbf{R})$, we associate a moduli space $\mathcal{M}_{\mathcal{H}}$ of regular solutions with the above asymptotics for each respective supergravity. The solution with 32 supersymmetries always trivially belongs to $\mathcal{M}_{\mathcal{H}}$. For all the non-trivial known cases, $[5,8,7,9,10]$, the moduli spaces display non-trivial topology and geometry, and it becomes an interesting mathematical question to characterize $\mathcal{M}_{\mathcal{H}}$ for each $\mathcal{H}$. In particular, the connectedness properties of $\mathcal{M}_{\mathcal{H}}$ will reveal to what degree solutions are continuously connected to the maximally symmetric solution.

These moduli spaces may contain subvarieties where supersymmetry is enhanced, and the number of supersymmetry generators is larger than 16, but smaller than 28 . We shall also classify these possible solutions via their invariance superalgebras.

For the cases of superalgebras $\mathcal{H}$ where no non-trivial half-BPS solutions are known yet, we shall compare arguments based on intersecting and probe branes, the CFT duals, superalgebra theory, and the structure of the Ansatz for supergravity fields to argue either for the existence or for the absence of new non-trivial half-BPS solutions. The list of all cases

produced by our proposed correspondence between Lie superalgebras and half-BPS solutions for Type IIB will be given in Table 10, and for M-theory in Tables 11 and 12.

\subsection{Half-BPS solutions related to D7 branes}

Cases related to D7 branes in Type IIB merit to be highlighted here. On the one hand, the existence of near-horizon limits involving intersections of D7 branes with D3 branes, or of D7 branes viewed as probes in the $A d S_{5} \times S^{5}$ background, suggests that fully back-reacted half-BPS solutions can exist. On the other hand, D7 branes produce flavor multiplets in the dual CFT, which generate a non-vanishing $\beta$-function, and thus vitiate solutions with $A d S_{3}$ 
or $A d S_{5}$ factors on the gravity side. Arguments have been presented in [15] and in [16, 17] (following earlier work on D7 branes in $[18,19]$ ), that no fully back-reacted near-horizon limit solutions corresponding to D7 branes should exist.

Our analysis will reveal the existence of two cases (namely II* and III* of Table 10) corresponding to the superalgebras $S U(1,1 \mid 4) \oplus S U(1,1)$ and $S U(2,2 \mid 2) \oplus S U(2)$ which are subalgebras of $P S U(2,2 \mid 4)$, and whose global symmetries and space-time structure precisely match those of the D7 probe or D7/D3 intersecting brane analysis. These cases correspond respectively to D7/D3 intersections of dimensions 2 or 4 . Closer analysis of the superalgebra structure reveals, however, further layers of subtlety (see section 5.4). Each subalgebra, $S U(1,1 \mid 4) \oplus S U(1,1)$ or $S U(2,2 \mid 2) \oplus S U(2)$, possesses a purely bosonic invariant subalgebra (respectively $S U(1,1)$ and $S O(3)$ ), which is not needed for the half-BPS condition, and thus not protected by supersymmetry. Arguments, to be presented in subsection 5.4, will indicate that these extra bosonic invariant subalgebras are incompatible with asymptotic $A d S_{5} \times S^{5}$ behavior (though compatible with local asymptotic behavior). Removing these bosonic factors leads to corresponding "general cases", namely II and III of Table 10, for which superalgebra arguments suggest the existence of solutions. The general cases II and III now have, however, insufficient symmetry to support fully back-reacted half-BPS solutions for the near-horizon limit of D7/D3 branes.

\subsection{Families of half-BPS solutions in M-theory}

The correspondence may be further illustrated by the M-theory solutions of [20] (see also $[21,22])$ and the M-theory solutions of [10], which both form families of solutions invariant under the exceptional Lie superalgebra $\mathcal{H}=D(2,1 ; c ; 0) \oplus D(2,1 ; c ; 0)$. Here, $c \neq 0$ is a free real parameter, and the last entry "0" in $D(2,1 ; c ; 0)$ refers to the real form of $D(2,1 ; c)$ whose maximal bosonic subalgebra is $S O(1,2) \oplus S O(4)$. For $c=-2$ or $c=-1 / 2, \mathcal{H}$ is a subalgebra of $O S p\left(8^{*} \mid 4\right)$ and the exact solutions of [10] are locally asymptotic to $A d S_{7} \times S^{4}$. Similarly, for $c=1, \mathcal{H}$ is a subalgebra of $\operatorname{OSp}(8 \mid 4, \mathbf{R})$ and the associated exact solutions of [10] are locally asymptotic to $A d S_{4} \times S^{7}$. Finally, for $c \neq 1,-2,-1 / 2, \mathcal{H}$ is a subalgebra of neither $O S p\left(8^{*} \mid 4\right)$ nor $O S p(8 \mid 4, \mathbf{R})$, and it has been argued in [10] that the local asymptotics of the solutions can be neither $A d S_{7} \times S^{4}$ nor $A d S_{4} \times S^{7}$. The solutions of [20,21] have a space-time manifold of the form $A d S_{3} \times S^{3} \times S^{3} \times E_{2}$ for all values of $c$. Here, the product of the space-time factors is genuinely direct, so that no warping occurs over the flat surface $E_{2}$ with boundary. In particular, for $c=1,-2,-1 / 2$, there exist solutions asymptotic to $A d S_{3} \times S^{3} \times S^{3} \times E_{2}$, as well as to $A d S_{4} \times S^{7}$ or $A d S_{7} \times S^{4}$, which indicates that the converse of the correspondence does not hold. 


\subsection{Organization}

The remainder of this paper is organized as follows. In Sections 2 and 3, we review known constructions of half-BPS configurations in respectively Type IIB and M-theory from the points of view of their CFT duals, probe and intersecting branes, and exact supergravity solutions. In Section 4, we produce a classification of all semi-simple basic Lie superalgebras which are subalgebras of $P S U(2,2 \mid 4), O S p\left(8^{*} \mid 4\right)$, or $O S p(8 \mid 4, \mathbf{R})$. In Section 5, we apply the results of Section 4 towards the classification of old and new half-BPS solutions. In Section 6, conformal superalgebras [23] with 16 supersymmetries in various dimensions are matched with the superalgebras $\mathcal{H}$ which arise in our classification. In Section 7, a discussion is presented of when supersymmetry of half-BPS solutions may be enhanced in a non-trivial way, and how the M-theory solutions of [10] illustrate the correspondence. In Appendix A, relevant facts of ordinary Lie algebras are reviewed. In Appendix B, subalgebra relations between non-exceptional basic Lie superalgebras are proven. In Appendix C, it is demonstrated that none of the exceptional Lie superalgebras is a subalgebra of $P S U(2,2 \mid 4), O S p\left(8^{*} \mid 4\right)$, or $O S p(8 \mid 4, \mathbf{R})$. Finally, in Appendix D, we derive the superalgebra of the M-theory solution of [10]. Throughout, our analysis will be carried out at the level of Lie superalgebras rather than supergroups, thus leaving possible global issues for later investigation. 


\section{Half-BPS configurations in Type IIB}

Before initiating the classification of subalgebras of $P S U(2,2 \mid 4), O S p\left(8^{*} \mid 4\right)$, and $O S p(8 \mid 4, \mathbf{R})$ in Section 4, and its application to half-BPS solutions in Section 5, we begin by assembling evidence for the existence of various families of half-BPS solutions, by drawing together arguments from the AdS/CFT correspondence, from intersecting and probe brane dynamics, and from existing families of exact solutions.

\subsection{The AdS/CFT correspondence for Type IIB}

The AdS/CFT correspondence provides a map between Type IIB superstring theory on $A d S_{5} \times S^{5}$ and 4-dimensional $\mathcal{N}=4 \mathrm{SYM}$ with gauge group $S U(N)$ in its conformal phase [24]. The global symmetries, including the supersymmetries, on the AdS side match those on the CFT side, and are described by the superalgebra $P S U(2,2 \mid 4)$, whose maximal bosonic subalgebra is $S U(2,2) \oplus S U(4)=S O(2,4) \oplus S O(6)$. On the CFT side, $S O(2,4)$ is the 4dimensional conformal algebra, and $S U(4)$ is the R-symmetry. On the AdS side, $S O(2,4) \oplus$ $S O(6)$ is the isometry algebra of $A d S_{5} \times S^{5}$ space-time, and the fermionic generators are associated with 32 Killing spinors of this maximally supersymmetric solution. Furthermore, there exists an explicit map between correlators of gauge invariant operators in $\mathcal{N}=4$ SYM theory (in the 't Hooft limit, and large 't Hooft coupling) and solutions to Type IIB supergravity subject to suitable boundary conditions [25, 26] (for reviews, see [27], and [28]).

The CFT vacuum has maximal symmetry, given by the superalgebra $P S U(2,2 \mid 4)$, and corresponds to the maximally symmetric $A d S_{5} \times S^{5}$ solution on the AdS side. Inserting an operator $\mathcal{O}$ in the $\mathrm{CFT}$ vacuum produces a new state whose symmetry is reduced to the subalgebra $\mathcal{H}$ of $P S U(2,2 \mid 4)$ that leaves $\mathcal{O}$ invariant. The insertion of $\mathcal{O}$ on the CFT

side causes the Type IIB solution to deform away from $A d S_{5} \times S^{5}$ on the AdS side. If the operator $\mathcal{O}$ is "sufficiently local", then there will exist an asymptotic bulk region in $\mathbf{R}^{4}$, away from the support of $\mathcal{O}$, where locally the state of the CFT is close to the vacuum state. The asymptotics at space-time infinity of the AdS dual supergravity solution then coincides locally with $A d S_{5} \times S^{5}$. Here, "sufficiently local" operators include, for example, those whose support in $\mathbf{R}^{4}$ is at least co-dimension 1 , which is the case of local operators, Wilson loops, interface operators, and surface operators.

This AdS/CFT argumentation shows that any half-BPS solution which is AdS/CFT dual to a "sufficiently local" operator in the CFT will obey the local asymptotics of $A d S_{5} \times S^{5}$, and will be invariant under a subalgebra $\mathcal{H}$ with 16 fermionic generators of $P S U(2,2 \mid 4)$. 


\subsection{Half-BPS operators in $\mathcal{N}=4 \mathrm{SYM}$}

In this subsection we shall review half-BPS gauge invariant operators in $\mathcal{N}=4 \mathrm{SYM}$. It is customary to distinguish between local and non-local operators. By the state operator mapping, local operators are associated with superconformal chiral primary states of the CFT. Examples of non-local operators include the Wilson loop, the 't Hooft loop, surface operators, and interface/defect operators. Non-local operators are useful as probes and often provide order parameters which can distinguish the different phases of the theory.

- A local half-BPS operator forms a multiplet associated with a chiral primary state, which can be constructed as follows. The scalar fields of $\mathcal{N}=4 \mathrm{SYM}$ will be denoted $\Phi^{i}$, with $i=1, \cdots, 6$, and the gauge field by $A_{\mu}$, both of which transform under the adjoint representation of the $S U(N)$ gauge group. One considers the theory on $\mathbf{R} \times S^{3}$, and chooses a complex combination $Z=\Phi^{1}+i \Phi^{2}$ of the scalars. The scalar field $Z$ carries a charge $J=1$ under the $U(1)$ subalgebra of the $S U(4)$ R-symmetry corresponding to rotations of scalars in the $1-2$ plane. The chiral primary state is given by a multi-trace operator,

$$
\mathcal{O}=\prod_{i} \operatorname{tr}\left(Z^{n_{i}}\right) \quad J=\sum_{i} n_{i}
$$

The conformal dimension $\Delta$ saturates the BPS bound, and we have $\Delta=J$. The operator $\mathcal{O}$ is invariant under time translations and $S O(4)$ rotations of $S^{3}$. The particular linear combination chosen to define $Z$ in terms of $\Phi^{i}$ breaks the $S U(4)$ R-symmetry to its $S O(4)$ subalgebra. In total, the operator (2.1) is invariant under $\mathbf{R} \oplus S O(4) \oplus S O(4)$ and preserves 16 supersymmetries, enlarging the symmetry to a $\mathbf{R} \oplus P S U(2 \mid 2) \oplus P S U(2 \mid 2)$ superalgebra.

- A supersymmetric Wilson loop is a non-local operator associated with a curve $\mathcal{C}$ in $\mathbf{R}^{1,3} \times \mathbf{R}^{6}$ parameterized by $\left\{x^{\mu}(\tau), y^{i}(\tau)\right\}$, where $\mu=0,1,2,3$ and $i=1, \cdots, 6$,

$$
W(R, \mathcal{C})=\operatorname{tr}_{R}\left\{P \exp i \oint_{\mathcal{C}}\left(A_{\mu} d x^{\mu}+\Phi^{i} d y^{i}\right)\right\}
$$

Here, $P$ stands for path ordering the exponential, and the $\operatorname{trace} \operatorname{tr}_{R}$ is to be evaluated in a representation $R$ of the gauge group $S U(N)$. It has been shown in [29,30] that a Wilson loop preserves 16 supercharges if the curve $\mathcal{C}$ is a straight line, given by $x^{0}=\tau, x^{1}=x^{2}=x^{3}=0$, and $y^{i}=n^{i} \tau$ where $n^{i}$ is a vector of unit norm in $\mathbf{R}^{6}$. Such a Wilson loop breaks the bosonic $S O(2,4) \oplus S O(6)$ symmetry to $S O(2,1) \oplus S O(3) \oplus S O(5)$. The superalgebra preserved by the Wilson loop is $O S p\left(4^{*} \mid 4\right)$.

- A surface operator is associated with a 2-dimensional surface $\mathcal{S}$ in $\mathbf{R}^{1,3}$. In $\mathcal{N}=4$ SYM it may be defined $[31,32]$ as a disorder operator which prescribes a codimension 2 
singularity for the YM fields along the surface $\mathcal{S}$. The surface operator is therefore a higherdimensional analog of the 't Hooft loop in the sense that neither can be expressed as a path integral insertion using the local fields that appear in the Lagrangian. A surface operator is half-BPS if $\mathcal{S}=\mathbf{R}^{1,1}$ and the singularity along $\mathcal{S}$ selects a single complex scalar field $Z$. The bosonic symmetries are the conformal $S O(2,2)$ symmetry of the surface world-volume, and the $S O(4)$ R-symmetry unbroken by the choice of $Z$. The superalgebra preserved by a surface operator is $P S U(1,1 \mid 2) \oplus P S U(1,1 \mid 2) \oplus U(1)$, see $[33,34]$.

- A defect/interface operator is associated with a $2+1$-dimensional space $\mathcal{D}$ in $\mathbf{R}^{1,3}$. Boundary conditions for the SYM fields, and possible worldvolume actions which preserve 16 supersymmetries were analyzed in $[35,36,37,38]$. It was shown that if $\mathcal{D}$ is chosen to be $\mathcal{D}=\mathbf{R}^{1,2}$ and the $S U(4)$ R-symmetry is broken to $S U(2) \oplus S U(2)$, the conformal algebra $S O(2,3)$, as well as 16 supersymmetries, are unbroken by the defect/interface. This half-BPS planar defect/interface preserves the superalgebra $O S p(4 \mid 4, \mathbf{R})$.

\subsection{Intersecting branes in flat space-time}

A stack of parallel D3-branes in flat space-time preserves 16 of the 32 supersymmetries. More generally, the unbroken supersymmetries of a Dp-brane in flat space are given by

$$
Q=Q_{L}+\prod_{i} \Gamma^{i} Q_{R}
$$

where the index $i$ runs over all space-time direction transverse to the Dp-brane world-volume.

\begin{tabular}{|c|c|c|c|c|}
\hline Case & branes & dim intersec & $\#_{N D}$ & bosonic symmetry \\
\hline \hline 1 & D3/D1 & 1 & 4 & $\mathbf{R} \oplus S O(3) \oplus S O(5)$ \\
\hline 2 & D3/D5 & 1 & 8 & $\mathbf{R} \oplus S O(3) \oplus S O(5)$ \\
\hline 3 & D3/D3 & 2 & 4 & $I S O(1,1) \oplus S O(4) \oplus S O(2)$ \\
\hline 4 & D3/D7 & 2 & 8 & $I S O(1,1) \oplus S O(6) \oplus S O(2)$ \\
\hline 5 & D3/D5 & 3 & 4 & $I S O(2,1) \oplus S O(3) \oplus S O(3)$ \\
\hline 6 & D3/D7 & 4 & 4 & $I S O(3,1) \oplus S O(4) \oplus S O(2)$ \\
\hline \hline
\end{tabular}

Table 1: Intersecting D3/Dp branes in Type IIB preserving 8 supersymmetries

The intersection of a Dp and a Dp' brane preserves 8 of the 32 supersymmetries if the number $\#_{N D}$ of Neumann-Dirichlet directions (i.e. the directions which are transverse to one and longitudinal to the other brane, but not both) is either 4 or 8 . In Table 1, 
we list all supersymmetric intersections of a D3 brane with a Dp brane which preserve 8 supersymmetries. The cases are ordered by increasing dimensionality of the intersection. ${ }^{2}$

In cases 1, 2, and 5 it is known that the near-horizon limit will enhance the number of unbroken supersymmetries from 8 to 16 . In addition, the Poincaré symmetry $I S O(1, d-1)$ will be enhanced to the conformal symmetry $S O(2, d)$. In the absence of fully localized supergravity solutions for the intersecting branes in cases 3, 4, and 6, it remains an open question whether the Poincaré invariance and supersymmetry will be enhanced in those cases, particularly the ones involving D7 branes (see also the subsequent subsection).

\subsection{Probe branes in $A d S_{5} \times S^{5}$}

A different approach to investigating half-BPS brane configurations starts from the $A d S_{5} \times S^{5}$ near-horizon limit of D3 branes and considers inserting supersymmetric probe branes. In the probe approximation, the strength of the back-reaction of the probe brane onto the geometry is neglected. The back-reaction of the probe brane is governed by $g_{s} N_{\text {probe }}$, where $g_{s}$ is the string coupling constant and $N_{\text {probe }}$ is the number of probe branes. In the 't Hooft limit, where $g_{s} \rightarrow 0, N \rightarrow \infty$ with $\lambda=g_{s} N$ held fixed, the back-reaction is negligible for finite $N_{\text {probe }}$. On the field theory side this is reflected by the fact that the world-volume theory on the probe is in general not conformal and that the $\beta$-function is proportional to $g_{Y M}^{3} N_{\text {probe }}$ which vanishes in the 't Hooft limit for finite $N_{\text {probe }}$.

Consistency of the probe brane requires that it solve the equations of motion for the embedding of the probe into $A d S_{5} \times S^{5}$, derived from the Dirac-Born-Infeld world-volume action for the probe brane. The embedding of the probe $p$-brane is parameterized by a $p+1$ dimensional submanifold $M$ of $A d S_{5} \times S^{5}$. To describe this more explicitly, we represent $A d S_{5}$ as a hyperboloid in $\mathbf{R}^{2,4}$, and $S^{5}$ as a sphere in $\mathbf{R}^{6}$, both with the same radius $R$,

$$
\begin{aligned}
X_{0}^{2}+X_{5}^{2}-X_{1}^{2}-X_{2}^{2}-X_{3}^{2}-X_{4}^{3} & =R^{2} \\
Y_{1}^{2}+Y_{2}^{2}+\cdots+Y_{6}^{2} & =R^{2}
\end{aligned}
$$

We are interested in cases where the manifold $M$ is a product of an $A d S_{n}$ submanifold in $A d S_{5}$ parametrized by

$$
\begin{aligned}
X_{0}^{2}+X_{5}^{2}-X_{1}^{2}-X_{n-1}^{2} & =R^{2} \cosh ^{2} \rho \\
X_{n}^{2}+\cdots+X_{4}^{2} & =R^{2} \sinh ^{2} \rho
\end{aligned}
$$

\footnotetext{
${ }^{2}$ Although the bosonic symmetry is allowed to have an extra $S O(2)$, in addition to the bosonic symmetries listed, in case 3 of Tables 1, 2, 3, and 4, and in case 4 of Table 5, the generic solution has no such factor $[33,39]$. We thank Jaume Gomis for stressing this result to us.
} 
and an $S^{m}$ submanifold in $S^{5}$ parameterized by

$$
\begin{aligned}
Y_{1}^{2}+Y_{2}^{2}+\cdots Y_{m+1}^{2} & =R^{2} \cos ^{2} \alpha \\
Y_{m+2}^{2}+\cdots+Y_{6}^{2} & =R^{2} \sin ^{2} \alpha
\end{aligned}
$$

The Dirac-Born-Infeld equations of motion for the probe brane then determine the position $\rho$ of the $A d S_{n}$ inside $A d S_{5}$, and the position $\alpha$ of the $S^{m}$ inside the $S^{5}$. Note that the spheres $S^{m}$ have slipping modes, which tend to decrease their volume. Although one might think that such modes indicate an instability, they actually turn out to be above the BreitenlohnerFreedman bound [40], and hence do not introduce instabilities [41, 42, 43].

In Table 2 below, the possible supersymmetric Dp branes in $A d S_{5} \times S^{5}$ which are related to the brane intersections in flat space-time of Table 1 are listed in order of increasing world-volume dimension.

\begin{tabular}{|c|c|c|c|}
\hline Case & probe & worldvolume & bosonic symmetry \\
\hline \hline 1 & D1 & $A d S_{2}$ & $S O(2,1) \oplus S O(3) \oplus S O(5)$ \\
\hline 2 & D5 & $A d S_{2} \times S^{4}$ & $S O(2,1) \oplus S O(3) \oplus S O(5)$ \\
\hline 3 & D3 & $A d S_{3} \times S^{1}$ & $S O(2,2) \oplus S O(4) \oplus S O(2)$ \\
\hline 4 & D7 & $A d S_{3} \times S^{5}$ & $S O(2,2) \oplus S O(6) \oplus S O(2)$ \\
\hline 5 & D5 & $A d S_{4} \times S^{2}$ & $S O(2,3) \oplus S O(3) \oplus S O(3)$ \\
\hline 6 & D7 & $A d S_{5} \times S^{3}$ & $S O(2,4) \oplus S O(4) \oplus S O(2)$ \\
\hline \hline
\end{tabular}

Table 2: Probe Dp branes in $A d S_{5} \times S^{5}$ preserving 16 supersymmetries

The supersymmetry of the probe branes is established by checking the existence of a $\kappa$-symmetry projector for the Dp-brane world-volume action $[44,45,46]$. The projector may be used to gauge fix the world-volume action and to exhibit equal numbers of bosonic and fermionic degrees of freedom. It was shown in [41] that the probe branes in Table 2 are all supersymmetric.

Probe branes allow us to introduce additional structure into the AdS/CFT correspondence. In the probe approximation, the AdS/CFT duality is expected to "act twice" [42, 43], with the open strings on the probe branes being dual to the localized degrees of freedom on the intersection. Cases 1 and 2 have been discussed in [47]. Note that, in case 1, the D1-brane can be replaced by a "giant" D3-brane with the same symmetries and magnetic flux on its $A d S_{2} \times S^{2}$ worldvolume. Case 3 has been discussed in [39], where it was shown that a supersymmetric embedding preserves only one of the two $S O(2)$ symmetries. Case 4 has been discussed in the probe approximation in [16], and with the inclusion of the fully 
back-reacted geometry in [17]. Case 5 was argued to correspond to a holographic description of a defect conformal field theory $[42,43,48,49]$. Case 6 was used to introduce flavor into $\mathrm{AdS} / \mathrm{CFT}[50]$.

It is a priori not determined whether a probe solution can be extended to an exact supergravity solution with the same symmetries. The question whether including the gravitational back-reaction of the probe preserves all or destroys some of the symmetries of the configuration has to be decided on a case by case basis.

\subsection{Structure of known exact supergravity solutions}

The presently known exact supergravity solutions which preserve 16 supersymmetries share the following structure. We will focus on solutions which asymptotically approach $A d S_{5} \times S^{5}$. The Ansatz has the structure of a fibration of several metric factors over a base manifold $\Sigma$,

$$
d s^{2}=f_{1}^{2} d s_{M_{1}}^{2}+f_{2}^{2} d s_{M_{2}}^{2}+\cdots+f_{n}^{2} d s_{M_{n}}^{2}+d s_{\Sigma}^{2}
$$

The metric factors $f_{i}$ and $d s_{\Sigma}^{2}$, as well as the axion and dilaton fields are functions of $\Sigma$ only. The 3- and 5-form field strengths also depend on $\Sigma$; in view of their invariance under bosonic symmetries, they depend on $M_{i}$ only through the invariant volume forms on $M_{i}$.

The spaces $M_{i}$ are chosen to realize the bosonic symmetries of the half-BPS configuration which often leads to a product of lower dimensional $A d S$ and sphere factors. ${ }^{3}$ In view of the overall Minkowski signature of the full space-time metric $d s^{2}$, no more than a single Minkowski signature $A d S$ factor should occur. In some solutions, an extra factor of the real line $\mathbf{R}$ will be part of the space-time manifold. Thus, we may have,

$$
\begin{array}{ll}
M_{i}=A d S_{d+1} & \text { isometry : } S O(2, d) \\
M_{i}=S^{d} & \text { isometry : } S O(d+1) \\
M_{i}=\mathbf{R} & \text { isometry : } \mathbf{R}
\end{array}
$$

On the CFT side, the $S O(2, d)$ and $S O(d+1)$ isometries are associated respectively with the conformal symmetry, and the R-symmetry of the corresponding half-BPS operator.

A purely bosonic field configuration will preserve 16 supersymmetries provided there exist 16 linearly independent spinors $\varepsilon$ for which the supersymmetry transformations of the

\footnotetext{
${ }^{3}$ For some of the new solutions, which we shall advocate in Section 5 , the isometry algebra will have components of the form $S U(3), S U(1,2)$ and $S U(1,3)$, with corresponding space-time manifold factors $M_{i}$ of the form $\mathrm{CP}_{2}, \mathrm{CH}_{2}$, and $\mathrm{CH}_{3}$. This generalization of (2.7) will be discussed systematically in section 5.2 .
} 
fermion fields, namely the gravitino and the dilatino, vanish. Using the supersymmetry variations of these fermion fields [51], the condition leads to the following BPS equations,

$$
\begin{aligned}
0 & =i(\Gamma \cdot P) \varepsilon^{*}-\frac{i}{24}(\Gamma \cdot G) \varepsilon \\
0 & =D_{M} \varepsilon+\frac{i}{480}(\Gamma \cdot F) \Gamma_{M} \varepsilon-\frac{1}{96}\left(\Gamma_{M}(\Gamma \cdot G)+2(\Gamma \cdot G) \Gamma^{M}\right) \varepsilon^{*}
\end{aligned}
$$

where $P, G$, and $F$ stand for the Type IIB dilaton/axion, 3-form, and 5-form field strengths respectively. The unbroken supersymmetries are constructed using the invariant or Killing spinors on the spaces $M_{i}$. We will briefly review the known solutions associated with halfBPS operators in the next subsection. A unifying feature of all solutions is that the extremely complicated system of BPS equations arising from (2.9) can be solved exactly in terms of harmonic functions subject to certain prescribed boundary conditions.

\subsection{Known families of half-BPS solutions}

In this subsection, we shall summarize the known families of half-BPS solutions. In each case, the solutions are locally asymptotic to $A d S_{5} \times S^{5}$, and invariant under a subalgebra with 16 fermionic generators of $P S U(2,2 \mid 4)$. (The embeddings of Lie superalgebras will be discussed in detail in Section 4, and Appendices B and C.)

- The supergravity solutions of [5] correspond to the AdS dual of localized half-BPS operators on the CFT side. The solutions have $\mathbf{R} \oplus S O(4) \oplus S O(4)$ isometry and hence $M_{1}=S^{3}, M_{2}=S^{3}, M_{3}=\mathbf{R}$. The only non-trivial fields are the metric and the five form $F$, while the dilaton is constant, and the axion, and 3-form antisymmetric field strengths vanish. The 3-dimensional base space $\Sigma$ is constrained further by Killing spinors. The most general half-BPS solution with these symmetries is given by a 3-dimensional harmonic function $h$ defined on a 3-dimensional half space $\left(x_{1}, x_{2}, y\right)$ where $y>0$. The boundary condition $h= \pm \frac{1}{2}$ at $y=0$ completely determines the regular solutions. The symmetry preserved by these solutions is the superalgebra $\mathbf{R} \oplus P S U(2 \mid 2) \oplus P S U(2 \mid 2)$.

- The full supergravity solution corresponding to half-BPS Wilson loops was constructed exactly in [9]. (For an earlier derivation of the BPS equations and discussion of suitable boundary conditions, see $[52,53])$. The isometries are $S O(2,1) \oplus S O(3) \oplus S O(5)$ and hence we have $M_{1}=A d S_{2}, M_{2}=S^{2}$, and $M_{3}=S^{4}$. The 3-form field strength $G$ is, in general, non-vanishing. The base space $\Sigma$ is a 2-dimensional hyperelliptic Riemann surface with a boundary and the solution is completely determined by two harmonic functions with alternating Neumann and vanishing Dirichlet conditions on segments of the boundary. These 
solutions were analyzed further in $[54,55]$. The symmetry preserved by these solutions is the superalgebra $O S p\left(4^{*} \mid 4\right)$.

- The supergravity solutions dual to half-BPS surface operators were constructed in $[34,33]$ as double analytic continuations of the solutions of [5]. The isometries are $S O(2,2) \oplus$ $S O(4) \oplus U(1)$, and hence $M_{1}=A d S_{3}, M_{2}=S^{3}$, and $M_{3}=\mathbf{R}$. The base space $\Sigma$ is 3 dimensional in view of the $U(1)$ isometry. The symmetry preserved by these solutions is the superalgebra $P S U(1,1 \mid 2) \oplus P S U(1,1 \mid 2) \oplus U(1)$.

- The supergravity solutions corresponding to the planar half-BPS defect/interface were obtained exactly in $[7,8]$. (For an earlier derivation of the BPS equations and of suitable boundary conditions, see [56].) These solutions have isometry algebra $S O(2,3) \oplus S O(3) \oplus$ $S O(3)$, and hence we have $M_{1}=A d S_{4}, M_{2}=S^{2}$ and $M_{3}=S^{2}$. The 3-form field strength $G$ is, in general, non-vanishing. As in the case of the supergravity solutions dual to the Wilson loop, the base space $\Sigma$ is a hyperelliptic Riemann surface with boundary and the solution is determined by two harmonic functions with alternating Neumann and Dirichlet boundary conditions, although the detailed regularity conditions are different. The symmetry preserved by the defect/interface solutions is the superalgebra $O S p(4 \mid 4, \mathbf{R})$.

Whenever the CFT operator is not strictly speaking local, the corresponding AdS dual supergravity solutions are not globally asymptotic to $A d S_{5} \times S^{5}$, as the boundary of spacetime is deformed at the imprint of the operator on the boundary. This is the case for a Wilson line where the dual string ends at the boundary of $A d S_{5} \times S^{5}$, as well as for an interface where the boundary consists of multiple asymptotic regions of $A d S_{5} \times S^{5}$ glued together along the interface. In both cases, the solutions are asymptotically $A d S_{5} \times S^{5}$ away from the support of the operator. 


\section{Half-BPS configuration in M-theory}

In this section we shall generalize the analysis of Section 2 to M-theory. Eleven-dimensional supergravity admits two maximally supersymmetric Freund-Rubin solutions [1]. One is $A d S_{7} \times S^{4}$, which arises in the near-horizon limit of a stack of parallel $M 5$ branes; the other is $A d S_{4} \times S^{7}$, which arises in the near-horizon limit of a stack of parallel $M 2$ branes [24].

\subsection{The AdS/CFT correspondence for M-theory}

Two cases may be distinguished according to the two Freund-Rubin solutions. The first case provides a correspondence between 11-dimensional supergravity on $A d S_{7} \times S^{4}$ and the 5+1-dimensional CFT with 32 supersymmetries, which we shall denote by $\mathrm{CFT}_{6}$. The global symmetries here are encoded in the superalgebra $O S p\left(8^{*} \mid 4\right)$, whose maximal bosonic subalgebra is $S O\left(8^{*}\right) \oplus S p(4)=S O(2,6) \oplus S O(5)$. On the CFT side, $S O\left(8^{*}\right)=S O(2,6)$ is the $5+1$-dimensional conformal algebra, while $S O(5)$ is the R-symmetry. On the AdS side, $S O(2,6) \oplus S O(5)$ is the isometry of $A d S_{7} \times S^{4}$. The second case provides a correspondence between 11-dimensional supergravity on $A d S_{4} \times S^{7}$ and the 2+1-dimensional CFT with 32 supersymmetries, which we shall denote by $\mathrm{CFT}_{3}$. The global symmetries are encoded in the superalgebra $O S p(8 \mid 4, \mathbf{R})$, whose maximal bosonic subalgebra is $S O(8) \oplus S p(4, \mathbf{R})$. On the CFT side, $S p(4, \mathbf{R})=S O(2,3)$ is the $2+1$-dimensional conformal algebra, while $S O(8)$ is the R-symmetry. On the AdS side, $S O(8) \oplus S O(2,3)$ is the isometry of $A d S_{4} \times S^{7}$.

Although $\mathrm{CFT}_{6}$ and $\mathrm{CFT}_{3}$ are not as well understood as their 3+1-dimensional counterpart, many helpful facts analogous to the results derived in $\mathcal{N}=4$ SYM remain true. The unique $\mathrm{CFT}_{6}$ vacuum has maximal symmetry, given by the superalgebra $O S p\left(8^{*} \mid 4\right)$, while the unique $\mathrm{CFT}_{3}$ vacuum has symmetry $\operatorname{OSp}(8 \mid 4, \mathbf{R})$. Inserting an operator $\mathcal{O}$ in the CFT vacuum produces a new state whose symmetry is reduced to the subalgebra $\mathcal{H}$ of $O S p\left(8^{*} \mid 4\right)$ or $O S p(8 \mid 4, \mathbf{R})$ that leaves $\mathcal{O}$ invariant. The insertion of $\mathcal{O}$ on the CFT side causes the M-theory solution to deform away from $A d S_{7} \times S^{4}$ or $A d S_{4} \times S^{7}$. If the operator $\mathcal{O}$ is "sufficiently local", then there will exist an asymptotic bulk region in $\mathbf{R}^{6}$ or $\mathbf{R}^{3}$, away from the support of $\mathcal{O}$, where locally the state of the CFT is close to the vacuum state. The asymptotics at space-time infinity of the $A d S$ dual supergravity solution then coincide locally with $A d S_{7} \times S^{4}$ or $A d S_{4} \times S^{7}$.

This AdS/CFT argumentation shows that any half-BPS solution which is AdS/CFT dual to a "sufficiently local" operator in the CFT will obey the local asymptotics of $A d S_{7} \times S^{4}$ or $A d S_{4} \times S^{7}$, and will be invariant under a subalgebra $\mathcal{H}$ with 16 fermionic generators of respectively $O S p\left(8^{*} \mid 4\right)$ or $O S p(8 \mid 4, \mathbf{R})$. 


\subsection{Half-BPS operators in M-theory}

The $\mathrm{CFT}_{6}$ may be viewed as the decoupling limit of the $(0,2)$ worldvolume theory of a stack of parallel M5 branes, while the $\mathrm{CFT}_{3}$ may be defined as the IR fixed point of a $2+1$ dimensional supersymmetric $S U(N)$ SYM. In both cases, the definition of the CFT does not provide a direct Lagrangian description suitable to a weak coupling expansion of the quantum theory. This situation makes the analysis of the CFT more complicated than in the case of 3+1-dimensional $\mathcal{N}=4 \mathrm{SYM}$. (For recent progress on a Lagrangian formulation for multiple $M 2$-branes, see e.g. [57, 58, 59, 60]).

The local half-BPS operators of $\mathrm{CFT}_{6}$ were classified in [61]. For $\mathrm{CFT}_{6}$ on $\mathbf{R} \times S^{5}$, the chiral primary operators are time-translation invariant, do not depend on $S^{5}$, and preserve an $S O(3)$ subgroup of R-symmetry of the $(0,2)$ supersymmetry. The resulting bosonic symmetry associated with localized half-BPS operators is thus $\mathbf{R} \oplus S O(6) \oplus S O(3)$. As far as we know, a systematic analysis of non-local half-BPS operators in M-theory along the lines of the previous section has not been carried out.

\subsection{Intersecting branes}

The rules for supersymmetric intersections of M-branes are most easily derived by descending to 10-dimensional Type IIA superstring theory via compactification on a circle. The supersymmetric intersections of M5 and M2 branes preserving 8 of the 32 supersymmetries are listed in Table 3. A near-horizon limit may lead to an enhancement of the Poincaré and supersymmetry to superconformal symmetry on the intersection.

\begin{tabular}{|c|c|c|c|}
\hline Case & branes & dim intersec & bosonic symmetry \\
\hline \hline 1 & M5/M5 & 2 & $I S O(1,1) \oplus S O(4) \oplus S O(4)$ \\
\hline 2 & M5/M2 & 2 & $I S O(1,1) \oplus S O(4) \oplus S O(4)$ \\
\hline 3 & M5/M5 & 4 & $I S O(3,1) \oplus S O(3) \oplus S O(2)$ \\
\hline 4 & M2/M2 & 1 & $\mathbf{R} \oplus S O(6) \oplus S O(2) \oplus S O(2)$ \\
\hline
\end{tabular}

Table 3: Intersecting M2 and M5 branes with 8 supersymmetries

\subsection{Probe branes in $A d S_{7} \times S^{4}$ and $A d S_{4} \times S^{7}$}

Supersymmetric probe branes can be considered in the $A d S_{7} \times S^{4}$ near-horizon limit of $M 5$ branes and in the $A d S_{4} \times S^{7}$ near-horizon limit of $M 2$ branes. Just as in the case of $D p$ 
branes discussed in the previous section, one finds stable probe branes with world-volumes which are products of a lower dimensional $A d S$ space embedded into $A d S_{7} / A d S_{4}$ and a lower dimensional sphere embedded into $S^{4} / S^{7}$ respectively. The supersymmetry of the embedding follows from the existence of a $\kappa$-symmetry projector as in the Type IIB case. The supersymmetry of the probe brane was analyzed in [62,63].

Probe branes preserving 16 supersymmetries in $A d S_{7} \times S^{4}$, and in $A d S_{4} \times S^{7}$ backgrounds are listed respectively in Table 4, and Table 5.

\begin{tabular}{|c|c|c|c|}
\hline Case & probe & worldvolume $M$ & bosonic symmetry \\
\hline \hline 1 & $\mathrm{M} 5$ & $A d S_{3} \times S_{3}$ & $S O(2,2) \oplus S O(4) \oplus S O(4)$ \\
\hline 2 & $\mathrm{M} 2$ & $A d S_{3}$ & $S O(2,2) \oplus S O(4) \oplus S O(4)$ \\
\hline 3 & $\mathrm{M} 5$ & $A d S_{5} \times S^{1}$ & $S O(2,4) \oplus S O(3) \oplus S O(2)$ \\
\hline
\end{tabular}

Table 4: Probe branes in $A d S_{7} \times S^{4}$ preserving 16 supersymmetries

\begin{tabular}{|c|c|c|c|}
\hline Case & probe & worldvolume $M$ & bosonic symmetry \\
\hline \hline 4 & M2 & $A d S_{2} \times S^{1}$ & $S O(2,1) \oplus S O(6) \oplus S O(2)$ \\
\hline 5 & M5 & $A d S_{3} \times S^{3}$ & $S O(2,2) \oplus S O(4) \oplus S O(4)$ \\
\hline
\end{tabular}

Table 5: Probe branes in $A d S_{4} \times S^{7}$ preserving 16 supersymmetries

Case 2 of Table 4 , and case 5 of Table 5 may be viewed as different near-horizon limits of the M2/M5 intersecting brane configurations, and thus both correspond to case 2 of Table 3. The other cases of Tables 4 and 5 match the remaining cases in Table 3. Note that, for cases 3 and 4 , a supersymmetric embedding only preserves a single $S O(2)$ symmetry.

\subsection{Exact supergravity solutions}

Supergravity solutions to 11-dimensional supergravity are constructed following the same methods as were discussed in Section 2.5 for Type IIB supergravity. A purely bosonic field configuration will preserve 16 supersymmetries, provided there exist 16 linearly independent spinors $\varepsilon$ for which the supersymmetry variation of the gravitino field vanishes. Using the supersymmetry transformations of [64], the condition leads to the following BPS equation,

$$
\nabla_{M} \varepsilon+\frac{1}{288}\left(\Gamma_{M}{ }^{N P Q R}-8 \delta_{M}^{N} \Gamma^{P Q R}\right) F_{N P Q R} \varepsilon=0
$$


The metric is given by a fibration (2.7) over a base space $\Sigma$, and products of space $M_{i}$ respecting the expected symmetry of the solution. For M-theory, the total dimension of $M_{i}$ 's and $\Sigma$ is eleven. The 4 -form field strength $F_{M N P Q}$ can depend on $\Sigma$ and on the invariant volume forms of $M_{i}$.

\subsection{Known families of half-BPS solutions}

In this section, we shall summarize the known or partially known half-BPS solutions to 11-dimensional supergravity.

- Half-BPS solutions dual to local half-BPS operators were constructed in [5]. They have $S O(6) \oplus S O(3) \oplus \mathbf{R}$ isometry and thus we have $M_{1}=S^{5}, M_{2}=S^{2}, M_{3}=\mathbf{R}$. The remaining base manifold $\Sigma$ is 3-dimensional. In contrast to the Type IIB case, obtaining solutions here requires solving of a 3-dimensional Toda-like field equation with certain boundary conditions imposed for regularity. This equation has not been solved explicitly, so that solutions are only partially known. The solutions are asymptotically $A d S_{4} \times S^{7}$. They are invariant under the $S U(4 \mid 2)$ subalgebra of $O S p(8 \mid 4, \mathbf{R})$, which in turn leaves $A d S_{4} \times S^{7}$ invariant.

- Half BPS-solutions dual to a 1+1-dimensional planar defect/interface in $\mathrm{CFT}_{3}$, and to a 2-dimensional surface operator in $\mathrm{CFT}_{6}$ were constructed exactly in [10], at the local level. (For an earlier derivation of the BPS equations, and a partial solution, see [52, 63].) Regularity conditions, and global solutions are obtained in [65]. The isometry in both cases is given by $S O(2,2) \oplus S O(4) \oplus S O(4)$ and hence $M_{1}=A d S_{3}, M_{2}=M_{3}=S^{3}$. The base manifold $\Sigma$ is a 2-dimensional Riemann surface with boundary and the solution is completely specified by one harmonic function on $\Sigma$, and a certain integral transform of two further harmonic functions [10]. For the M5 brane these supergravity solutions are the dual description of the self-dual string solution of the M5 brane worldvolume theory found in [66].

For the 1+1-dimensional planar defect/interface supergravity solutions, the asymptotic behavior is locally $A d S_{4} \times S^{7}$, and the solutions are invariant under the $O S p(4 \mid 2, \mathbf{R}) \oplus$ $O S p(4 \mid 2, \mathbf{R})$ subalgebra of $O S p(8 \mid 4, \mathbf{R})$, which in turn leaves $A d S_{4} \times S^{7}$ invariant. For the 2-dimensional surface operator supergravity solutions, the asymptotic behavior is locally $A d S_{7} \times S^{4}$, and the solutions are invariant under the $O S p\left(4^{*} \mid 2\right) \oplus O S p\left(4^{*} \mid 2\right)$ subalgebra of $O S p\left(8^{*} \mid 4\right)$, which in turn leaves $A d S_{7} \times S^{4}$ invariant.

- Remarkably, the above families of half BPS-solutions on $A d S_{3} \times S^{3} \times S^{3}$ warped over a Riemann surface $\Sigma$ correspond to special points $c=1,-2,-1 / 2$ in a family of solutions parametrized by one real parameter $c$ [10]. The discussion of the asymptotics and superalgebra aspects of these general values of $c$ will be postponed until Section 7.2 below. 


\section{Lie superalgebras and their subalgebras}

A Lie superalgebra $\mathcal{G}$ is a $\mathbf{Z}_{2}$-graded associative algebra which admits a unique decomposition $\mathcal{G}=\mathcal{G}_{\overline{0}} \oplus \mathcal{G}_{\overline{1}}$ under the $\mathbf{Z}_{2}$-grading into an even (or bosonic) subspace $\mathcal{G}_{\overline{0}}$, and an odd (or fermionic) subspace $\mathcal{G}_{\overline{1}}$, and is equipped with a (graded) commutator,

$$
[A, B]=A B-(-1)^{\alpha \beta} B A
$$

which satisfies a generalized Jacobi-identity

$$
(-1)^{\alpha \gamma}[A,[B, C]]+(-1)^{\alpha \beta}[B,[C, A]]+(-1)^{\beta \gamma}[C,[A, B]]=0
$$

Here, $\alpha, \beta, \gamma$ are the $\mathbf{Z}_{2}$-gradings of $A, B, C \in \mathcal{G}$ respectively. A complete classification of simple Lie superalgebras was obtained in $[67,68,69,70]$. A dictionary of Lie superalgebras may be found in [71], which also contains an extensive bibliography on superalgebras.

Under the commutator operation, the bosonic subspace $\mathcal{G}_{\overline{0}}$ forms an ordinary Lie algebra, referred to as the maximal bosonic subalgebra. The fermionic subspace $\mathcal{G}_{\overline{1}}$ transforms under a representation of $\mathcal{G}_{\overline{0}}$, induced by the commutator. If $\mathcal{G}$ is simple, and the representation of $\mathcal{G}_{\overline{1}}$ under $\mathcal{G}_{\overline{0}}$ is completely reducible, then $\mathcal{G}$ is referred to as a classical Lie superalgebra. If $\mathcal{G}$ is classical and also has a non-degenerate invariant bilinear form, then $\mathcal{G}$ is referred to as a basic Lie superalgebra. In this paper, we shall restrict attention to the basic Lie superalgebras, since they are closest in properties to ordinary Lie algebras, and probably of greatest interest in physics. Their classification is also known [67, 68, 69, 70].

In this section, we will focus on the basic Lie superalgebras $P S U(2,2 \mid 4), \operatorname{OSp}\left(8^{*} \mid 4\right)$, and $O S p(8 \mid 4, \mathbf{R})$, of which we shall classify all basic Lie subalgebras (or direct sums thereof) which have 16 fermionic generators. In Section 6, we will turn our attention to conformal superalgebras, which are basic Lie superalgebras whose maximal bosonic subalgebra includes a conformal algebra in various dimensions. The above constitute the Lie superalgebras of greatest interest to AdS/CFT dualities. Since these facts will be needed throughout the paper, we shall begin by reviewing, in the next subsections, the classification of basic Lie superalgebras, their real forms, and their matrix representations.

\subsection{Basic Lie superalgebras over C}

We begin by listing, in Table 6 below, all basic superalgebras over $\mathbf{C}$, their maximal bosonic subalgebras, rank, and fermion representation content. 


\begin{tabular}{|c||c|c|c|c|}
\hline Superalgebra $\mathcal{G}$ & bosonic subalgebra $\mathcal{G}_{\overline{0}}$ & rank & fermion rep $\mathcal{G}_{\overline{1}}$ & $\#\left(\mathcal{G}_{\overline{1}}\right)$ \\
\hline \hline$S L(m \mid n), m \neq n$ & $S L(m) \oplus S L(n) \oplus \mathbf{C}$ & $m+n-1$ & $\left(m, n^{*}\right) \oplus\left(m^{*}, n\right)$ & $2 m n$ \\
\hline$P S L(m \mid m)$ & $S L(m) \oplus S L(m)$ & $2 m-2$ & $\left(m, m^{*}\right) \oplus\left(m^{*}, m\right)$ & $2 m^{2}$ \\
\hline$O S p(m \mid 2 n)$ & $S O(m) \oplus S p(2 n)$ & {$[m / 2]+n$} & $(m, 2 n)$ & $2 m n$ \\
\hline$G(3)$ & $S L(2) \oplus G_{2}$ & 3 & $(2,7)$ & 14 \\
\hline$F(4)$ & $S L(2) \oplus S O(7)$ & 4 & $\left(2,8_{s}\right)$ & 16 \\
\hline$D(2,1 ; c)$ & $S L(2) \oplus S L(2) \oplus S L(2)$ & 3 & $(2,2,2)$ & 8 \\
\hline
\end{tabular}

Table 6: Basic Lie superalgebras over C.

We have the following isomorphisms of basic Lie superalgebras over $\mathbf{C}$,

$$
\begin{aligned}
S L(m \mid n) & =S L(n \mid m) \\
O S p(2 \mid 2) & =S L(1 \mid 2) \\
O S p(4 \mid 2) & =D(2,1 ; 1) \\
D\left(2,1 ; c^{\prime}\right) & =D(2,1 ; c) \quad \quad c^{\prime} \in A(c)
\end{aligned}
$$

where the set $A(c)$ is defined by,

$$
A(c) \equiv\left\{c, 1 / c,-(1+c)^{ \pm 1},-(1+1 / c)^{ \pm 1}\right\}
$$

\subsection{Real forms}

In physics, and in particular in supergravity, superalgebras act on various fields, some of which are invariably real. This is the case of the space-time metric, and the antisymmetric 4 -form field in Type IIB. In M-theory, the 3-form field is real, and the gravitino is subject to a Majorana condition. Thus, we consider not just Lie superalgebras over $\mathbf{C}$, as in Table 6 , but we need also their various real forms, such as $P S U(2,2 \mid 4), O S p\left(8^{*} \mid 4\right)$, and $\operatorname{OSp}(8 \mid 4, \mathbf{R})$.

A real form of a Lie superalgebra $\mathcal{G}$ over $\mathbf{C}$ may be defined, and constructed, as follows (see for example $[72,71]$ ). Let $\phi$ be an involution from $\mathcal{G}$ to $\mathcal{G}$, which preserves the $\mathbf{Z}_{2}$-grading of $\mathcal{G}$, the graded commutator, and which is a semi-linear map,

$$
\begin{aligned}
\phi\left(\mathcal{G}_{\alpha}\right) & =\mathcal{G}_{\alpha} & & \alpha \in\{\overline{0}, \overline{1}\} \\
\phi([X, Y]) & =[\phi(X), \phi(Y)] & & X, Y \in \mathcal{G} \\
\phi(a X+b Y) & =\bar{a} \phi(X)+\bar{b} \phi(Y) & & a, b \in \mathbf{C}
\end{aligned}
$$

Here, $\bar{a}, \bar{b}$ stand for the standard complex conjugates of the complex numbers $a, b$. The last condition gives to $\phi$ the meaning of a generalized complex conjugation operation. Given 
such a $\phi$, the real Lie subalgebra $\mathcal{G}^{\phi}$ may be defined by,

$$
\mathcal{G}^{\phi} \equiv\{X+\phi(X), X \in \mathcal{G}\}
$$

It is readily checked that $\mathcal{G}^{\phi}$ is a Lie subalgebra of $\mathcal{G}$; it is real in the sense that $\phi\left(\mathcal{G}^{\phi}\right)=\mathcal{G}^{\phi}$. One refers to $\mathcal{G}^{\phi}$ as the real form of $\mathcal{G}$ associated with $\phi$.

The complete list of possible real forms of the basic Lie superalgebras is reviewed in Table 7 below (see $[67,72,71]$ ). For the case of the Lie superalgebras $S L(m \mid m)$, and their associated real forms, the $\mathbf{R}$ - or $U(1)$ - term is to be removed in the last column. For the definitions of the real forms $S U\left(2 m^{*}\right)$ and $S O\left(2 m^{*}\right)$, and isomorphisms between ordinary Lie algebras of low rank, see also Appendix A. Of special importance to us will be the real forms $P S U(2,2 \mid 4), O S p\left(8^{*} \mid 4\right)$, and $O S p(8 \mid 4, \mathbf{R})$, whose maximal bosonic subalgebras are respectively $S U(2,2) \oplus S U(4), S O(2,6) \oplus S p(4)$, and $S O(8) \oplus S p(4, \mathbf{R})$.

\begin{tabular}{|c||c|c|}
\hline Superalgebra $\mathcal{G}$ & real form $\mathcal{G}^{\phi}$ & maximal bosonic subalgebra $\mathcal{G}_{\overline{0}}^{\phi}$ \\
\hline \hline$S L(m \mid n)$ & $S L(m \mid n, \mathbf{R})$ & $S L(m, \mathbf{R}) \oplus S L(n, \mathbf{R}) \oplus \mathbf{R}$ \\
$S L\left(m+m^{\prime} \mid n+n^{\prime}\right)$ & $S U\left(m, m^{\prime} \mid n, n^{\prime}\right)$ & $S U\left(m, m^{\prime}\right) \oplus S U\left(n, n^{\prime}\right) \oplus U(1)$ \\
$S L(2 m \mid 2 n)$ & $S L(2 m \mid 2 n, \mathbf{H})$ & $S U\left(2 m^{*}\right) \oplus S U\left(2 n^{*}\right) \oplus \mathbf{R}$ \\
\hline$O S p\left(m+m^{\prime} \mid 2 n\right)$ & $O S p\left(m, m^{\prime} \mid 2 n, \mathbf{R}\right)$ & $S O\left(m, m^{\prime}\right) \oplus S p(2 n, \mathbf{R})$ \\
$O S p\left(2 m \mid 2 n+2 n^{\prime}\right)$ & $O S p\left(2 m^{*} \mid 2 n, 2 n^{\prime}\right)$ & $S O\left(2 m^{*}\right) \oplus S p\left(2 n, 2 n^{\prime}\right)$ \\
\hline$F(4)$ & $F(4 ; p), p=0,1,2,3$ & $S L(2, \mathbf{R}) \oplus S O(p, 7-p)$ \\
\hline$G(3)$ & $G(3 ; p), p=0,1$ & $S L(2, \mathbf{R}) \oplus G_{2,2 p}$ \\
\hline$D(2,1 ; c)$ & $D(2,1 ; c ; p), p=0,1,2$ & $S L(2, \mathbf{R}) \oplus S O(p, 4-p)$ \\
\hline
\end{tabular}

Table 7: Real forms of the basic Lie superalgebras.

We have the following isomorphisms of real forms of low rank,

$$
\begin{array}{rlr}
O S p\left(2^{*} \mid 2\right) & =S U(2 \mid 1) & \\
O S p(2 \mid 2, \mathbf{R}) & =S U(1,1 \mid 1) & \\
D(2,1 ; 1 ; 0) & =O S p(4 \mid 2, \mathbf{R}) & \\
D(2,1 ; c ; 0) & =O S p\left(4^{*} \mid 2\right) & \\
D(2,1 ; 1 ; 1) & =O S p(1,3 \mid 2, \mathbf{R}) & \\
D(2,1 ; 1 ; 2) & =O S p(2,2 \mid 2, \mathbf{R}) & \\
D(2,1 ; c ; 0) & =D(2,1 ; 1 / c ; 0) & \\
D(2,1 ; c ; 2) & =D\left(2,1 ; c^{\prime} ; 2\right) & \text { for } c=-2,-1 / 2
\end{array}
$$

as well as $S U(n \mid m)=S U(m \mid n)$, and $S L(m \mid n, \mathbf{R})=S L(n \mid m, \mathbf{R})$ for all $m, n$. 


\subsection{Matrix representation of the $S L, S U$ and $O S p$ series}

The Lie superalgebras in the $S L, S U$, and $O S p$ series may be defined in terms of matrix representations, which are especially useful in defining the real forms of these algebras. Let $\mathcal{V}=\mathcal{V}_{\overline{0}} \oplus \mathcal{V}_{\overline{1}}$ be a $\mathbf{Z}_{2}$-graded vector space, where $\operatorname{dim}\left(\mathcal{V}_{\overline{0}}\right)=m$ and $\operatorname{dim}\left(\mathcal{V}_{\overline{1}}\right)=n$. A supermatrix $M$ is a linear map of $\mathcal{V}$ into $\mathcal{V}$ which may be decomposed as follows,

$$
M\left(\begin{array}{l}
\mathcal{V}_{\overline{0}} \\
\mathcal{V}_{\overline{1}}
\end{array}\right) \rightarrow\left(\begin{array}{l}
\mathcal{V}_{\overline{0}} \\
\mathcal{V}_{\overline{1}}
\end{array}\right) \quad M=\left(\begin{array}{cc}
A & B \\
C & D
\end{array}\right)
$$

where $A, D$ are even grading and of dimension $m \times m$ and $n \times n$ respectively, and where $B, C$ are odd grading and of dimension $m \times n$ and $n \times m$ respectively. The general linear Lie superalgebra $G L(m \mid n)$ is defined to be the set of all such matrices over $\mathbf{C}$. The supertrace is defined by $\operatorname{str}(M) \equiv \operatorname{tr}(A)-\operatorname{tr}(D)$, and the supertranspose is defined by

$$
M^{s t} \equiv\left(\begin{array}{cc}
A^{t} & C^{t} \\
-B^{t} & D^{t}
\end{array}\right) \quad\left(M^{s t}\right)^{s t}=\left(\begin{array}{cc}
A & -B \\
-C & D
\end{array}\right)
$$

Note that the double supertranspose takes the value $(-1)^{\alpha}$ on $\mathcal{V}_{\alpha}$. The matrix representation of $S L(m \mid n)$ and $O S p(m \mid 2 n)$ over $\mathbf{C}$ are given by,

$$
\begin{aligned}
S L(m \mid n) & =\{M \in G L(m \mid n), \operatorname{str}(M)=0\} \\
O S p(m \mid 2 n) & =\left\{M \in G L(m \mid 2 n), M^{s t} K_{m \mid 2 n}+K_{m \mid 2 n} M=0\right\}
\end{aligned}
$$

Here, the matrix $K_{m \mid 2 n}$ is defined as follows,

$$
K_{m \mid 2 n} \equiv\left(\begin{array}{cc}
I_{m} & 0 \\
0 & J_{2 n}
\end{array}\right) \quad J_{2 n} \equiv\left(\begin{array}{cc}
0 & I_{n} \\
-I_{n} & 0
\end{array}\right)
$$

where $I_{m}$ is the identity matrix in dimension $m$. Note that the expression for $K_{m \mid 2 n}$ in the definition of $O S p(m \mid 2 n)$ is consistent with the fact that the double supertranspose reverses the sign of the odd parts $B, C$. It is readily shown that the matrix realization given here is equivalent to the definition given in [67].

The matrix representations of the real forms may be obtained in a similar way. To do so, we define the following matrices,

$$
\begin{array}{rlrl}
L_{m, m^{\prime} \mid n, n^{\prime}} & \equiv\left(\begin{array}{cc}
I_{m, m^{\prime}} & 0 \\
0 & -i I_{n, n^{\prime}}
\end{array}\right) & I_{m, m^{\prime}} \equiv\left(\begin{array}{cc}
I_{m} & 0 \\
0 & -I_{m^{\prime}}
\end{array}\right) \\
K_{m, m^{\prime} \mid 2 n} \equiv\left(\begin{array}{cc}
I_{m, m^{\prime}} & 0 \\
0 & J_{2 n}
\end{array}\right) & \tilde{K}_{2 m \mid 2 n, 2 n^{\prime}} \equiv\left(\begin{array}{cc}
J_{2 m} & 0 \\
0 & I_{2 n, 2 n^{\prime}}
\end{array}\right)
\end{array}
$$


The matrix representation of real forms is given in terms of the semi-linear involutions $\phi$ by ${ }^{4}$

$$
\begin{aligned}
S L(m \mid n ; \mathbf{R}) & =\left\{M \in S L(m \mid n), M=\phi(M) ; \phi(M) \equiv M^{*}\right\} \\
S U\left(m, m^{\prime} \mid n, n^{\prime}\right) & =\left\{M \in S L\left(m+m^{\prime} \mid n+n^{\prime}\right), M=\phi(M) ; \phi(M) \equiv-L^{-1}\left(M^{*}\right)^{s t} L\right\} \\
O S p\left(2 m^{*} \mid 2 n, 2 n^{\prime}\right) & =\left\{M \in O S p\left(2 m \mid 2 n+2 n^{\prime}\right), M=\phi(M) ; \phi(M) \equiv-\tilde{K}^{-1}\left(M^{*}\right)^{s t} \tilde{K}\right\} \\
O S p\left(m, m^{\prime} \mid 2 n, \mathbf{R}\right) & =\left\{M \in S L\left(m+m^{\prime} \mid 2 n ; \mathbf{R}\right), M=-K^{-1} M^{s t} K\right\}
\end{aligned}
$$

where we have used the abbreviations $L=L_{m, m^{\prime} \mid n, n^{\prime}}, \tilde{K}=\tilde{K}_{2 m \mid 2 n, 2 n^{\prime}}$, and $K=K_{m, m^{\prime} \mid 2 n}$. The matrix representations of the real forms $S U$ and $O S p$ given here are equivalent to the matrix formulations of [67].

\subsection{Subalgebras of the complex Lie superalgebras $S L$ and $O S p$}

A systematic classification of all Lie subalgebras, for a given basic Lie superalgebra, does not appear to be available. Therefore, we shall have to obtain the classification of all Lie subalgebras of $P S U(2,2 \mid 4), O S p\left(8^{*} \mid 4\right)$, and $O S p(8 \mid 4, \mathbf{R})$ with 16 fermionic generators by drawing together the known results and deriving here the remaining cases ourselves. From the outset, we shall restrict to considering only Lie subalgebras which are direct sums of basic Lie superalgebras and possibly ordinary simple Lie algebras. These appear to be the only cases that will be needed for AdS/CFT applications.

\begin{tabular}{|c||c|}
\hline Superalgebra & subalgebra \\
\hline \hline$S L\left(m+m^{\prime} \mid n+n^{\prime}\right)$ & $S L(m \mid n) \oplus S L\left(m^{\prime} \mid n^{\prime}\right) \oplus \mathbf{C}$ \\
\hline$O S p\left(m+m^{\prime} \mid 2 n+2 n^{\prime}\right)$ & $O S p(m \mid 2 n) \oplus O S p\left(m^{\prime} \mid 2 n^{\prime}\right)$ \\
\hline$S L(m \mid 2 n)$ & $O S p(m \mid 2 n)$ \\
\hline$O S p(2 m \mid 2 n)$ & $S L(m \mid n) \oplus \mathbf{C}$ \\
\hline
\end{tabular}

Table 8: Maximal subalgebras of the $S L$ and $O S p$ series over $\mathbf{C}$.

The known cases consist of the maximal regular subalgebras (for which the rank of the subalgebra coincides with the rank of the original algebra), and those singular subalgebras that can be obtained by folding the root diagram, both cases being considered as Lie superalgebras over $\mathbf{C}$. (The distinction between regular and singular subalgebras has to do

\footnotetext{
${ }^{4}$ To exhibit the involution $\phi$ more clearly on the last line, we may use the following alternative definition, $O S p\left(m, m^{\prime} \mid 2 n, \mathbf{R}\right)=\left\{M \in O S p\left(m+m^{\prime} \mid 2 n\right), M=\phi(M) ; \phi(M) \equiv I_{m, m^{\prime} \mid 2 n} M^{*} I_{m, m^{\prime} \mid 2 n}\right\}$. The two definitions are related by a (complex) change of basis, given by the square root of $I_{m, m^{\prime} \mid 2 n}$.
} 
with the root structure of the subalgebra [71], and will not be needed here.) The results for subalgebras of the $S L$ and $O S p$ series (subalgebras of the exceptional cases will not be needed here) are listed in Table 8 above. This list includes the cases where $m^{\prime} n^{\prime}=0$ by setting $S L(m \mid 0)=S L(m), O S p(m \mid 0)=S O(m)$, and $O S p(0 \mid 2 n)=S p(2 n)$. Using the matrix representations of the Lie superalgebras given in Section 4.2, it is straightforward to check that the embeddings of Table 8 indeed hold.

\subsection{Subalgebras of the real form Lie superalgebras $S U$ and $O S p$}

From the results on Lie subalgebras over $\mathbf{C}$, as well as from the matrix representations for the real forms of the superalgebras, given in Section 4.2, certain subalgebras of the real forms in the $S U$ and $O S p$ series may be deduced. The derivation of some of these results will be given in Appendix B. To illustrate here the key issue in the construction of real form subalgebras, we shall begin by deriving the following general result.

Let $\mathcal{H}$ be a subalgebra of the Lie superalgebra $\mathcal{G}$ defined as the invariance subalgebra of a (bosonic) element $T \in \mathcal{G}$,

$$
\mathcal{H}=\{M \in \mathcal{G},[T, M]=0\}
$$

Let $\phi$ be a semi-linear involution of $\mathcal{G}$ which preserves its $\mathbf{Z}_{2}$ grading, and graded commutator. The complete definition of $\phi$ was given in (4.5). If there exists a $\lambda \in \mathbf{C}$, such that

$$
\phi(T)=\lambda T
$$

then the semi-linearity and involutive properties of $\phi$ imply that $|\lambda|=1$. Any $\phi$ satisfying (4.15) produces a unique real form $\mathcal{H}^{\phi}$ of $\mathcal{H}$ which is a subalgebra of the real form $\mathcal{G}^{\phi}$, and

$$
\mathcal{H}^{\phi}=\mathcal{G}^{\phi} \cap \mathcal{H}
$$

The proof is immediate upon use of $\phi([T, M])=[\phi(T), \phi(M)]=\lambda[T, \phi(M)]$, for any $M \in \mathcal{G}$.

It is important to realize that a given real form $\mathcal{G}^{\phi}$ of a complex Lie superalgebra $\mathcal{G}$ may induce several different real forms of a given complex subalgebra $\mathcal{H} \subset \mathcal{G}$, provided $\mathcal{H}$ has several different embeddings into $\mathcal{G}$. When two different embeddings $\mathcal{H}_{(1)}$, and $\mathcal{H}_{(2)}$ of $\mathcal{H}$ into $\mathcal{G}$ are equivalent to one another, namely $\mathcal{H}_{(2)}=S \mathcal{H}_{(1)} S^{-1}$ for some $S$ in the maximal bosonic subgroup of $\mathcal{G}$, the real forms $\mathcal{H}_{(1)}^{\phi}$ and $\mathcal{H}_{(2)}^{\phi}$ will be isomorphic provided $\phi(S)=\lambda S$. If this relation does not hold, however, the two real forms $\mathcal{H}_{(1)}^{\phi}$ and $\mathcal{H}_{(2)}^{\phi}$ will generally be inequivalent, even though $\mathcal{H}_{(1)}$ and $\mathcal{H}_{(2)}$ are equivalent over $\mathbf{C}$. 
Henceforth, we shall restrict to the series of real forms needed in this paper, namely $S U\left(m, m^{\prime} \mid n\right), O S p(m \mid 2 n, \mathbf{R})$, and $O S p\left(2 m^{*} \mid 2 n\right)$. They are given in Table 9 below. The results on the first three lines of Table 9 are immediate consequences of the matrix representations for these superalgebras given in (4.13), and are available from [71]. The results on the fourth and fifth lines have been known to hold, when $p q=0$, for some time in the context of the oscillator realization of superalgebras $[73,74,75]$. As we are not aware of complete proofs elsewhere, these results will be proven generally in Appendix B, together with those of the last four lines of Table 9, for the sake of completeness.

\begin{tabular}{|c||c|}
\hline Real form superalgebra & real form subalgebra \\
\hline \hline$S U\left(p+p^{\prime}, q+q^{\prime} \mid n+n^{\prime}\right)$ & $S U(p, q \mid n) \oplus S U\left(p^{\prime}, q^{\prime} \mid n^{\prime}\right) \oplus U(1)$ \\
\hline$O S p\left(m+m^{\prime} \mid 2 n+2 n^{\prime}, \mathbf{R}\right)$ & $O S p(m \mid 2 n, \mathbf{R}) \oplus O S p\left(m^{\prime} \mid 2 n^{\prime}, \mathbf{R}\right)$ \\
\hline$O S p\left(2 m+2 m^{\prime *} \mid 2 n+2 n^{\prime}\right)$ & $O S p\left(2 m^{*} \mid 2 n\right) \oplus O S p\left(2 m^{\prime *} \mid 2 n^{\prime}\right)$ \\
\hline$O S p\left(2 m^{*} \mid 2 n\right)$ & $S U(p, q \mid n) \oplus U(1), p+q=m ; p, q \geq 0$ \\
\hline$O S p(2 m \mid 2 n, \mathbf{R})$ & $S U(m \mid p, q) \oplus U(1), p+q=n ; p, q \geq 0$ \\
\hline$S U(m \mid n, n)$ & $O S p(m \mid 2 n, \mathbf{R})$ \\
\hline$S U(m, m \mid 2 n)$ & $O S p\left(2 m^{*} \mid 2 n\right)$ \\
\hline
\end{tabular}

Table 9: Maximal subalgebras of relevant real forms of the $S U$ and $O S p$ series.

In addition, it will be proven in Appendix $\mathrm{C}$ that none of the real forms of the exceptional basic Lie superalgberas $F(4), G(3)$, and $D(2,1 ; c)$ for $c \neq 1,-2,-1 / 2$, is a subalgebra of $P S U(2,2 \mid 4), O S p\left(8^{*} \mid 4\right)$, and $O S p(8 \mid 4, \mathbf{R})$.

\subsection{Basic subalgebras with 16 fermionic generators}

All basic superalgebras over $\mathbf{C}$ (which are by definition simple) with 16 fermionic generators are readily identified from Table 6; they are $S L(4 \mid 2), S L(8 \mid 1), O S p(8 \mid 2), O S p(4 \mid 4), O S p(2 \mid 8)$, and $F(4)$. The algebras $S L(8 \mid 1)$ and $O S p(2 \mid 8)$ are manifestly not subalgebras of either $P S L(4 \mid 4)$, or $O S p(8 \mid 4)$, since their bosonic subalgebras do not fit. Also, the $S O(8)$ part of the maximal bosonic subalgebra of $O S p(8 \mid 2)$ cannot fit into $P S L(4 \mid 4)$, so that $O S p(8 \mid 2) \not \subset$ $P S L(4 \mid 4)$. In Appendix $\mathrm{C}$ it is shown that $F(4)$ is not a subalgebra of either $P S L(4 \mid 4)$, or $O S p(8 \mid 4)$. All remaining basic subalgebras over $\mathbf{C}$ are given by,

$$
\begin{array}{rll}
P S L(4 \mid 4) & \supset & S L(4 \mid 2), O S p(4 \mid 4) \\
O S p(8 \mid 4) & \supset & O S p(8 \mid 2), O S p(4 \mid 4), S L(4 \mid 2)
\end{array}
$$


It follows from Table 8 that these embedding relations over $\mathbf{C}$ hold in all cases of (4.17). Note that some of these embeddings are not maximal and admit purely bosonic Lie algebra enhancements in the cases $P S L(4 \mid 4) \supset S L(4 \mid 2) \oplus S L(2)$, and $O S p(8 \mid 4) \supset O S p(8 \mid 2) \oplus S p(2)$, and $O S p(8 \mid 4) \supset O S p(4 \mid 4) \oplus S O(4)$.

Next, we shall list the associated real forms. Several of these simple subalgebras are not in fact maximal subalgebras. In those cases, there exists a complementary purely bosonic subalgebra, which we shall list below in its maximal form. ${ }^{5}$

$$
\begin{aligned}
P S U(2,2 \mid 4) \supset \quad & S U(2,2 \mid 2) \oplus S U(2), S U(2 \mid 4) \oplus S U(2), \\
& S U(1,1 \mid 4) \oplus S U(1,1), \\
& O S p\left(4^{*} \mid 4\right), O S p(4 \mid 4, \mathbf{R}) \\
O S p\left(8^{*} \mid 4\right) \supset \quad & O S p\left(8^{*} \mid 2\right) \oplus S p(2), \\
& O S p\left(4^{*} \mid 4\right) \oplus S O\left(4^{*}\right), \\
& S U(4 \mid 2) \oplus U(1) \\
& S U(1,3 \mid 2) \oplus U(1) \\
& S U(2,2 \mid 2) \oplus U(1) \\
O S p(8 \mid 4, \mathbf{R}) \quad \supset \quad & O S p(8 \mid 2, \mathbf{R}) \oplus S p(2, \mathbf{R}) \\
& O S p(4 \mid 4, \mathbf{R}) \oplus S O(4), \\
& S U(4 \mid 2) \oplus U(1) \\
& S U(4 \mid 1,1) \oplus U(1)
\end{aligned}
$$

The existence of these embeddings follows immediately from the results of Table 9. The fact that no other real forms of the complex algebras are subalgebras may be shown by systematically considering all such possible real forms. The following cases may be easily excluded, by checking that the maximal bosonic subalgebras do not fit,

$$
\begin{array}{rll}
P S U(2,2 \mid 4) \quad \not \supset \quad & S U(1,3 \mid 2), S U(1,3 \mid 1,1), S U(2,2 \mid 1,1), \\
& O S p\left(4^{*} \mid 2,2\right), O S p(p, 4-p \mid 4, \mathbf{R}), p=1,2 \\
O S p\left(8^{*} \mid 4\right) \not \supset \quad & O S p(8 \mid 2, \mathbf{R}), O S p(p, 4-p \mid 4, \mathbf{R}), p=0,1,2, \\
& S U(2,2 \mid 1,1), S U(1,3 \mid 1,1) \\
O S p(8 \mid 4, \mathbf{R}) \not \supset & O S p\left(8^{*} \mid 2\right), O S p\left(4^{*} \mid 4\right), O S p(p, 8-p \mid 2, \mathbf{R}), p=1, \cdots, 4, \\
& S U(2,2 \mid 2), S U(1,3 \mid 2), S U(2,2 \mid 1,1), S U(1,3 \mid 1,1)
\end{array}
$$

\footnotetext{
${ }^{5}$ In the first version of this paper, the inclusions of $S U(1,3 \mid 2), S U(2,2 \mid 2)$, and $S U(1,1 \mid 4)$ were listed incorrectly; their correct inclusion will lead to cases IIIb, IIIc, and VIc in section 5.5. We are grateful to Jaume Gomis for questions that led us to find and correct these omissions.
} 


\subsection{Direct sum subalgebras with 16 fermionic generators over $\mathrm{C}$}

Subalgebras with two simple Lie superalgebra components or more offer further possibilities. We begin by presenting these subalgebras over $\mathbf{C}$. We list all simple basic superalgebras with strictly fewer than 16 fermionic generators (indicated in the left column below), and whose maximal bosonic subalgebra fits into either $P S L(4 \mid 4)$ or $O S p(8 \mid 4)$. They are. ${ }^{6}$

$\begin{array}{rl}2 & O S p(1 \mid 2) \\ 4 & S L(2 \mid 1)=\operatorname{OSp}(2 \mid 2), \operatorname{OSp}(1 \mid 4) \\ 6 & S L(3 \mid 1), \operatorname{OSp}(3 \mid 2) \\ 8 & P S L(2 \mid 2), \operatorname{OSp}(4 \mid 2), \operatorname{OSp}(2 \mid 4), S L(4 \mid 1), D(2,1 ; c) \\ 10 & O S p(5 \mid 2) \\ 12 & S L(3 \mid 2), \operatorname{OSp}(3 \mid 4), O S p(6 \mid 2) \\ 14 & O S p(7 \mid 2), G(3)\end{array}$

In Appendix $\mathrm{C}$, it will be proven that the exceptional superalgebras $G(3)$, and $D(2,1 ; c)$ with $c \neq 1,-2,-1 / 2$, are not subalgebras of $P S L(4 \mid 4)$ or $O S p(8 \mid 4)$.

To classify the non-exceptional cases, we shall make use of the subalgebra embeddings given in Table 8. From the table, we deduce that no direct sum involving $S L(4 \mid 1)$ can fit either in $P S L(4 \mid 4)$ or $O S p(8 \mid 4)$, because the $S L(4)$ component of $S L(4 \mid 1)$ is already maximal both in $P S L(4 \mid 4)$ and in $O S p(8 \mid 4)$. The same arguments lead us to eliminate the possibility of having $O S p(1 \mid 4), O S p(2 \mid 4)$ and $O S p(3 \mid 4)$ as subalgebras. The above considerations reduce the number of possible subalgebra components to the following list,

$\begin{array}{rl}2 & O S p(1 \mid 2) \\ 4 & S L(2 \mid 1)=O S p(2 \mid 2) \\ 6 & S L(3 \mid 1), O S p(3 \mid 2) \\ 8 & P S L(2 \mid 2), O S p(4 \mid 2) \\ 10 & O S p(5 \mid 2) \\ 12 & S L(3 \mid 2), O S p(6 \mid 2) \\ 14 & O S p(7 \mid 2)\end{array}$

\footnotetext{
${ }^{6}$ The Lie superalgebras $O S p(1 \mid 6), \operatorname{OSp}(1 \mid 8), \operatorname{OSp}(1 \mid 10), \operatorname{OSp}(1 \mid 12), \operatorname{OSp}(1 \mid 14), \operatorname{OSp}(2 \mid 6), S L(5 \mid 1)$, $S L(6 \mid 1)$, and $S L(7 \mid 1)$ also have fewer than 16 fermionic generators, but they manifestly do not fit into either $P S L(4 \mid 4)$ or $O S p(8 \mid 4)$. Thus, they are excluded from the list.
} 
The possible subalgebra components may be enumerated by ensuring that the total number of fermionic generators equals 16, and that the maximal bosonic subalgebras fit. As was the case with the basic subalgebras, an extra bosonic subalgebra may be allowed. In the table below, we shall include the maximal version of such a bosonic subalgebra, whenever it is allowed. Lie superalgebras with two or more components can only have $\mathbf{C}, U(1)$, or $\mathbf{R}$ as bosonic complements.

The resulting subalgebra embeddings are as follows,

$$
\begin{aligned}
P S L(4 \mid 4) \supset & P S L(2 \mid 2) \oplus P S L(2 \mid 2) \oplus \mathbf{C}^{2}, \\
& S U(2 \mid 3) \oplus S U(2 \mid 1) \\
O S p(8 \mid 4) \supset \quad & O S p(4 \mid 2) \oplus O S p(4 \mid 2), \\
& O S p(5 \mid 2) \oplus O S p(3 \mid 2), \\
& O S p(6 \mid 2) \oplus O S p(2 \mid 2) \\
& O S p(7 \mid 2) \oplus O S p(1 \mid 2),
\end{aligned}
$$

The non-trivial cases are eliminated as follows.

1. $S L(3 \mid 2) \oplus O S p(2 \mid 2)$ is not a subalgebra of $O S p(8 \mid 4)$. Note that both $O S p(8 \mid 4)$ and $S L(3 \mid 2) \oplus O S p(2 \mid 2)$ are of rank 6 . If the embedding existed, it would have to be a maximal regular subalgebra, but all such sub-algebras of $O S p(8 \mid 4)$ are known, and $S L(3 \mid 2) \oplus O S p(2 \mid 2)$ is not amongst them. (For example, $O S p(6 \mid 2) \oplus O S p(2 \mid 2)$ is a regular maximal subalgebra of $O S p(8 \mid 4)$, but $S L(3 \mid 2)$ does not fit into $O S p(6 \mid 2)$.) Finally, since the ranks of $S L(3 \mid 2) \oplus O S p(2 \mid 2)$ and $O S p(8 \mid 4)$ are equal, the embedding cannot be that of a non-regular subalgebra either.

2. $P S L(2 \mid 2) \oplus O S p(4 \mid 2)$ is not a subalgebra of $O S p(8 \mid 4)$. Note that the rank of $P S L(2 \mid 2)$ is only 2, so the total rank of $P S L(2 \mid 2) \oplus O S p(4 \mid 2)$ is 5 . This case is ruled out as follows. While the maximal bosonic subalgebra $S O(4) \oplus S p(2) \oplus S L(2) \oplus S L(2)$ fits, the fermionic generators in $P S L(2 \mid 2)$ transform in the spin $1 / 2$ representation of the $S L(2)$ components. But the first $S L(2)$ component is embedded into $S O(8)$ by the spin 1 representation of that same algebra, so that the fermionic generators do not fit.

Finally, subalgebras with three simple components or more are all ruled out by analogous arguments. Thus, the cases of (4.22) exhaust all possible subalgebras. 


\subsection{Direct sum subalgebras with 16 real fermionic generators}

The associated real forms are as follows,

$$
\begin{aligned}
P S U(2,2 \mid 4) \supset \quad & P S U(2 \mid 2) \oplus P S U(2 \mid 2) \oplus \mathbf{R}^{2}, \\
& P S U(1,1 \mid 2) \oplus P S U(1,1 \mid 2) \oplus U(1)^{2}, \\
& S U(2 \mid 3) \oplus S U(2 \mid 1), \\
& S U(1,1 \mid 3) \oplus S U(1,1 \mid 1), \\
& S U(1,2 \mid 2) \oplus S U(1 \mid 2) \\
O S p\left(8^{*} \mid 4\right) \supset \quad & O S p\left(4^{*} \mid 2\right) \oplus O S p\left(4^{*} \mid 2\right), \\
& O S p\left(2^{*} \mid 2\right) \oplus O S p\left(6^{*} \mid 2\right) \\
O S p(8 \mid 4, \mathbf{R}) \supset \quad & O S p(7 \mid 2, \mathbf{R}) \oplus O S p(1 \mid 2, \mathbf{R}), \\
& O S p(6 \mid 2, \mathbf{R}) \oplus O S p(2 \mid 2, \mathbf{R}), \\
& O S p(5 \mid 2, \mathbf{R}) \oplus O S p(3 \mid 2, \mathbf{R}), \\
& O S p(4 \mid 2, \mathbf{R}) \oplus O S p(4 \mid 2, \mathbf{R})
\end{aligned}
$$

Note that $O S p\left(2^{*} \mid 2\right) \oplus O S p\left(6^{*} \mid 2\right)$ is a subalgebra of $O S p\left(8^{*} \mid 4\right)$. The orthogonal parts of the bosonic subalgebras are respectively given by $S O\left(2^{*}\right) \oplus S O\left(6^{*}\right)=S O(2) \oplus S U(1,3)$ and $S O(2,6)$. The subalgebra $S U(1,3)$ is maximal in $S O(2,6)$, and the generator commuting with $S U(1,3)$ inside $S O(2,6)$ is indeed a compact $S O(2)$, whence the result.

\subsection{Enhanced supersymmetries}

Finally, we note that in various cases, there exist subalgebras $\mathcal{H}^{*}$ of the superalgebras $P S U(2,2 \mid 4), O S p\left(8^{*} \mid 4\right)$, or $O S p(8 \mid 4, \mathbf{R})$ whose number of fermionic generators actually exceeds 16 , but remains less than 32 (or even less than 28). If $\mathcal{H}^{*}$ contains one of the subalgebras $\mathcal{H}$ with precisely 16 fermionic generators that were classified in this section, then it may play the role of a symmetry algebra for half-BPS solutions with an enhanced supersymmetry. The systematic classification of such superalgebras $\mathcal{H}^{*}$ will be carried out in Section 7.1 , where their significance to the existence of half-BPS solutions with enhanced supersymmetry will be discussed as well. 


\section{Half-BPS supergravity solutions and superalgebras}

In the present section, we shall spell out and prove generally the correspondence between superalgebras $\mathcal{H}$ and the existence of half-BPS solutions. The classification of superalgebras $\mathcal{H}$ with 16 fermionic generators which are subalgebras of $P S U(2,2 \mid 4), O S p\left(8^{*} \mid 4\right)$, and $O S p(8 \mid 4, \mathbf{R})$, obtained in Section 4, allows us then to derive a classification of all possible families of half-BPS solutions which are locally asymptotic to respectively $A d S_{5} \times S^{5}$, $A d S_{7} \times S^{4}$, and $A d S_{4} \times S^{7}$ for each associated supergravity.

All known families of half-BPS solutions may be identified in this classification. The remaining cases, where solutions are not known at this time, will be discussed, and arguments for either their existence or their absence will be developed using input from the AdS/CFT correspondence and from intersecting brane and probe brane dynamics.

\subsection{The superalgebra - half-BPS solution correspondence}

The precise statement of the correspondence is as follows.

If a half-BPS solution of Type IIB is locally asymptotic to $A d S_{5} \times S^{5}$, or a half-BPS solution of $M$-theory is locally asymptotic to $A d S_{7} \times S^{4}$ or $A d S_{4} \times S^{7}$, and invariant under a superalgebra $\mathcal{H}$ (with 16 fermionic generators since the solution is assumed to be half-BPS), then $\mathcal{H}$ must be a subalgebra of $P S U(2,2 \mid 4), O S p\left(8^{*} \mid 4\right)$ or $O S p(8 \mid 4, \mathbf{R})$ respectively.

By locally asymptotic, we mean here that the boundary can consist of several components, each of which is asymptotic to $A d S_{5} \times S^{5}, A d S_{7} \times S^{4}$ or $A d S_{4} \times S^{7}$.

The compact factors of the above space-times cause no difficulty in the definition of this asymptotic behavior. The precise definitions of asymptotically $A d S_{3}$ and $A d S_{4}$ were given respectively in [76] and [77], and of asymptotically $A d S_{d+1}$, for general $d$, may be found, for example in $[78,79]$. We shall reproduce it here for the sake of completeness and clarity. In local adapted coordinates $x^{\mu}=\left(t, r, \theta^{i}\right)$, for $\mu=0, r, i$ and $i=1, \cdots, d-1$, a locally asymptotically $A d S_{d+1}$ metric $d s^{2}$ on a manifold $M$ takes the form,

$$
\begin{aligned}
& d s^{2}=d \hat{s}_{R}^{2}+h_{\mu \nu} d x^{\mu} d x^{\nu} \\
& d \hat{s}_{R}^{2}=-\left(1+\frac{r^{2}}{R^{2}}\right) d t^{2}+\left(1+\frac{r^{2}}{R^{2}}\right)^{-1} d r^{2}+r^{2} d \hat{s}_{S^{d-1}}^{2}
\end{aligned}
$$

Here, $d \hat{s}_{R}^{2}$ is the $S O(2, d)$-invariant metric on $A d S_{d+1}$ with radius $R$, expressed in terms of the $S O(d)$-invariant metric $d \hat{s}_{S^{d-1}}^{2}$ on $S^{d-1}$ with radius 1 . The boundary of $A d S_{d+1}$, and thus of $M$, is located at $r=\infty$. 
The metric $d s^{2}$ is locally asymptotic to the metric $d \hat{s}_{R}^{2}$ if the deformations away from $d \hat{s}_{R}^{2}$ (which are parametrized by $h_{\mu \nu}$ ) obey the following asymptotics as $r \rightarrow \infty$,

$$
\begin{aligned}
h_{t t} & =r^{2-d} \gamma_{t t}\left(t, \theta^{i}\right)+\mathcal{O}\left(r^{1-d}\right) \\
h_{t r} & =r^{-1-d} \gamma_{t r}\left(t, \theta^{i}\right)+\mathcal{O}\left(r^{-2-d}\right) \\
h_{t i} & =r^{2-d} \gamma_{t i}\left(t, \theta^{i}\right)+\mathcal{O}\left(r^{1-d}\right) \\
h_{r r} & =r^{-2-d} \gamma_{r r}\left(t, \theta^{i}\right)+\mathcal{O}\left(r^{-3-d}\right) \\
h_{r i} & =r^{-1-d} \gamma_{r i}\left(t, \theta^{i}\right)+\mathcal{O}\left(r^{-2-d}\right) \\
h_{i j} & =r^{2-d} \gamma_{i j}\left(t, \theta^{i}\right)+\mathcal{O}\left(r^{1-d}\right)
\end{aligned}
$$

The reduced metric functions $\gamma_{\mu \nu}$ are allowed to be arbitrary functions of $t$ and $\theta^{i}$.

The notion we need next is that of asymptotic symmetry of $M$, a notion that found its origins in gauge theory, and was extended long ago to general relativity $[80,76,77]$. Simply put for the case at hand, the asymptotic symmetry of the asymptotically $A d S_{d+1}$ manifold $M$ is $S O(2, d)$. This may be understood as follows. Under the isometry $S O(2, d)$ of $A d S_{d+1}$, interior points of $A d S_{d+1}$ are mapped to interior points, while points on the boundary of $A d S_{d+1}$ are mapped to boundary points. The action of $S O(2, d)$ induces conformal transformations on the boundary of $A d S_{d+1}$. It therefore makes sense to associate an asymptotic symmetry with any asymptotically $A d S_{d+1}$ space-time, and this symmetry is precisely $S O(2, d)$. The group $S O(2, d)$ then transforms the form of the boundary conditions (5.2) into itself.

More mathematically, the asymptotic symmetry may be viewed as the quotient of the group of all diffeomorphisms $\operatorname{Diff}(M)$ of $M$ by the group $\operatorname{Diff}_{\infty}(M)$ of all diffeomorphisms that leave the asymptotic conditions (5.2) invariant. Thus one has $\operatorname{Diff}(M) / \operatorname{Diff}(M)=S O(2, d)$.

When the boundary of a connected locally asymptotically $A d S_{d+1}$ manifold counts several disconnected components, the asymptotic symmetry algebra remains a single copy of $S O(2, d)$. This follows from the fact that the imposition of asymptotic boundary conditions on the Killing vectors on one boundary component fixes the Killing vectors completely on all other boundary components.

Extending the concept of asymptotic symmetry to include supersymmetry [76] is achieved by collecting Killing vectors and Killing spinors, and considering the superalgebra of their asymptotic actions on the various fields on the manifold, such as the metric. In this way, the asymptotic symmetry of an asymptotically $A d S_{5} \times S^{5}$ manifold in Type IIB theory, and of asymptotically $A d S_{7} \times S^{4}$ and $A d S_{4} \times S^{7}$ manifolds in M-theory are $P S U(2,2 \mid 4), O S p\left(8^{*} \mid 4\right)$, and $O S p(8 \mid 4, \mathbf{R})$ respectively. 
Global charges, which give a representation of the algebra of global symmetries on the fields, are defined in terms of the asymptotic behavior of the fields and are insensitive to the sub-leading behavior in (5.2). As a result, the global charges must span a subalgebra of the asymptotic symmetry algebra. Thus, any symmetry of an asymptotically $A d S_{5} \times S^{5}$ solution in Type IIB theory, and of asymptotically $A d S_{7} \times S^{4}$ and $A d S_{4} \times S^{7}$ solutions in M-theory must be a subalgebra of $P S U(2,2 \mid 4), O S p\left(8^{*} \mid 4\right)$, or $O S p(8 \mid 4, \mathbf{R})$ respectively.

Note that the converse of the correspondence does not hold. Namely, it does not follow from the above arguments that every solution whose symmetry superalgebra $\mathcal{H}$ is a subalgebra of $P S U(2,2 \mid 4), O S p\left(8^{*} \mid 4\right)$, or $O S p(8 \mid 4, \mathbf{R})$, is necessarily respectively asymptotically $A d S_{5} \times S^{5}$, asymptotically $A d S_{7} \times S^{4}$ or asymptotically $A d S_{4} \times S^{7}$, and indeed, this converse property does not appear to hold.

\subsection{Construction of the space-time Ansatz}

Given the maximal bosonic subalgebra $\mathcal{H}_{\text {bos }}$ of one of the subalgebras $\mathcal{H}$, the corresponding space-time Ansatz is generally not unique. It is always possible to restrict, without loss of generality, the space-time Ansatz so that each component space of the Ansatz consists of a single orbit of each simple or $U(1)$-component of $\mathcal{H}_{\text {bos }}$. Families of different orbits may then be assembled using the remaining free parameters of the Ansatz. For example, the algebra $S O(3)$ may be realized on either $S^{2}$, or on $S^{3}$, since the latter is itself a group manifold, namely the double cover of $S O(3)$. The realization on $S^{3}$, however, is a special case of the realization on $S^{2}$, as $S^{3}$ may be viewed as the Hopf bundle over $S^{2}$. As a result, $S O(3)$ needs only be realized on $S^{2}$. Even after this simplification, however, the space-time component in the Ansatz may not be unique. Below we shall discuss precisely how this happens.

The simple and $U(1)$ components of $\mathcal{H}_{\text {bos }}$ that will be needed in our analysis may be read off from the classification of subalgebras $\mathcal{H}$ in (4.18) and (4.23), and their maximal bosonic subalgebras $\mathcal{H}_{\text {bos }}$ in Table 9 . They are $S O(n+1)$ for $n=0, \cdots, 7, S O(2, n)$ for $n=1,2,3,4,6, S U(n+1)$ for $n=1,2,3$ and $S U(1, n)$ for $n=1,2,3$. (Here, we have used the coincidences at low rank, listed in Appendix A, to replace the required $S p(2 n), S p(2 n, \mathbf{R})$ and $S O\left(2 n^{*}\right)$ by equivalent algebras in the $S O$ and $S U$ series.) The standard realizations of these symmetries on corresponding homogeneous spaces are as follows,

$$
\begin{array}{rlrl}
S O(n+1) & S^{n} & S O(n+1) / S O(n) \\
S O(2, n) & A d S_{n+1} & =S O(2, n) / S O(1, n) \\
S U(n+1) & C P_{n} & =S U(n+1) / S[U(1) \times U(n)] \\
S U(1, n) & C H_{n} & =S U(1, n) / S[U(1) \times U(n)]
\end{array}
$$


Here, $C P_{n}$ is (compact) complex projective space, while $C H_{n}$ is (non-compact) complex hyperbolic space-time (see for example [81], [82], and especially [83], page 282). Recall that $C P_{n}$ and $C H_{n}$ for $n \geq 2$ are symmetric spaces but, unlike the manifolds $S^{n}$ and $A d S_{n+1}$, they are not maximally symmetric. As a result, $C P_{n}$ and $C H_{n}$ will support only half of the maximal number of Killing spinors. This forces the dilaton to be constant, and the axion and 3-form field strengths to vanish for the corresponding supergravity solutions.

Several of these low-rank algebras admit, however, several equivalent and inequivalent realizations, which we shall now discuss. The algebra $S O(4)=S O(3) \oplus S O(3)$ may be realized on either $S^{3}$, or on $S^{2} \times S^{2}$, and these cases need to be considered separately, as they are inequivalent. Analogously, $S O(2,2)=S O(1,2) \oplus S O(1,2)$ could in principle be realized in two inequivalent ways as well, namely on $A d S_{3}$ or on $A d S_{2} \times A d S_{2}$. Unlike its compact counterpart, $A d S_{2} \times A d S_{2}$ cannot correspond to a physically acceptable space-time manifold, and will therefore be excluded from consideration.

The realization of $S O(5)=S p(4)$ on $S^{4}$ is unique, since we have $S^{4}=S p(4) /[S p(2) \times$ $S p(2)]$. The realization of $S O(6)=S U(4)$ may be either on $S^{5}$ or on $C P_{3}$. However, in the case of Type IIB supergravity, $C P_{3}$ produces a space that is not asymptotically $A d S_{5} \times S^{5}$, and is therefore to be excluded. In the case of M-theory, $C P_{3}$ is allowed for solutions asymptotic to $A d S_{4} \times S^{7}$, but not to $A d S_{7} \times S^{4}$. Similarly, its hyperbolic counterpart may be used for solutions asymptotic to $A d S_{7} \times S^{4}$, but not to $A d S_{4} \times S^{7}$.

Similarly, $S U(3)$ can in principle be realized on the space $S U(3) / S O(3)$ instead of $C P_{2}$. The space $S U(3) / S O(3)$ is a 5 -dimensional compact manifold which is topologically and metrically different from $S^{5}$, and can not lead to a solution which is asymptotic to $A d S_{5} \times S^{5}$. The manifold $S U(1,2) / S O(1,2)$ is 5 -dimensional with signature $(++---)$ and is thus not a viable candidate either. The space $S U(4) / S O(4)$, having dimension 9 , is excluded on dimensional grounds. Finally, space-times which contain the products $C P_{2} \times S^{1}$ and $\mathrm{CH}_{2} \times S^{1}$ may be warped into $S^{5}$ and $A d S_{5}$ respectively, so that the corresponding full space-time can be asymptotic to $A d S_{5} \times S^{5}$.

\subsection{Half-BPS solutions in Type IIB supergravity}

For half-BPS solutions asymptotic to $A d S_{5} \times S^{5}$ in Type IIB, the relevant superalgebra is $P S U(2,2 \mid 4)$. In Table 10 we list all simple and direct sum subalgebras $\mathcal{H}$ with 16 fermionic generators of $P S U(2,2 \mid 4)$, their maximal bosonic subalgebra $\mathcal{H}_{\text {bos }}$, the corresponding Ansatz for the space-time manifold of the solution, as derived in subsection 5.2, and in the last column entitled "brane" we list the corresponding intersecting and probe brane cases of

Tables 1 and 2. In each case, there is a base space of parameters which has either Euclidean 


\begin{tabular}{|c|c|c|c|c|}
\hline case & $\mathcal{H}$ & $\mathcal{H}_{\text {bos }}$ & space-time Ansatz & brane \\
\hline \hline I & $S U(2 \mid 4)$ & $S O(3) \oplus S O(6) \oplus S O(2)$ & $M_{2} \times S^{2} \times S^{5} \times S^{1}$ & \\
\hline I $^{*}$ & $S U(2 \mid 4) \oplus S U(2)$ & $S O(4) \oplus S O(6) \oplus S O(2)$ & $M_{1} \times S^{3} \times S^{5} \times S^{1}$ & \\
\hline II & $S U(1,1 \mid 4)$ & $S O(2,1) \oplus S O(6) \oplus S O(2)$ & $A d S_{2} \times S^{5} \times S^{1} \times \Sigma_{2}$ & \\
\hline II $^{*}$ & $S U(1,1 \mid 4) \oplus S U(1,1)$ & $S O(2,2) \oplus S O(6) \oplus S O(2)$ & $A d S_{3} \times S^{5} \times S^{1} \times \Sigma_{1}$ & 4 \\
\hline III & $S U(2,2 \mid 2)$ & $S O(2,4) \oplus S O(3) \oplus S O(2)$ & $A d S_{5} \times S^{2} \times S^{1} \times \Sigma_{2}$ & \\
\hline III $^{*}$ & $S U(2,2 \mid 2) \oplus S U(2)$ & $S O(2,4) \oplus S O(4) \oplus S O(2)$ & $A d S_{5} \times S^{3} \times S^{1} \times \Sigma_{1}$ & 6 \\
\hline IV & $O S p\left(4^{*} \mid 4\right)$ & $S O(2,1) \oplus S O(3) \oplus S O(5)$ & $A d S_{2} \times S^{2} \times S^{4} \times \Sigma_{2}$ & 1,2 \\
\hline Va & $O S p(4 \mid 4, \mathbf{R})$ & $S O(2,3) \oplus S O(3)^{2}$ & $A d S_{4} \times S^{2} \times S^{2} \times \Sigma_{2}$ & 5 \\
\hline Vb & $O S p(4 \mid 4, \mathbf{R})$ & $S O(2,3) \oplus S O(4)$ & $A d S_{4} \times S^{3} \times \Sigma_{3}$ & \\
\hline VIa & $P S U(2 \mid 2)^{2} \oplus \mathbf{R}$ & $S O(4) \oplus S O(4) \oplus \mathbf{R}$ & $M_{3} \times \mathbf{R} \times S^{3} \times S^{3}$ & \\
\hline VIb & $P S U(2 \mid 2)^{2} \oplus \mathbf{R}$ & $S O(4) \oplus S O(3)^{2} \oplus \mathbf{R}$ & $M_{2} \times \mathbf{R} \times S^{3} \times S^{2} \times S^{2}$ & \\
\hline VIIa & $P S U(1,1 \mid 2)^{2} \oplus U(1)$ & $S O(2,2) \oplus S O(4) \oplus S O(2)$ & $A d S_{3} \times S^{3} \times S^{1} \times \Sigma_{3}$ & 3 \\
\hline VIIb & $P S U(1,1 \mid 2)^{2} \oplus U(1)$ & $S O(2,2) \oplus S O(3)^{2} \oplus S O(2)$ & $A d S_{3} \times S^{2} \times S^{2} \times S^{1} \times \Sigma_{2}$ & \\
\hline VIII & $S U(2 \mid 3) \oplus S U(2 \mid 1)$ & $S U(3) \oplus S O(3)^{2} \oplus S O(2)^{2}$ & $M_{1} \times C P_{2} \times S^{3} \times S^{1} \times S^{1}$ & \\
\hline IX & $S U(1,1 \mid 3) \oplus S U(1,1 \mid 1)$ & $S O(2,2) \oplus S U(3) \oplus S O(2)^{2}$ & $A d S_{3} \times C P_{2} \times S^{1} \times S^{1} \times \Sigma_{1}$ & \\
\hline X & $S U(1,2 \mid 2) \oplus S U(1 \mid 2)$ & $S U(1,2) \oplus S U(2)^{2} \oplus S O(2)^{2}$ & $M_{1} \times C H_{2} \times S^{3} \times S^{1} \times S^{1}$ & \\
\hline \hline
\end{tabular}

Table 10: Subalgebras of $P S U(2,2 \mid 4)$ with 16 fermionic generators

or Minkowski signature and over which the products of the symmetric spaces are fibered. The Euclidean base spaces of dimension $d$ are denoted by $\Sigma_{d}$, while the Minkowski ones are denoted by $M_{d}$.

The superalgebra $\mathcal{H}$ is a maximal subalgebra of $P S U(2,2 \mid 4)$ for cases $I^{*}$, II*, III*, IV, Va, Vb, VIII, IX, and X. Cases I, II, and III may be obtained from cases I*, II*, III* by removing their respective purely bosonic invariant subalgebras $S U(2), S U(1,1)$, and $S U(2)$. The space of solutions for cases $\mathrm{I}^{*}$, II*, III* forms a sub-family (with enhanced purely bosonic symmetries) of the space of solutions for cases I, II, and III, respectively. Note that it is possible as well to have solutions with partially enhanced bosonic symmetry, associated with a non-trivial subalgebra of the corresponding purely bosonic algebra. For $S U(2)$, we can have a $U(1)$ subalgebra, while for $S U(1,1)$, the subalgebra can be $U(1), S O(1,1)$ or even the 2-dimensional algebra consisting of a boost and a translation.

Cases VIa, VIb, and cases VIIa, VIIb have Abelian purely bosonic invariant subalgebras, and are listed in Table 10 with one factor of $\mathbf{R}$ or $U(1)$ respectively. This factor is not required by the mandate of 16 supersymmetries, and could be dropped, resulting in less bosonic symmetry for the space-time Ansatz and corresponding solutions. On the other 
hand, solutions could exist with an extra factor of $\mathbf{R}$ or $U(1)$, since the maximal subalgebras of $P S U(2,2 \mid 4)$ contain two factors of $\mathbf{R}$ or $U(1)$ respectively.

Several of the cases in Table 10 correspond to existing families of known fully backreacted half-BPS solutions in Type IIB supergravity. For other cases, where no non-trivial solutions are known yet, the knowledge of a suitable superalgebra $\mathcal{H}$ will lead us to suggest the existence of new families of half-BPS solutions. We shall discuss each case in turn below.

- Cases I and I*: The exact supergravity solutions for case I are discussed in $[5,84,85,86]$. They are dual to local gauge invariant half-BPS operators in $\mathcal{N}=4$ super-Yang-Mills. Case I* would correspond to a sub-family of solutions with enhanced bosonic symmetry; it is not known whether any such non-trivial solutions exist.

- Cases II and II*: No non-trivial solutions are known to exist for either case. The bosonic symmetries and space-time Ansatz of case $\mathrm{II}^{*}$ precisely match those of the D7/D3 intersection case 4 in Table 1, and corresponding D7 probe case 4 of Table 2. A more detailed discussion is postponed until subsection 5.4.

- Cases III and III*: No non-trivial solutions are known to exist for either case, but these cases are presently under investigation in [87]. The bosonic symmetries and space-time Ansatz of case III* precisely match those of the D7/D3 intersection case 6 in Table 1, and corresponding D7 probe case 6 of Table 2. A more detailed discussion is postponed until subsection 5.4 .

- Case IV: This case corresponds to holographic duals of supersymmetric Wilson loops $[88,47]$, and is associated with the intersecting brane and probe brane cases 1 , and 2 in Tables 1 and 2. The fully back-reacted supergravity solutions were obtained exactly in [9], following earlier work in $[52,53]$. Applications to Wilson loops at strong coupling in the CFT is discussed in [54].

- Case V: Case Va corresponds to the holographic duals of 2+1 dimensional defects, and is associated with the intersecting brane and probe brane case 5 of Tables 1 and 2 . The fully back-reacted supergravity solutions were obtained exactly in $[7,8]$, following earlier work in [56]. No non-trivial solutions are known for Case Vb; since there is an $A d S_{4}$ factor, such solutions should be dual to an interface or defect.

- Case VI: Case VIa is the "bubbling AdS" solution of [5], which is dual to local halfBPS operators. No non-trivial half-BPS supergravity solutions are known to exist to Case VIb. (Note that the supergroup can in principle be realized on the space $M_{2} \times S^{2} \times S^{2} \times S^{2} \times S^{2}$, but this space cannot be asymptotic to $A d S_{5} \times S^{5}$.) 
- Case VII: Case VIIa corresponds to the holographic dual of half-BPS surface operators, and is associated with the intersecting brane and probe brane case 3 of Tables 1 and 2. The solutions have been discussed recently in [33, 34]. No non-trivial half-BPS supergravity solutions are known to exist to Case VIIb. (Type IIB solutions with an $A d S_{3}$, but fewer than 16 supersymmetries, have been studied in [89] and [90].)

- Cases VIII, IX, and X: No supergravity solutions for these cases are presently known. If solutions exist, they will be qualitatively different from those of the other cases of Table 10. Since the spaces $\mathrm{CP}_{2}$ or $\mathrm{CH}_{2}$, which appear in their space-time Ansätze, support only half the maximal number of Killing spinors, the solutions are restricted to have constant dilaton and axion, and vanishing 3-form field strength. Since the parameter spaces $\Sigma_{1}$ of $M_{1}$ are only 1-dimensional, the reduced BPS equations may be too restrictive for solutions to exist. We leave this problem for future work.

A general argument [91], using the AdS/CFT correspondence with local operators, shows ${ }^{7}$ that the invariance superalgebra $\mathcal{H}$ for half-BPS solutions which are genuinely asymptotic to $A d S_{5} \times S^{5}$ (and not just locally asymptotic to $A d S_{5} \times S^{5}$ ) must have rank strictly less than the rank 6 of $P S U(2,2 \mid 4)$. The use of this argument leads us to conclude that cases I*, II*, III*, VIII, IX, and X cannot have solutions that are genuinely asymptotic to $A d S_{5} \times S^{5}$, though there appears to be no obstruction to the existence of locally asymptotic solutions. The argument also clarifies the natural occurrence in cases VIa, VIb of one factor of $\mathbf{R}$ (which gives $\operatorname{rank}(\mathcal{H})=5$ ), but not of two factors of $\mathbf{R}$, since such solutions would correspond to rank 6. A similar clarification emerges for the $U(1)$ factors of cases VIIa, and VIIb.

\subsection{On the existence of fully back-reacted D7 brane solutions}

Cases related to D7 branes in Type IIB appear to be especially intricate, and we shall devote the present subsection to an analysis of their existence. This concerns the intersections of D3 and D7 branes denoted as cases 4 and 6 in Table 1, and the related probe D7 brane cases 4 and 6 in Table 2. Case 6 is especially interesting since probe D7 branes may be used to introduce flavor into the AdS/CFT correspondence [50].

On the one hand, the nature of near-horizon limits of $1+1$ - or $3+1$-dimensional intersections of D7 branes with D3 branes, or of D7 branes viewed as probes in the $A d S_{5} \times S^{5}$ background provides evidence for the existence of corresponding half-BPS solutions. Case 4 in Table 1 corresponds to a 1+1-dimensional intersection of D7 branes and D3 branes.

\footnotetext{
${ }^{7}$ We are grateful to Shahin Sheikh-Jabbari for sharing this argument [91], and agreeing to its use here.
} 
The enhancement of the symmetries in the near-horizon limit, as well as the symmetry of the probe D7 brane of case 4 in Table 2, suggest a solution with bosonic symmetry $S O(2,2) \oplus S O(6) \oplus S O(2)$. Case 6 in Table 2 corresponds to a $3+1$-dimensional intersection of D7 branes and D3 branes, and similarly suggests the existence of a solution with bosonic symmetry $S O(2,4) \oplus S O(4) \oplus S O(2)$. On the other hand, D7 branes produce flavor multiplets in the fundamental representation of the gauge group in the dual CFT, which produce a non-vanishing renormalization group $\beta$-function, break scaling and conformal invariance, and thus vitiate solutions with $A d S_{3}$ or $A d S_{5}$ factors on the gravity side. Arguments have indeed been presented in $[15,16,17]$ (following earlier work on D7 branes in $[18,19]$ ) that no such fully back-reacted solutions corresponding to D7 branes should exist.

Our superalgebra analysis has revealed the existence of case II* and case III* in Table 10, whose global bosonic symmetries, supersymmetries, and space-time structure all exactly match those suggested by D7 probe or D7/D3 intersecting brane analysis. The superalgebra, bosonic subalgebra, and space-time Ansatz for these cases are given as follows,

$$
\begin{array}{cccc}
\text { II }^{*} & S U(1,1 \mid 4) \oplus S U(1,1) & S O(2,2) \oplus S O(6) \oplus S O(2) & A d S_{3} \times S^{5} \times S^{1} \times \Sigma_{1} \\
\text { III }^{*} & S U(2,2 \mid 2) \oplus S U(2) & S O(2,4) \oplus S O(4) \oplus S O(2) & A d S_{5} \times S^{3} \times S^{1} \times \Sigma_{1}
\end{array}
$$

Both superalgebras above are subalgebras of $P S U(2,2 \mid 4)$, suggesting that fully back-reacted half-BPS solutions for the near-horizon limit of D7/D3 branes can exist.

Closer analysis of the superalgebra story reveals a further layer of subtlety. Both subalgebras above are not "minimal", but possess purely bosonic invariant subalgebras (respectively $S U(1,1)$ and $S O(3))$, which are not required by the mandate of 16 supersymmetries. Their removal leads to corresponding "general cases", namely II and III of Table 10,

$$
\begin{array}{clll}
\text { II } & S U(1,1 \mid 4) & S O(2,1) \oplus S O(6) \oplus S O(2) & A d S_{2} \times S^{5} \times S^{1} \times \Sigma_{2} \\
\text { III } & S U(2,2 \mid 2) & S O(2,4) \oplus S O(3) \oplus S O(2) & A d S_{5} \times S^{2} \times S^{1} \times \Sigma_{2}
\end{array}
$$

for which, based on superalgebra arguments alone, solutions should exist. Fully back-reacted half-BPS solutions for the near-horizon limit of D7/D3 branes would then have to emerge as a sub-family of solutions with enhanced bosonic symmetry. It is unclear whether such bosonic enhancement is fully consistent.

A further subtlety for case $\mathrm{II}^{*}$, is that the bosonic invariant Lie subalgebra $S U(1,1)$ is part of the isometry algebra of $A d S_{3}$, and thus part of the conformal algebra of the CFT dual. Removing this factor will destroy part of the conformal symmetry. Also, the superconformal algebra of case $\mathrm{II}^{*}$ is realized in a left-right asymmetric (or heterotic) way, with right-movers 
carrying all of the 16 supersymmetries. In the full string theory sigma model, this asymmetry will produce anomalies, and they in turn may destroy the solution at the string theory level.

Perhaps the most convincing evidence that cases $\mathrm{II}^{*}$ and III* cannot support half-BPS solutions with genuine asymptotic $A d S_{5} \times S^{5}$ behavior result from applying the arguments of [91] (see the last paragraph of section 5.3). The Lie superalgebras $\mathcal{H}$ corresponding to cases II* and III* both have rank 6 , which is not allowed for genuine $A d S_{5} \times S^{5}$ asymptotics by the arguments of [91]. The only way to lower the rank is to remove completely the corresponding purely bosonic invariant subalgebra, thus lowering the symmetries to those of cases II and III respectively. But in doing so, the removal of the bosonic invariant subalgebra vitiates the existence of the corresponding fully back-reacted near-horizon D7 brane solutions. For case III, the search for solutions is presently being carried out in [87].

\subsection{Half-BPS solutions in M-theory}

The space-time Ansätze in M-theory are constructed in a similar manner to those of Type IIB supergravity discussed in section 5.2. The symmetries of the $A d S_{7} \times S^{4}$ space-time are encoded in the superalgebra of $O S p\left(8^{*} \mid 4\right)$. The simple and direct sum subalgebras with 16 fermionic generators were derived in Section 4 and are listed in Table 11. The notation is the same as in the previous section dealing with the Type IIB case.

\begin{tabular}{|c|c|c|c|c|}
\hline case & $\mathcal{H}$ & $\mathcal{H}_{\text {bos }}$ & space-time Ansatz & brane \\
\hline \hline I & $O S p\left(8^{*} \mid 2\right)$ & $S O(2,6) \oplus S O(3)$ & $A d S_{7} \times S^{2} \times \Sigma_{2}$ & \\
\hline I $^{*}$ & $O S p\left(8^{*} \mid 2\right) \oplus S p(2)$ & $S O(2,6) \oplus S O(4)$ & $A d S_{7} \times S^{3} \times \Sigma_{1}$ & \\
\hline II & $O S p\left(4^{*} \mid 4\right)$ & $S O(2,1) \oplus S O(3) \oplus S O(5)$ & $A d S_{2} \times S^{2} \times S^{4} \times \Sigma_{3}$ & \\
\hline II $^{*}$ & $O S p\left(4^{*} \mid 4\right) \oplus S O\left(4^{*}\right)$ & $S O(2,2) \oplus S O(4) \oplus S O(5)$ & $A d S_{3} \times S^{3} \times S^{4} \times \Sigma_{1}$ & \\
\hline IIIa & $S U(4 \mid 2)$ & $S O(6) \oplus S O(3) \oplus S O(2)$ & $M_{3} \times S^{5} \times S^{2} \times S^{1}$ & \\
\hline IIIb & $S U(1,3 \mid 2)$ & $S U(1,3) \oplus S O(3) \oplus S O(2)$ & $M_{2} \times C H_{3} \times S^{2} \times S^{1}$ & \\
\hline IIIc & $S U(2,2 \mid 2)$ & $S O(2,4) \oplus S O(3) \oplus S O(2)$ & $A d S_{5} \times S^{2} \times S^{1} \times \Sigma_{3}$ & 3 \\
\hline IV & $O S p\left(4^{*} \mid 2\right) \oplus O S p\left(4^{*} \mid 2\right)$ & $S O(2,2) \oplus S O(4) \oplus S O(4)$ & $A d S_{3} \times S^{3} \times S^{3} \times \Sigma_{2}$ & $1,2,5$ \\
\hline V & $O S p\left(2^{*} \mid 2\right) \oplus O S p\left(6^{*} \mid 2\right)$ & $S U(1,3) \oplus S O(4) \oplus S O(2)$ & $M_{1} \times C H_{3} \times S^{3} \times S^{1}$ & \\
\hline \hline
\end{tabular}

Table 11: Subalgebras of $O S p\left(8^{*} \mid 4\right)$ with 16 fermionic generators.

The symmetries of $A d S_{4} \times S^{7}$ space-time are encoded in the superalgebra of $O S p(8 \mid 4, \mathbf{R})$. Its simple and direct sum subalgebras with 16 fermionic generators are listed in Table 12. As in the case of Type IIB, there are several cases where corresponding half-BPS solutions are known. There are also cases, however, where the existence of solutions suggested by 
probe-brane arguments is not backed up by the superalgebra structure. The remaining cases provide evidence for the existence of new half-BPS solutions in M-theory. We shall now discuss these cases in turn.

\begin{tabular}{|c|c|c|c|c|}
\hline case & $\mathcal{H}$ & $\mathcal{H}_{\text {bos }}$ & space-time Ansatz & brane \\
\hline \hline $\mathrm{VIa}$ & $S U(4 \mid 2)$ & $S O(6) \oplus S O(3) \oplus S O(2)$ & $M_{3} \times S^{5} \times S^{2} \times S^{1}$ & \\
\hline $\mathrm{VIb}$ & $S U(4 \mid 2)$ & $S O(6) \oplus S O(3) \oplus S O(2)$ & $M_{2} \times C P_{3} \times S^{2} \times S^{1}$ & \\
\hline $\mathrm{VIc}$ & $S U(4 \mid 1,1)$ & $S O(6) \oplus S O(1,2) \oplus S O(2)$ & $A d S_{2} \times S^{5} \times S^{1} \times \Sigma_{3}$ & 4 \\
\hline $\mathrm{VII}$ & $O S p(4 \mid 2, \mathbf{R}) \oplus O S p(4 \mid 2, \mathbf{R})$ & $S O(2,2) \oplus S O(4) \oplus S O(4)$ & $A d S_{3} \times S^{3} \times S^{3} \times \Sigma_{2}$ & $1,2,5$ \\
\hline $\mathrm{VIII}$ & $O S p(4 \mid 4, \mathbf{R})$ & $S O(4) \oplus S O(2,3)$ & $A d S_{4} \times S^{2} \times S^{2} \times \Sigma_{3}$ & \\
\hline VIII* & $O S p(4 \mid 4, \mathbf{R}) \oplus S O(4)$ & $S O(4)^{2} \oplus S O(2,3)$ & $A d S_{4} \times S^{3} \times S^{3} \times \Sigma_{1}$ & \\
\hline $\mathrm{IX}$ & $O S p(8 \mid 2, \mathbf{R})$ & $S O(8) \oplus S O(2,1)$ & $A d S_{2} \times S^{7} \times \Sigma_{2}$ & \\
\hline $\mathrm{IX}$ & $O S p(8 \mid 2, \mathbf{R}) \oplus S p(2, \mathbf{R})$ & $S O(8) \oplus S O(2,2)$ & $A d S_{3} \times S^{7} \times \Sigma_{1}$ & \\
\hline $\mathrm{X}$ & $O S p(5 \mid 2, \mathbf{R}) \oplus O S p(3 \mid 2, \mathbf{R})$ & $S O(2,2) \oplus S O(5) \oplus S O(3)$ & $A d S_{3} \times S^{4} \times S^{2} \times \Sigma_{2}$ & \\
\hline $\mathrm{XIa}$ & $O S p(6 \mid 2, \mathbf{R}) \oplus O S p(2 \mid 2, \mathbf{R})$ & $S O(2,2) \oplus S O(6) \oplus S O(2)$ & $A d S_{3} \times S^{5} \times S^{1} \times \Sigma_{2}$ & \\
\hline $\mathrm{XIb}$ & $O S p(6 \mid 2, \mathbf{R}) \oplus O S p(2 \mid 2, \mathbf{R})$ & $S O(2,2) \oplus S O(6) \oplus S O(2)$ & $A d S_{3} \times C P_{3} \times S^{1} \times \Sigma_{1}$ & \\
\hline $\mathrm{XII}$ & $O S p(7 \mid 2, \mathbf{R}) \oplus O S p(1 \mid 2, \mathbf{R})$ & $S O(2,2) \oplus S O(7)$ & $A d S_{3} \times S^{6} \times \Sigma_{2}$ & \\
\hline \hline
\end{tabular}

Table 12: Subalgebras of $O S p(8 \mid 4, \mathbf{R})$ with 16 fermionic generators

The superalgebra $\mathcal{H}$ is a maximal subalgebra of $O S p\left(8^{*} \mid 4\right)$ for cases $\mathrm{I}^{*}, \mathrm{II}^{*}, \mathrm{IV}$, and $\mathrm{V}$, and of $O S p(8 \mid 4, \mathbf{R})$ for cases VII, VIII* IX $^{*}, \mathrm{X}$, XIa, XIb, and XII. Cases I, II, VIII, and IX may be obtained from cases I*, II*, VIII*, and $\mathrm{IX}^{*}$ by removing their respective purely bosonic invariant subalgebras $S p(2), S O\left(4^{*}\right), S O(4)$, and $S p(2, \mathbf{R})$. The space of solutions for cases

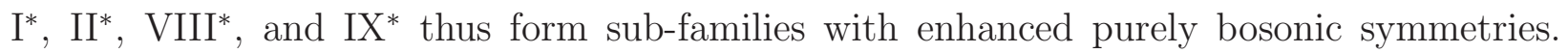
Note solutions may enjoy partial enhancement, associated with a non-trivial subalgebra, for example of $S O\left(4^{*}\right)$ or $S O(4)$. For cases IIIa, IIIb, IIIc, VIa, VIb, VIc, the subalgebra $\mathcal{H}$ is not maximal, and allows for enhancement by an Abelian $U(1)$ or $\mathbf{R}$ algebra.

- Cases I and I*: No non-trivial half-BPS supergravity solutions are known. These cases are analogous to Case III of Type IIB in Table 10.

- Cases II and II*: No non-trivial half-BPS supergravity solutions are known. Solutions in M-theory with an $A d S_{2}$-factor have been obtained in [90], but their number of supersymmetries is fewer than 16 .

- Case III: Case IIIa corresponds to the "bubbling AdS" solution of [5], which are dual to local half-BPS operators in the 6-dimensional CFT. Whether the supergravity solution 
corresponding to case IIIb exists is not known at this point. Case IIIc corresponds to a four dimensional BPS defect in the 6-dimensional CFT. These solutions should be related to the ones of case IIIa, but have not been explicitly constructed.

- Case IV corresponds to the half-BPS defect solutions found in [10] which are associated with two dimensional defects in the six dimensional CFT and from the perspective of the $(0,2)$ theory are the analog of the Wilson loop operators of Type IIB. This case is associated with the intersecting brane and probe branes cases 1, 2 of Tables 3 and 4 .

- Case V: No non-trivial half-BPS supergravity solutions are known to exist. This case is analogous to case $\mathrm{X}$ of Type IIB in Table 10, and both the fact that the $\mathrm{CH}_{3}$ restricts the supersymmetry and the base space is only one dimensional indicate that the solution if it exists, will be highly constrained.

- Case VI: Case VIa corresponds to the "bubbling AdS" solutions of [5], which are dual to local half-BPS operators in the three dimensional CFT associated with the decoupling limit of the M2 brane worldvolume theory. Whether the supergravity solution corresponding to case VIb exists is presently not known. The supergravity solution corresponding to case VIc was found in [63].

- Case VII: This case also corresponds to the half-BPS defect solutions found in [10] which are associated with two dimensional defects in the three dimensional CFT, they are the analog of the half-BPS interface solutions of Type IIB. This case is associated with the intersecting brane case 2 in Table 3 and the probe brane case 5 in Table 5 .

- Cases VIII and VIII*: No non-trivial half-BPS supergravity solutions are known to exist. This case is analogous to Case III of Type IIB in Table 10. Its dual interpretation is a deformation of the M2 brane CFT which preserves conformal symmetry but breaks half the supersymmetry.

- Cases IX and IX*: No non-trivial half-BPS supergravity solutions are known to exist. Progress in this direction has been made, however, in [92]. Solutions in M-theory with an $A d S_{2}$-factor have been obtained in [90, 93, 94], but with fewer than 16 supersymmetries.

- Case X-XII: No non-trivial half-BPS supergravity solutions are known to exist. (Solutions in M-theory with an $A d S_{3}$ factor, but with fewer than 16 supersymmetries, have been obtained in $[95,96]$.) Cases X-XII are similar to Case VII as they all have 
an $A d S_{3}$ factor and a two dimensional base base. They differ by the choice of sphere factors. If these solutions exist they would be dual to supersymmetric defects in the M2-brane CFT preserving different R-symmetries.

The general argument of [91], which we have already used for Type IIB solutions in the last paragraphs of both sections 5.3, and 5.4, applies to M-theory as well. The ranks of both superalgebras $O S p\left(8^{*} \mid 4\right)$ and $O S p(8 \mid 4, \mathbf{R})$ is 6 . The argument of [91] implies that solutions corresponding to superalgebras $\mathcal{H}$ whose rank is equal to 6 cannot have genuinely asymptotic $A d S_{7} \times S^{4}$ or $A d S_{4} \times S^{7}$ behavior, and rules out such solutions for the cases I $^{*}$, II* $, \mathrm{IV}, \mathrm{V}, \mathrm{VII}$, VIII*, IX*, XIa, and XIb. Of course, solutions which are locally asymptotic to $A d S_{7} \times S^{4}$ or $A d S_{4} \times S^{7}$ are allowed to exist. In fact, they are clearly known to exist, since they were derived, in explicit and exact local form in [10] for cases IV and VII, and in global form in [65] for case IV . 


\section{Conformal superalgebras with 16 supersymmetries}

The AdS/CFT correspondence maps the global symmetries of an AdS space-time solution onto the conformal supersymmetries of the dual CFT, and vice-versa. In particular, for the maximally supersymmetric case, the superalgebras $P S U(2,2 \mid 4), O S p\left(8^{*} \mid 4\right)$ and $O S p(8 \mid 4, \mathbf{R})$ are the symmetry algebras of respectively the $A d S_{5} \times S^{5}, A d S_{7} \times S^{4}$, and $A d S_{4} \times S^{7}$ solutions, but are also the conformal superalgebras of their respective dual CFTs, namely $\mathcal{N}=4 \mathrm{SYM}$, $\mathrm{CFT}_{6}$, and $\mathrm{CFT}_{3}$. The AdS/CFT map between global symmetries extends to any half-BPS supergravity solution (as classified in Sections 4 and 5), and any such solution should have a field theoretic dual with matching symmetry. The dual field theory can be a genuine CFT, or it can exhibit renormalization group flow behavior. In the first case, the symmetry of the half-BPS solution will translate to a superconformal symmetry on the CFT side. All possible superconformal symmetries in arbitrary space-time dimensions have been classified in [23] in terms of conformal superalgebras $\mathcal{C}$. A conformal superalgebra $\mathcal{C}$ in space-dimension $d$ is such that its maximal bosonic subalgebra has a factor of the conformal algebra $S O(2, d)$.

In this section, we shall review the classification of conformal superalgebras in [23], and derive its implications for the half-BPS case. In particular, we shall show that all of the solutions whose existence was advocated in Section 5 should have genuine CFT duals, although some solutions will be dual to the somewhat special case of "0-dimensional conformal field theory". It will be convenient to separate the cases with $d \geq 3, d=2$ and $d=1$.

\subsection{CFT space-time dimension $d \geq 3$}

For CFT space-time dimension $d$ larger than 2, Table 13 below lists the possible conformal superalgebras $\mathcal{C}$ with 16 fermionic generators of [23], together with their maximal bosonic subalgebra $\mathcal{C}_{\text {bos }}$, as a function of $d$.

\begin{tabular}{|c||c|c||c||c|}
\hline Dimension & $\mathcal{C}$ & $\mathcal{C}_{\text {bos }}$ & Type IIB & M-theory \\
\hline \hline$d=3$ & $O S p(4 \mid 4, \mathbf{R})$ & $S O(2,3) \oplus S O(4)$ & Va,Vb & VIII, VIII* \\
\hline$d=4$ & $S U(2,2 \mid 2)$ & $S O(2,4) \oplus S O(3) \oplus S O(2)$ & III, III* & IIIc \\
\hline$d=5$ & $F(4 ; 2)$ & $S O(2,5) \oplus S L(2, \mathbf{R})$ & none & none \\
\hline$d=6$ & $O S p\left(8^{*} \mid 2\right)$ & $S O(2,6) \oplus S O(3)$ & & I, I* $^{*}$ \\
\hline \hline
\end{tabular}

Table 13: Conformal superalgebras in $d \geq 3$ and corresponding cases of Section 5 .

In the rightmost two columns of Table 13 we list the cases of Type IIB supergravity and M-theory solutions of Section 5 on which each conformal superalgebra $\mathcal{C}$ may be realized as 
invariance algebra (namely the superalgebra $\mathcal{H}=\mathcal{C}$ of the classification in Section 5).

For $d=5$, the requirement that the superalgebra be conformal forces the unique real form $F(4 ; 2)$ of the exceptional superalgebra $F(4)$. The presence, for this real form, of the extra non-compact algebra $S L(2, \mathbf{R})$ implies that $F(4 ; 2)$ cannot be realized on $A d S_{6} \times M$ with $M$ compact. (One compact option is excluded as follows. The algebra $S L(2, \mathbf{R})$ is, of course, the isometry of the non-compact Poincaré upper half plane $H_{2}$. The quotient of $H_{2}$ by a hyperbolic Kleinian group produces a compact Riemann surface, but the quotienting destroys the supersymmetry.) Since no supergravity solution of the form $A d S_{6} \times M$ with $M$ compact exists, we write "none" in Table 13 for this case.

When no corresponding case of Section 5 is available, the relevant entry in either one of the last two columns in Table 13 is left blank. Table 13 reveals that in $d=6$ for the Type IIB, and in $d=4$ for M-theory, no cases of the classification of Section 5 are available, so that no corresponding half-BPS solutions exist which are asymptotic respectively to $A d S_{5} \times S^{5}$, $A d S_{4} \times S^{7}$, or $A d S_{7} \times S^{4}$, and any supergravity solutions corresponding to these cases must exhibit different asymptotics. On the CFT side, this implies result implies that the dual CFT cannot be obtained as a deformation of the vacuum via a "sufficiently local operator" (which is an operator defined to obey cluster decomposition with any set of local operators).

\subsection{CFT space-time dimension $d=2$}

For CFT space-time dimension $d=2$, the conformal algebra is not simple, but instead given by $S O(2,2)=S O(1,2) \oplus S O(1,2)$. As a result, the corresponding conformal superalgebras $\mathcal{C}$ with 16 fermionic generators are direct sums of a left superalgebra $\mathcal{C}^{L}$, and a right superalgebra $\mathcal{C}^{R}$. As is well-known, these finite-dimensional conformal and superconformal algebras for $d=2$ are enhanced to their full infinite-dimensional counterparts [23], with corresponding infinite-dimensional asymptotic symmetry of the associated supergravity solutions [76].

\begin{tabular}{|c||c|c|}
\hline $\mathcal{C}^{L, R}$ & $\mathcal{C}_{\text {bos }}^{L, R}$ & \# fermions \\
\hline \hline$O S p(m \mid 2, \mathbf{R})$ & $S O(2,1) \oplus S O(m)$ & $2 m$ \\
\hline$O S p\left(4^{*} \mid 2 m\right)$ & $S O(2,1) \oplus S O(3) \oplus S p(2 n)$ & $8 m$ \\
\hline$S U(1,1 \mid m)$ & $S O(2,1) \oplus S U(m) \oplus S O(2)$ & $4 m$ \\
\hline$G(3 ; 0)$ & $S O(2,1) \oplus G_{2}$ & 14 \\
\hline$F(4 ; 0)$ & $S O(2,1) \oplus S O(7)$ & 16 \\
\hline$D(2,1 ; c ; 0)$ & $S O(2,1) \oplus S O(4)$ & 8 \\
\hline \hline
\end{tabular}

Table 14: Conformal superalgebra factors for $d=2$ 
We begin by recalling, in Table 14, the classification of the conformal superalgebras of [23] for $d=2$, with arbitrary number of fermionic generators. The most general $d=2$ finite-dimensional conformal superalgebra $\mathcal{C}$ is given by a direct $\operatorname{sum} \mathcal{C}=\mathcal{C}^{L} \oplus \mathcal{C}^{R}$, with both $\mathcal{C}^{L}$ and $\mathcal{C}^{R}$ selected from Table 14 so that the total number of fermionic generators is 16.

Note that $\mathcal{C}^{L}$ and $\mathcal{C}^{R}$ may differ from one another in this construction, as they indeed do for the first seven cases in Table 15. One might refer to these cases as "heterotic", since $\mathcal{C}^{L}$ and $\mathcal{C}^{R}$ act on left-moving and right-moving degrees of freedom on the boundary CFT. Note that we find cases where both $\mathcal{C}^{L}$ and $\mathcal{C}^{R}$ are superalgebras, such as case II* $^{*}$ in M-theory, as well as other cases, like II* $^{*}$ in Type IIB, where $\mathcal{C}^{R}$ is the a purely bosonic algebra.

In Table 15, we lists conformal superalgebras $\mathcal{C}$, together with their maximal bosonic subalgebras $\mathcal{C}_{\text {bos }}$, for which there exists at least one corresponding case of half-BPS solutions in Section 5, either for Type IIB or for M-theory. The corresponding cases are indicated in the rightmost two columns of Table 15. Blank entries signify again that any supergravity half-BPS solutions must have asymptotics different from the respective maximally supersymmetric space-times, and that no such solutions are known at present.

\begin{tabular}{|c||c|c||c||}
\hline \multicolumn{1}{|c||}{$=\mathcal{C}_{L} \oplus \mathcal{C}_{R}$} & $\mathcal{C}_{\text {bos }}$ & Type IIB & M-theory \\
\hline \hline$S U(1,1 \mid 4) \oplus S U(1,1)$ & $S O(2,2) \oplus S O(6) \oplus S O(2)$ & II $^{*}$ & \\
\hline$S U(1,1 \mid 3) \oplus S U(1,1 \mid 1)$ & $S O(2,2) \oplus S U(3) \oplus S O(2)^{2}$ & IX & \\
\hline$O S p\left(4^{*} \mid 4\right) \oplus S O\left(4^{*}\right)$ & $S O(2,2) \oplus S O(4) \oplus S p(4)$ & & II $^{*}$ \\
\hline$O S p(5 \mid 2, \mathbf{R}) \oplus O S p(3 \mid 2, \mathbf{R})$ & $S O(2,2) \oplus S O(5) \oplus S O(3)$ & & X \\
\hline$O S p(6 \mid 2, \mathbf{R}) \oplus O S p(2 \mid 2, \mathbf{R})$ & $S O(2,2) \oplus S O(6) \oplus S O(2)$ & & XIa,XIb \\
\hline$O S p(7 \mid 2, \mathbf{R}) \oplus O S p(1 \mid 2, \mathbf{R})$ & $S O(2,2) \oplus S O(7)$ & & XII \\
\hline$O S p(8 \mid 2, \mathbf{R}) \oplus S p(2, \mathbf{R})$ & $S O(2,2) \oplus S O(8)$ & & IX $^{*}$ \\
\hline \hline$P S U(1,1 \mid 2) \oplus P S U(1,1 \mid 2)$ & $S O(2,2) \oplus S O(3) \oplus S O(3)$ & VIIa, VIIb & \\
\hline$O S p(4 \mid 2, \mathbf{R}) \oplus O S p(4 \mid 2, \mathbf{R})$ & $S O(2,2) \oplus S O(4) \oplus S O(4)$ & & VII $^{||||}$ \\
\hline$O S p\left(4^{*} \mid 2\right) \oplus O S p\left(4^{*} \mid 2\right)$ & $S O(2,2) \oplus S O(4) \oplus S O(4)$ & & IV \\
\hline$D(2,1 ; c) \oplus D(2,1 ; c)$ & $S O(2,2) \oplus S O(4) \oplus S O(4)$ & & Sect. 7.2 \\
\hline \hline
\end{tabular}

Table 15: Conformal superalgebras in $d=2$ with corresponding cases of Section 5 .

On the last line of Table 15, $c$ is an arbitrary real non-vanishing parameter. The case $c=-2,-1 / 2$ coincides with the first line of Table 15 , since $D(2,1 ; c ; 0)=O S p\left(4^{*} \mid 2\right)$ for these values of $c$, while the case $c=1$ coincides with the second line in Table 15 , since $D(2,1 ; 1 ; 0)=O S p(4 \mid 2, \mathbf{R})$. The cases $c \neq 1,-2,-1 / 2$ in M-theory correspond to known solutions $[20,10]$, which will be discussed in detail in Section 7.2. 
The remaining conformal superalgebras $\mathcal{C}=\mathcal{C}^{L} \oplus \mathcal{C}^{R}$ with 16 fermionic generators are,

$$
\begin{aligned}
& P S U(1,1 \mid 2) \oplus D(2,1 ; c ; 0) \quad O S p(4 \mid 2, \mathbf{R}) \oplus P S U(1,1 \mid 2) \quad O S p(4 \mid 2, \mathbf{R}) \oplus O S p\left(4^{*} \mid 2\right) \\
& O S p(4 \mid 2, \mathbf{R}) \oplus D(2,1 ; c ; 0) \quad O S p(2 \mid 2, \mathbf{R}) \oplus S U(1,1 \mid 3) \quad O S p(1 \mid 2, \mathbf{R}) \oplus G(3 ; 0) \\
& O S p\left(4^{*} \mid 2\right) \oplus D(2,1 ; c ; 0) \quad O S p\left(4^{*} \mid 2\right) \oplus P S U(1,1 \mid 2) \quad S O(2,1) \oplus F(4 ; 0)
\end{aligned}
$$

There are no known corresponding cases of Section 5, so that any CFTs with these symmetries cannot be obtained from the maximally supersymmetric ones by the insertion of a "sufficiently local operator".

\subsection{CFT space-time dimension $d=1$}

Table 16 lists the possible conformal superalgebras for $d=1$ of [23], together with their maximal bosonic subalgebras, and the AdS-dual cases, if any, they correspond to.

\begin{tabular}{|c||c|c||c||}
\hline $\mathcal{C}$ & $\mathcal{C}_{\text {bos }}$ & IIB case & M case \\
\hline \hline$O S p(8 \mid 2, \mathbf{R})$ & $S O(2,1) \oplus S O(8)$ & & IX \\
\hline$O S p\left(4^{*} \mid 4\right)$ & $S O(2,1) \oplus S O(3) \oplus S O(5)$ & IV & II \\
\hline$S U(1,1 \mid 4)$ & $S O(2,1) \oplus S O(6) \oplus S O(2)$ & II & VIc \\
\hline$F(4)$ & $S O(2,1) \oplus S O(7)$ & & \\
\hline \hline
\end{tabular}

Table 16: Conformal superalgebras in $d=1$ with corresponding cases of Section 5.

\subsection{CFT in dimension 0}

Cases I, I*, VIa, VIb, and VIII and X of Type IIB in Table 10, and cases IIIa, IIIb, V, VIa, and VIb of M-theory in Tables 11 and 12, may be interpreted as corresponding to 0-dimensional conformal field theory. The factor $M_{d}$ then contains the Minkowski signature time-direction. The solutions may be time-independent such as in [5], or become cosmological type solutions in view of time-dependent warping.

\subsection{Examples from $\mathcal{N}=4$ SYM/Type IIB duality}

As discussed in Section 2, the superconformal symmetry of a CFT can be reduced by the presence of operators of varying worldvolume dimension, including local operators, Wilson loops, and operators associated with defects, interfaces, and domain walls. An operator of worldvolume dimension $d$ is expected to preserve the conformal algebra $S O(2, d)$, associated 
with the conformal transformations which leave the operator invariant. For half-BPS operators, the symmetry algebra has to contain 16 fermionic generators and form a superalgebra. Perturbing the CFT by any such operator will cause a deformation of the dual supergravity solution, with an associated reduction of the invariance superalgebra to a subalgebra $\mathcal{H}$. A CFT with conformal superalgebra $\mathcal{H}$ which is a subalgebra of $P S U(2,2 \mid 4)$ should be a deformation of $\mathcal{N}=4 \mathrm{SYM}$ by the insertion of some "sufficiently local operator". (Similarly a CFT with conformal superalgebra $\mathcal{H}$ which is a subalgebra of $O S p\left(8^{*} \mid 4\right), O S p(8 \mid 4, R)$ should be a deformation of $\mathrm{CFT}_{6}$, and $\mathrm{CFT}_{3}$ respectively.)

For a conformal superalgebra $\mathcal{H}$ which is not a subalgebra of $P S U(2,2 \mid 4)$, the AdS/CFT correspondence predicts that no CFT deformation of $\mathcal{N}=4 \mathrm{SYM}$ exists which can break $\operatorname{PSU}(2,2 \mid 4)$ down to $\mathcal{H}$. This is because the dual AdS solution would then have to be locally asymptotic to $A d S_{5} \times S^{5}$, which in turn would contradict the results of Sections 4 and 5. While supergravity solutions with these symmetries may exist, they cannot be locally asymptotically $A d S_{5} \times S^{5}$. One immediate result from the below analysis is that there are no deformations of $\mathcal{N}=4 \mathrm{SYM}$ which yield a CFT of dimension greater than 4 .

The known half-BPS operators in $\mathcal{N}=4$ SYM provide the following examples,

- $P S U(2 \mid 2) \oplus P S U(2 \mid 2) \oplus \mathbf{R}$ corresponds to deformations of $\mathcal{N}=4$ SYM by local halfBPS operators. In [6] it was argued that the $P S U(2 \mid 2) \oplus P S U(2 \mid 2) \oplus \mathbf{R}$ half-BPS sector of $\mathcal{N}=4 \mathrm{SYM}$ can be described by the quantum mechanics of a system of fermions, which can be though of as a zero dimensional conformal field theory. In [5], it was shown that the dual geometries can be described in the same manner.

- $O S p\left(4^{*} \mid 4\right)$ corresponds to deformations of $\mathcal{N}=4$ SYM by half-BPS Wilson loop operators. In [47], it was argued that such operators are described by coupling a 1dimensional defect action to $\mathcal{N}=4 \mathrm{SYM}$. The authors essentially then reduced the problem to the 1-dimensional field theory of a fermion.

- $O S p(4 \mid 4, \mathbf{R})$ corresponds to the interface/defect CFTs discussed in [35, 48, 37, 38]. The 3-dimensional behavior is the most explicit in [37] where the CFT is constructed from three dimensional superfields and so the set of such field theories is governed by the space of three dimensional conformal SYM theories. The fourth direction is introduced as a gauge theory direction. The M-theory CFT has not been studied yet, although a three dimensional CFT is expected to exist.

- $S U(2,2 \mid 2)$ corresponds to coupling $\mathcal{N}=4 \mathrm{SYM}$ to additional hyper-multiplets [48, 50]. This corresponds to introducing flavor into the AdS/CFT correspondence. The resulting CFT is 4-dimensional. 


\section{Discussion}

In this section, we shall discuss two further issues which are directly related to the correspondence between Lie superalgebras and half-BPS supergravity solutions. The first is an analysis of when the 16 supersymmetries of a half-BPS solution may be enhanced. The second is an analysis of the family of M-theory solutions of [10] in the context of the Lie superalgebra - half-BPS supergravity solution correspondence.

\subsection{Enhanced supersymmetries}

On the one hand, all Type IIB and M-theory supergravity solutions with the maximal number of 32 supersymmetries are known to be given by the solutions $A d S_{5} \times S^{5}, A d S_{4} \times S^{7}$, and $A d S_{7} \times S^{4}$, with their associated invariance superalgebras respectively $P S U(2,2 \mid 4)$, $O S p(8 \mid 4, \mathbf{R})$ and $O S p\left(8^{*} \mid 4\right)$. On the other hand, we have classified in this paper all possible basic Lie superalgebras which are subalgebras of $\operatorname{PSU}(2,2 \mid 4), O S p(8 \mid 4, \mathbf{R})$ and $O S p\left(8^{*} \mid 4\right)$ with 16 fermionic generators, and argued that these provide a classification for half-BPS supergravity solutions asymptotic to $A d S_{5} \times S^{5}, A d S_{4} \times S^{7}$, and $A d S_{7} \times S^{4}$ respectively. Finally, we know that any solution which has 28 supersymmetries or more will automatically have the maximal number of 32 supersymmetries [4].

Taken together, the above results raise the question as to whether any of the half-BPS solutions advocated in this paper may actually exhibit enhanced supersymmetry, and possess a number of supersymmetry generators larger than 16, but smaller than 28.

The existence of such solutions within a family of half-BPS solutions with invariance superalgebra $\mathcal{H}$ will require the existence of a superalgebra $\mathcal{H}^{*}$ which is contained in the corresponding superalgebra with maximal symmetry, and obeys $\mathcal{H} \subset \mathcal{H}^{*}$. This condition is necessary for the existence of regular supergravity solutions, but we are not guaranteed that any non-trivial such solutions will exist.

It is straightforward to classify all such possible basic Lie superalgebras $\mathcal{H}^{*}$; remarkably, they are all simple superalgebras (i.e. they involve no non-trivial direct sums). The result is given in Table 17 .

Note that there may exist subalgebras of $P S U(2,2 \mid 4), O S p(8 \mid 4, \mathbf{R})$, or $O S p\left(8^{*} \mid 4\right)$, with more than 16 fermionic generators, but which do not contain a basic Lie superalgebra (or direct sum thereof) with 16 fermionic generators. For example, we have the embedding $S U(1,2 \mid 3) \subset P S U(2,2 \mid 4)$, but $S U(1,2 \mid 3)$ has no direct sum of basic Lie subalgebra with 16 fermionic generators. As a result, we do not expect $S U(1,2 \mid 3)$ to show up as an enhanced symmetry of a half-BPS solution, and the corresponding case is omitted from Table 17. 


\begin{tabular}{|c|c|c|c|c|}
\hline $\mathcal{H}$ & $\mathcal{H}^{*}$ & $\mathcal{G}$ & $\mathcal{H}_{\text {bos }}^{*}$ & space-time \\
\hline \hline$S U(2,2 \mid 2)$ & $S U(2,2 \mid 3)$ & $P S U(2,2 \mid 4)$ & $S O(2,4) \oplus U(3)$ & $A d S_{5} \times C P_{2} \times S^{1}$ \\
\hline$S U(2 \mid 4)$ & $S U(1,2 \mid 4)$ & $P S U(2,2 \mid 4)$ & $S U(1,2) \oplus U(4)$ & $C H_{2} \times S^{5} \times S^{1}$ \\
\hline$O S p\left(4^{*} \mid 4\right)$ & $O S p\left(6^{*} \mid 4\right)$ & $O S p\left(8^{*} \mid 4\right)$ & $S U(1,3) \oplus S O(5)$ & $M_{1} \times C H_{3} \times S^{4}$ \\
\hline$O S p(4 \mid 4, \mathbf{R})$ & $O S p(5 \mid 4, \mathbf{R})$ & $O S p(8 \mid 4, \mathbf{R})$ & $S O(2,3) \oplus S O(5)$ & $A d S_{4} \times S^{4} \times \Sigma_{3}$ \\
\hline$O S p(4 \mid 4, \mathbf{R})$ & $O S p(6 \mid 4, \mathbf{R})$ & $O S p(8 \mid 4, \mathbf{R})$ & $S O(2,3) \oplus S O(6)$ & $A d S_{4} \times S^{5} \times \Sigma_{2}$ \\
\hline$O S p(4 \mid 4, \mathbf{R})$ & $O S p(6 \mid 4, \mathbf{R})$ & $O S p(8 \mid 4, \mathbf{R})$ & $S O(2,3) \oplus S O(6)$ & $A d S_{4} \times C P_{3} \times \Sigma_{1}$ \\
\hline
\end{tabular}

Table 17: Lie superalgebras and associated space-times for enhanced half-BPS solutions

The case on the fourth line in Table 17 with $\mathcal{H}^{*}=O S p(5 \mid 4, \mathbf{R})$ has 20 fermionic generators, while all other cases have 24; they will correspond to supergravity solutions with respectively 20 and 24 supersymmetries (the latter being so-called three-quarter-BPS). Note that also $O S p(7 \mid 4, \mathbf{R})$ is a subalgebra of $O S p(8 \mid 4, \mathbf{R})$, but is has 28 supersymmetries, and will always lead to solutions invariant under the full $O S p(8 \mid 4, \mathbf{R})$ by [4].

The space-time forms on the first two lines involve genuine direct products of the components, as the total dimension 10 leaves no room for any extra free parameters. To recover the Minkowski metric, the $S^{1}$ component of the second line must be the time direction, and the solutions will have closed time-like curves. The space-times on the last four lines in Table 17 are generally warped over the parameter spaces $M_{1}, \Sigma_{1}, \Sigma_{2}$ and $\Sigma_{3}$. The case of the last line of Table 17 is related to the well-known $A d S_{4} \times C P_{3}$ solution to Type IIA supergravity [97], which has risen to prominence recently in view of its importance to the $A d S_{4} / C F T_{3}$ correspondence with maximal supersymmetry (see for example [60, 98]). In the $A d S_{4} \times C P_{3}$ solution to Type IIA supergravity, the product is direct, and no warping occurs. The relation with M-theory thus involves choosing $\Sigma_{1}$ to be simply $S^{1}$, and carrying out standard Kaluza-Klein reduction of M-theory on $S^{1}$ to Type IIA supergravity. It is an interesting question as to whether other such solutions exist as well, including those with actual warped products.

\subsection{Symmetries of the exact M-theory solutions}

The half BPS-solutions on $A d S_{3} \times S^{3} \times S^{3}$, either as a direct product with a flat Euclidean surface $E_{2}$ of [20] (see also [21, 22]), or warped over a Riemann surface $\Sigma$ of [10], actually consist of families of solutions indexed by a real parameter $c$. These families provide a beautiful illustration of the interplay between boundary asymptotic behavior of half-solutions and their superalgebra invariance, which we shall bring to the fore in this subsection. 
To show how the parameter $c$ emerges, we exhibit the metric of the solutions of [10],

$$
d s^{2}=\frac{\tilde{f}_{1}^{2}}{c_{1}^{2}} d s_{A d S_{3}}^{2}+\frac{\tilde{f}_{2}^{2}}{c_{2}^{2}} d s_{S^{3}}^{2}+\frac{\tilde{f}_{3}^{2}}{c_{3}^{2}} d s_{S^{3}}^{2}+d s_{\Sigma}^{2}
$$

Here, $d s_{A d S_{3}}^{2}$ and $d s_{S^{3}}^{2}$ stand for the invariant metrics on the corresponding spaces, normalized to unit radius. The metric factors $\tilde{f}_{i}$, as well as all the other supergravity fields of the solution, may be expressed in terms of the supersymmetry parameters $\varepsilon$ (or Killing spinors) of the solution (see Appendix D for the detailed relations).

The parameters $c_{i}$, for $i=1,2,3$, are real. As a result of the BPS equations they must be constant, and subject to the relation, $c_{1}+c_{2}+c_{3}=0$. Since a common multiplicative factor in the $c_{i}$ corresponds to an overall scale of the solution, there remains only a single intrinsic combination of the $c_{i}$, which we may take to be $c=c_{2} / c_{3}$. All the supergravity fields have a non-trivial dependence on $c$.

In Appendix D, it will be shown that the invariance superalgebra of any solution of [10] is $D(2,1 ; c ; 0) \oplus D(2,1 ; c ; 0)$ for any arbitrary value of $c \neq 0$. Here, $D(2 ; 1 ; c ; 0)$ is the real form of the exceptional superalgebra $D(2,1 ; c)$ whose maximal bosonic subalgebra is $S O(1,2) \oplus S O(4)$ (see Section 4.2). For general values of $c$, the supergravity solutions involve a factor of $A d S_{3}$, and are thus dual to a 1+1-dimensional CFT. For each value of $c$, the superalgebra $D(2,1 ; c ; 0) \oplus D(2,1 ; c ; 0)$ indeed corresponds to one of the superconformal algebras allowed in $1+1$ dimensions in the classification of [23].

The dual 1+1-dimensional CFT may be enlarged, however, to a defect/interface CFT in $2+1$ dimensions for $c=1$, and to a 2-dimensional surface operator CFT in $5+1$ dimensions for $c=-2,-1 / 2$. The corresponding supergravity solutions are precisely those discussed in the second bullet point of Section 3.6; they are summarized in Table 7.2, and associated parameter diagram in Figure 7.2. [Erratum: some of the superalgebras of the corresponding figure in the JHEP version of [10] were labeled incorrectly, and have been corrected here.]

\begin{tabular}{|c||c|c|c|c|}
\hline case & $c$ & includes & superalgebra of solution & maximal superalgebra \\
\hline \hline$c_{2}=c_{3}$ & 1 & $A d S_{4} \times S^{7}$ & $O S p(4 \mid 2, \mathbf{R}) \times O S p(4 \mid 2, \mathbf{R})$ & $O S p(8 \mid 4, \mathbf{R})$ \\
\hline$c_{3}=c_{1}$ & -2 & $A d S_{7} \times S^{4}$ & $O S p\left(4^{*} \mid 2\right) \times O S p\left(4^{*} \mid 2\right)$ & $O S p\left(8^{*} \mid 4\right)$ \\
\hline$c_{1}=c_{2}$ & $-1 / 2$ & $A d S_{7} \times S^{4}$ & $O S p\left(4^{*} \mid 2\right) \times O S p\left(4^{*} \mid 2\right)$ & $O S p\left(8^{*} \mid 4\right)$ \\
\hline
\end{tabular}

Table 18: M-theory solutions asymptotic to $A d S_{4} \times S^{7}$ or $A d S_{7} \times S^{4}$.

This may be seen as follows. Since the $c_{i}$ are constants, they are determined by the asymptotic behavior of the solution. We have the following special cases. 


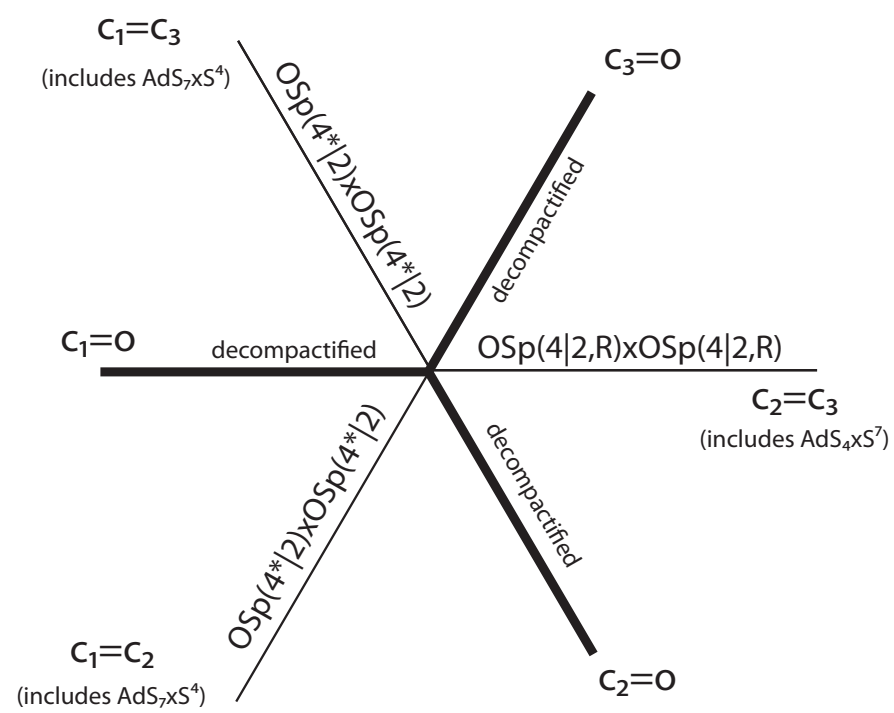

Figure 1: The space of parameters $c_{1}, c_{2}, c_{3}$ and corresponding limiting superalgebras.

- For $c=1$, the solutions are asymptotic to $A d S_{4} \times S^{7}$, and their invariance superalgebra is $O S p(4 \mid 2, \mathbf{R}) \oplus O S p(4 \mid 2, \mathbf{R})$, which coincides with $D(2,1 ; c ; 0) \oplus D(2,1 ; c ; 0)$ for $c=1$ (see Section 4.2), and is indeed contained in $\operatorname{OSp}(8 \mid 4, \mathbf{R})$.

- For $c=-2,-1 / 2$, the solutions are asymptotic to $A d S_{7} \times S^{4}$, and their invariance superalgebra is $O S p\left(4^{*} \mid 2\right) \oplus O S p\left(4^{*} \mid 2\right)$, which coincides with $D(2,1 ; c ; 0) \oplus D(2,1 ; c ; 0)$ for $c=-2,-1 / 2$ (see Section 4.2), and is indeed contained in $O S p\left(8^{*} \mid 4\right)$.

- For $c \neq 1,-2,-1 / 2$, the solutions are asymptotic to neither $A d S_{4} \times S^{7}$ nor $A d S_{7} \times S^{4}$. It will be shown in Appendix $\mathrm{C}$ that, correspondingly, the superalgebra of the solutions $D(2,1 ; c ; 0) \oplus D(2,1 ; c ; 0)$ is then a subalgebra of neither $O S p(8 \mid 4, \mathbf{R})$ nor $O S p\left(8^{*} \mid 4\right)$.

For each value of $c$, there exists a special solution for which $\tilde{f}_{1}=\tilde{f}_{2}=\tilde{f}_{3}=1$, and the metric $d s_{\Sigma}^{2}$ is flat Euclidean [20]. For these solutions, the product $A d S_{3} \times S^{3} \times S^{3} \times \Sigma$ is not warped over $\Sigma$, so that the space-time geometry is genuinely a direct product of these factors. The asymptotic behavior of the solution is that of $A d S_{3}$, and is dual to a 1+1-dimensional CFT. It would appear that this $A d S_{3}$ solution cannot be extended to be dual to an interface or surface operator, unlike for the case of $c=1,-2,-1 / 2$. It was shown in $[21,22]$ that these special solutions are invariant under $D(2,1 ; c ; 0) \oplus D(2,1 ; c ; 0)$ for all values of $c$. 


\section{Acknowledgments}

We gratefully acknowledge helpful conversations and correspondence with Murat Günaydin, Per Kraus, David Mateos, Kostas Skenderis, and Joris Van der Jeugt. We have benefited from various important questions and comments on the initial version of this paper by Jose Figueroa-O'Farrill, Jerome Gauntlett, Andy Royston, and especially Jaume Gomis and Shahin Sheikh Jabbari, and we wish to extend our warm thanks them.

E.D. and P.S. would like to thank the Laboratoire de Physique Théorique et Hautes Energies du CNRS at Jussieu, Paris, and especially Boris Pioline and Olivier Babelon for their warm hospitality while part of this work was carried out. E.D. wishes to thank the Aspen Center for Physics, where some of this work was done. M.G. gratefully acknowledges the hospitality of the International Center for Theoretical Science at the Tata Institute, Mumbai and the Department of Physics and Astronomy, Johns Hopkins University during the course of this work. 


\section{A Lie algebras, real forms, coincidences at low rank}

In this section, we shall briefly review the definitions of the classical complex Lie algebras, their real forms, various useful intersection and inclusion relations, and we shall give a summary of their coincidences at low rank (see for example [81]). The starting point for the definitions of the ordinary Lie algebras is $S L(m, \mathbf{C})$, which consists of $m \times m$ traceless matrices with complex entries. We have the following complex Lie algebras,

$$
\begin{aligned}
S O(m, \mathbf{C}) & \equiv\left\{A \in S L(m, \mathbf{C}), A^{t}+A=0\right\} \\
S p(2 m, \mathbf{C}) & \equiv\left\{A \in S L(m, \mathbf{C}), A^{t} J_{2 m}+J_{2 m} A=0\right\}
\end{aligned}
$$

and their real forms are as follows,

$$
\begin{aligned}
S U(m, n) & \equiv\left\{A \in S L(m+n, \mathbf{C}), A^{\dagger} I_{m, n}+I_{m, n} A=0\right\} \\
S O(m, n) & \equiv\left\{A \in S L(m+n, \mathbf{R}), A^{t} I_{m, n}+I_{m, n} A=0\right\} \\
S p(2 m, 2 n) & \equiv S p(2 m+2 n, \mathbf{C}) \cap S U(2 m, 2 n) \\
S O\left(2 m^{*}\right) & \equiv\left\{A \in S O(2 m, \mathbf{C}), A^{\dagger} J_{2 m}+J_{2 m} A=0\right\} \\
S U\left(2 m^{*}\right) & \equiv\left\{A \in S L(2 m, \mathbf{C}), A J_{2 m}-J_{2 m} A^{*}=0\right\}
\end{aligned}
$$

The matrices $I_{m, n}$ and $J_{2 m}$ are defined by,

$$
I_{m, n}=\left(\begin{array}{cc}
I_{m} & 0 \\
0 & -I_{n}
\end{array}\right) \quad J_{2 m}=\left(\begin{array}{cc}
0 & I_{m} \\
-I_{m} & 0
\end{array}\right)
$$

and $I_{m}$ is the identity matrix in dimension $m$. The Lie algebras $S U(m)=S U(m, 0)$, $S O(m)=S O(m, 0), S O\left(2^{*}\right)=S O(2)$, and $S p(2 m)=S p(2 m, 0)$ correspond to compact Lie groups; all other cases correspond to non-compact Lie groups.

\section{A.1 Intersections and inclusions}

It will be useful to have the following intersection and inclusion relations at our disposal,

$$
\begin{aligned}
S O(2 n) \cap S p(2 n) & =U(n) \\
S O\left(2 n^{*}\right) \cap U(2 n) & =U(n) \\
S p(2 n, \mathbf{R}) \cap U(2 n) & =U(n)
\end{aligned}
$$




\section{A.2 Coincidences at low rank}

Coincidences at low rank of the complex Lie algebras are as follows,

$$
\begin{aligned}
& S O(3, \mathbf{C})=S L(2, \mathbf{C})=S p(2, \mathbf{C}) \\
& S O(4, \mathbf{C})=S L(2, \mathbf{C}) \oplus S L(2, \mathbf{C}) \\
& S O(5, \mathbf{C})=S p(4, \mathbf{C}) \\
& S O(6, \mathbf{C})=S L(4, \mathbf{C})
\end{aligned}
$$

Coincidences at low rank of compact real forms are

$$
\begin{aligned}
& S O(3)=S U(2)=S p(2) \\
& S O(4)=S O(3) \oplus S O(3) \\
& S O(5)=S p(4) \\
& S O(6)=S U(4)
\end{aligned}
$$

Coincidences at low rank of non-compact real forms are,

$$
\begin{aligned}
S O(1,2) & =S U(1,1)=S p(2, \mathbf{R})=S L(2, \mathbf{R}) \\
S O\left(4^{*}\right) & =S O(1,2) \oplus S O(3) \\
S O(1,3) & =S L(2, \mathbf{C}) \\
S O(2,2) & =S O(1,2) \oplus S O(1,2) \\
S O(1,4) & =S p(2,2) \\
S O(2,3) & =S p(4, \mathbf{R}) \\
S O\left(6^{*}\right) & =S U(1,3) \\
S O(1,5) & =S U\left(4^{*}\right) \\
S O(2,4) & =S U(2,2) \\
S O(3,3) & =S L(4, \mathbf{R}) \\
S O\left(8^{*}\right) & =S O(2,6)
\end{aligned}
$$

Proofs of these equalities may be found in [81]. 


\section{B Non-exceptional embeddings}

A number of cases involve embeddings of real forms of the $S U$ type into real forms of the $O S p$ type, and vice versa, and are not simply settled by inspection of known subalgebra relations, or by simple arguments involving their maximal bosonic subalgebras. Some progress on the remaining cases may be made using Dynkin diagram techniques [99]. Inspection of the Dynkin diagrams allows one to recognize the regular subalgebras by deleting appropriate roots such as, for example, in the embedding $S L(m \mid n) \subset O S p(2 m \mid 2 n)$. Dynkin diagrams also allow one to obtain some of the singular subalgebras by using the "folding trick" such as, for example, in the embedding $O S p(2 m \mid 2 n) \subset S L(2 m \mid 2 n)$. These methods are, however, generally not sufficient for complete proofs when dealing with the real forms of the Lie superalgebras. In this section, we shall provide careful proofs of the embeddings of real forms of the $S U$ type into real forms of the $O S p$ type, and vice versa, using the matrix representations of these superalgebras of (4.13).

\section{B.1 Maximal $S U$ subalgebras of $O S p\left(2 m^{*} \mid 2 n\right)$}

We shall show the following maximal subalgebra relations,

$$
S U(p, q \mid n) \oplus U(1) \subset O S p\left(2 m^{*} \mid 2 n\right) \quad p+q=m, \quad p, q \geq 0
$$

We begin by recalling the definition of $M \in O S p\left(2 m^{*} \mid 2 n\right)$ :

$$
\begin{array}{rlrl}
M^{s t} K+K M & =0 & K & =\left(\begin{array}{cc}
I_{2 m} & 0 \\
0 & J_{2 n}
\end{array}\right) \\
\left(M^{*}\right)^{s t} \tilde{K}+\tilde{K} M & =0 & \tilde{K} & =\left(\begin{array}{cc}
J_{2 m} & 0 \\
0 & I_{2 n}
\end{array}\right)
\end{array}
$$

The presence of a $U(1)$ factor guarantees that the $S U(p, q \mid n)$ Lie superalgebra can be realized as the invariance algebra of a bosonic element $T$ in $O S p\left(2 m^{*} \mid 2 n\right)$. The embedding of the $S U(n)$ subalgebra of $S U(p, q \mid n)$ into the $S p(2 n)$ subalgebra of $O S p\left(2 m^{*} \mid 2 n\right)$ is unique, and is produced as the invariance subalgebra of the generator $J_{2 n}$. The $S U(p, q)$ subalgebra of $S O\left(2 m^{*}\right)$ will be obtained as the invariance subalgebra of an as yet undetermined generator $T_{1} \in S O\left(2 m^{*}\right)$. Thus, the $S U(p, q \mid n)$ is specified by,

$$
[T, M]=0 \quad T=\left(\begin{array}{cc}
T_{1} & 0 \\
0 & J_{2 n}
\end{array}\right)
$$

We shall now search for a matrix representation of this $S U(p, q \mid n)$ by investigating the simultaneous solution to the conditions of (B.2) and (B.3) on $M$. To do so, we decompose 
$M$ into blocks,

$$
M=\left(\begin{array}{cc}
A & B \\
C & D
\end{array}\right)
$$

where, as usual, $A, B, C, D$ are matrices respectively of dimension $2 m \times 2 m, 2 m \times 2 n, 2 n \times 2 m$, and $2 n \times 2 n$. In terms of the blocks $A, B, C, D$, the first condition of (B.2) becomes,

$$
\begin{aligned}
A^{t}+A & =0 \\
D^{t} J_{2 n}+J_{2 n} D & =0
\end{aligned} \quad B=-C^{t} J_{2 n}
$$

It will be convenient to recast the second condition of (B.2) by using the first condition of (B.2) to eliminate $M^{\text {st }}$ in favor of $M$. The combined condition then takes the form $M^{*}=K^{-1} \tilde{K} M \tilde{K}^{-1} K$, and decomposes as follows into the block components of $M$,

$$
\begin{array}{ll}
A^{*}=-J_{2 m} A J_{2 m} & B^{*}=+J_{2 m} B J_{2 n} \\
D^{*}=-J_{2 n} D J_{2 n} & C^{*}=+J_{2 n} C J_{2 m}
\end{array}
$$

Finally, the condition (B.3) becomes,

$$
\begin{array}{rlrl}
{\left[T_{1}, A\right]} & =0 & B J_{2 n}-T_{1} B & =0 \\
{\left[J_{2 n}, D\right]} & =0 & J_{2 n} C-C T_{1} & =0
\end{array}
$$

The combined conditions on the matrix $D$ imply that it is indeed an element of $S U(n) \oplus U(1)$.

\section{B.1.1 Solving for the fermionic generators}

Next, we analyze the conditions on the fermionic generators, represented by the matrices $B$ and $C$. Eliminating $B$ in terms of $C$, using equation $B=-C^{t} J_{2 n}$ of (B.2), gives $C^{*}=J_{2 n} C J_{2 m}$ (which is identical to the third equation in (B.3)), and $J_{2 n} C=-C T_{1}^{t}$. The remaining independent equations for $C$ are thus,

$$
\begin{aligned}
C^{*} & =J_{2 n} C J_{2 m} \\
J_{2 n} C & =+C T_{1} \\
J_{2 n} C & =-C T_{1}^{t}
\end{aligned}
$$

In any embedding of $S U(p, q \mid n)$ into $O S p\left(2 m^{*} \mid 2 n\right)$ with $p+q=m$, the number of fermionic generators of $S U(p, q \mid n)$ is fixed to be $2 m n$ real generators, out of the $4 m n$ real generators of $O S p\left(2 m^{*} \mid 2 n\right)$, thus preserving half thereof. As a generator of the algebra $O S p\left(2 m^{*} \mid 2 n\right)$, 
the matrix $C$ has $4 m n$ real independent generators, so that the second and third equations of (B.8) must be responsible for precisely halving that number down to $2 m n$. This condition forces the matrix $T_{1}$ to be of maximum rank $2 m$, and the matrix $C$ to have no non-trivial null space. The relations of (B.8) then imply further restrictions on $T_{1}$, which may be obtained as follows. Eliminating $J_{2 n} C$ between the first and second equations, we get $C^{*}=C T_{1} J_{2 m}$. Iterating this equation, as well as the middle equation above, eliminating $J_{2 n} C$ between the last two equations, and using the fact that $C$ has no non-trivial null space, we derive the following requirements on $T_{1}$,

$$
\begin{aligned}
\left(T_{1}\right)^{2} & =-I_{2 m} \\
T_{1} J_{2 m} T_{1}^{*} J_{2 m} & =+I_{2 m} \\
T_{1}^{t} & =-T_{1}
\end{aligned}
$$

Note that the second condition is equivalent to $J_{2 m} T_{1}^{*}=T_{1} J_{2 m}$, upon using the first condition. Any such matrix $T_{1}$ will support $2 m n$ real fermionic generators.

\section{B.1.2 Solving for the $S U(p, q)$ subalgebra of $S O\left(2 m^{*}\right)$}

The remaining conditions on the matrix $A$, with $T_{1}$ satisfying (B.9), are given by,

$$
\begin{aligned}
A^{t}+A & =0 \\
J_{2 m} A^{*}+A J_{2 m} & =0 \\
T_{1} A-A T_{1} & =0
\end{aligned} \quad J_{2 m}=\left(\begin{array}{cc}
0 & I_{m} \\
-I_{m} & 0
\end{array}\right)
$$

It is standard to solve the first two conditions above combined (see for example [81], p. 446), in terms of $m \times m$ matrices $Z_{1}$ and $Z_{2}$,

$$
A=\left(\begin{array}{cc}
Z_{1} & Z_{2} \\
-Z_{2}^{*} & Z_{1}^{*}
\end{array}\right) \quad\left\{\begin{array}{c}
Z_{1}^{t}=-Z_{1} \\
Z_{2}^{t}=Z_{2}^{*}
\end{array}\right.
$$

It is actually more convenient to decompose $Z_{1}$ and $Z_{2}$ into real matrices $X_{1}, Y_{1}, X_{2}$, and $Y_{2}$,

$$
\begin{array}{llr}
Z_{1}=X_{1}+i Y_{1} & X_{1}^{t}=-X_{1} & Y_{1}^{t}=-Y_{1} \\
Z_{2}=X_{2}+i Y_{2} & X_{2}^{t}=+X_{2} & Y_{2}^{t}=-Y_{2}
\end{array}
$$

so that we have

$$
A=\left(\begin{array}{cc}
X_{1} & X_{2} \\
-X_{2} & X_{1}
\end{array}\right)+\left(\begin{array}{cc}
i Y_{1} & i Y_{2} \\
i Y_{2} & -i Y_{1}
\end{array}\right)
$$


The first term on the right hand side is anti-hermitean, while the second is hermitean, corresponding respectively to the compact and non-compact directions of Lie algebra. Setting $Y_{1}=Y_{2}=0$ gives the $U(m)$ subalgebra of $S O\left(2 m^{*}\right)$, which may be realized by demanding that $A$ commute with $T_{1}=J_{2 m}$. This value for $T_{1}$ satisfies all three conditions (B.9), thereby providing a proof of (B.1) for the special case where $p q=0$, namely $S U(m \mid n) \subset$ $O S p\left(2 m^{*} \mid 2 n\right)$. Note that, in view of the remarks on the (in)equivalence of real forms of section 4.5, the basis in which $T_{1}$ takes the form $J_{2 m}$ must coincide with the basis used for the matrix $J_{2 m}$ of (B.10).

To prove the subalgebra relations (B.1) for $p q \neq 0$, we concentrate first on the compact (i.e. anti-hermitean) part of the maximal bosonic subalgebra. The embedding $S U(p, q \mid n) \oplus$ $U(1) \subset O S p\left(2 m^{*} \mid 2 n\right)$ with $p+q=m$, requires the compact subalgebra $S U(p) \oplus S U(q) \oplus U(1)$ to be a subalgebra of the compact subalgebra $S U(m)$ on $S O\left(2 m^{*}\right)$, whose existence was established in the preceding paragraph, and whose matrix form corresponds to $Y_{1}=Y_{2}=0$ in (B.11). The subalgebra $S U(p) \oplus S U(q) \oplus U(1)$ may be realized inside $S U(m)$ as the invariance subalgebra of $T_{1}$ of the form

$$
T_{1}=\left(\begin{array}{cc}
r I_{p, q} & s I_{p, q} \\
-s I_{p, q} & r I_{p, q}
\end{array}\right) \quad I_{p, q}=\left(\begin{array}{cc}
I_{p} & 0 \\
0 & -I_{q}
\end{array}\right)
$$

for any pair of real numbers $r, s$, not both zero. To satisfy the first and the last conditions (B.9), we must have $r=0$, and $s= \pm 1$. The second condition of (B.9) is then automatic. This proves that the embedding (B.1) exists for all $p, q \geq 0$.

It is straightforward to exhibit the fermionic generator content. Parametrizing the $2 n \times$ $2 m$ matrix $C$, which satisfies the condition $C^{*}=J_{2 n} C J_{2 m}$ of (B.8), in terms of the block matrices $\alpha, \beta$ of dimension $n \times m$, gives the following explicit representation for $B$ and $C$,

$$
B=\left(\begin{array}{cc}
\beta^{\dagger} & -\alpha^{t} \\
-\alpha^{\dagger} & -\beta^{t}
\end{array}\right) \quad C=\left(\begin{array}{cc}
\alpha & \beta \\
\beta^{*} & -\alpha^{*}
\end{array}\right)
$$

The remaining conditions on $C$ of (B.8) restrict the blocks $\alpha, \beta$ as follows,

$$
\begin{aligned}
\alpha^{*} & =-\alpha I_{p, q} \\
\beta^{*} & =-\beta I_{p, q}
\end{aligned}
$$

The number of linearly independent generators adds up to $2 m n$, as required.

We may also exhibit the realization of the subalgebra $S U(p, q) \oplus U(1)$ inside $S U(m)$, by deriving an explicit parametrization of the solutions to (B.10) for the matrix $A$ in terms of $Z_{1}$ and $Z_{2}$ of (B.11). The requirement $\left[T_{1}, A\right]=0$ with $T_{1}$ given by (B.14) is solve by,

$$
Z_{1}=\left(\begin{array}{cc}
a_{1} & i b_{1} \\
-i b_{1}^{t} & c_{1}
\end{array}\right) \quad Z_{2}=\left(\begin{array}{cc}
a_{2} & i b_{2} \\
-i b_{2}^{t} & c_{2}
\end{array}\right)
$$


The dimensions of $a_{i}, b_{i}, c_{i}$, for $i=1,2$, are respectively $p \times p, p \times q$, and $q \times q$. The matrices $a_{i}, b_{i}, c_{i}$ are real; $a_{1}, d_{1}$ are anti-symmetric, while $a_{2}, d_{2}$ are symmetric. Assembling $Z_{1}$ and $Z_{2}$ into the matrix $A$ using (B.11), interchanging the second and third columns of $A$, and then interchanging also the second and third rows, gives the following expression,

$$
A \sim\left(\begin{array}{cccc}
a_{1} & a_{2} & i b_{1} & i b_{2} \\
-a_{2} & a_{1} & i b_{2} & -i b_{1} \\
-i b_{1}^{t} & -i b_{2}^{t} & d_{1} & d_{2} \\
-i b_{2}^{t} & i b_{1}^{t} & -d_{2} & d_{1}
\end{array}\right) \sim\left(\begin{array}{ccc}
a_{1}+i a_{2} & b_{1}-i b_{2} \\
b_{1}^{t}+i b_{2}^{t} & c_{1}+i c_{2}
\end{array}\right)
$$

It is immediate that the generators $a_{1}+i a_{2}$ and $c_{1}+i c_{2}$ are anti-hermitean and correspond to the $S U(p) \oplus S U(q) \oplus U(1)$ subalgebra of $S U(p, q) \oplus U(1)$, while $b_{1}-i b_{2}$ and $b_{1}^{t}+i b_{2}^{t}$ correspond to its non-compact directions.

\section{B.2 Maximal $S U$ subalgebras of $O S p(2 m \mid 2 n, \mathbf{R})$}

We shall show the following maximal embedding relations,

$$
S U(m \mid p, q) \oplus U(1) \subset O S p(2 m \mid 2 n, \mathbf{R}) \quad p+q=n, \quad p, q \geq 0
$$

We begin by recalling the definition of $M \in O S p(2 m \mid 2 n, \mathbf{R})$; we have $M^{*}=M$ and

$$
M^{s t} K+K M=0 \quad K=\left(\begin{array}{cc}
I_{2 m} & 0 \\
0 & J_{2 n}
\end{array}\right)
$$

The presence of a $U(1)$ factor guarantees that the $S U(m \mid p, q)$ Lie superalgebra can be realized as the invariance algebra of a bosonic element $T$ in $O S p(2 m \mid 2 n, \mathbf{R})$. The embedding of the $S U(m)$ subalgebra of $S U(m \mid p, q)$ into the $S O(2 m)$ subalgebra of $O S p(2 m \mid 2 n, \mathbf{R})$ is unique, and is produced as the invariance subalgebra of the generator $J_{2 m}$. The $S U(p, q)$ subalgebra of $S p(2 n)$ is obtained as the subalgebra of an as yet undetermined generator $T_{2} \in S p(2 n, \mathbf{R})$. Thus, the $S U(m \mid p, q)$ Lie superalgebra is specified by,

$$
[T, M]=0 \quad T=\left(\begin{array}{cc}
J_{2 m} & 0 \\
0 & T_{2}
\end{array}\right)
$$

We shall now seek a matrix representation by investigating the three simultaneous conditions of $M^{*}=M,(\mathrm{~B} .20)$ and (B.21). To do so, we decompose $M$ into blocks,

$$
M=\left(\begin{array}{cc}
A & B \\
C & D
\end{array}\right)
$$


with $A, B, C, D$ real matrices of respective dimensions $2 m \times 2 m, 2 m \times 2 n, 2 n \times 2 m$, and $2 n \times 2 n$. Condition (B.20) requires that

$$
\begin{aligned}
A^{t}+A & =0 \\
D^{t} J_{2 n}+J_{2 n} D & =0
\end{aligned} \quad B=-C^{t} J_{2 n}
$$

Condition (B.21) amounts to

$$
\begin{array}{rlrl}
{\left[J_{2 m}, A\right]} & =0 & B T_{2}-J_{2 m} B & =0 \\
{\left[T_{2}, D\right]} & =0 & C J_{2 m}-T_{2} C & =0
\end{array}
$$

The combined conditions on the matrix $A$ imply that $A$ is indeed an element of $S U(m) \oplus U(1)$.

\section{B.2.1 Solving for the fermionic generators}

Next, we analyze the conditions on the matrices $B$ and $C$. Eliminating $B$ in terms of $C$, using $B=-C^{t} J_{2 n}$ of the last line of (B.23), gives two equations for $C$,

$$
\begin{aligned}
C J_{2 m} & =T_{2} C \\
C & =-J_{2 n} T_{2}^{t} J_{2 n} C J_{2 m} \\
& =-J_{2 n} T_{2}^{t} J_{2 n} T_{2} C
\end{aligned}
$$

To have the correct number $2 m n$ of real fermionic generators for the superalgebra $S U(m \mid p, q)$ with $p+q=n$, the matrix $T_{2}$ must have maximal rank. Iterating the first equation in (B.25), and using also the last one, we retain the following conditions on $T_{2}$,

$$
\begin{aligned}
\left(T_{2}\right)^{2} & =-I_{2 n} \\
T_{2}^{t} & =J_{2 n} T_{2} J_{2 n}
\end{aligned}
$$

The last condition ensures that $T_{2}$ is of the form of the matrix $D$, so that $T \in O S p(2 m \mid 2 n, \mathbf{R})$.

\section{B.2.2 Solving for the $S U(p, q)$ subalgebra of $S p(2 n, \mathbf{R})$}

The remaining conditions on the real matrix $D$ are as follows,

$$
\begin{aligned}
D^{t} J_{2 n}+J_{2 n} D & =0 \\
{\left[T_{2}, D\right] } & =0
\end{aligned}
$$

It is standard to solve the first condition in terms of real $m \times m$ matrices $a, b, c$, so that

$$
D=\left(\begin{array}{cc}
a & b \\
c & -a^{t}
\end{array}\right)
$$


with $b^{t}=b$ and $c^{t}=c$, and no symmetry restrictions on $a$.

Choosing $T_{2}=J_{2 n}$, and enforcing the second condition in (B.27), gives the further restriction $a^{t}=-a$ and $c=-b$. The matrix $D$ is now real and anti-symmetric and, using a standard change of basis, the combination $a+i b$ then parametrizes the compact subalgebra $S U(n) \oplus U(1)$. This value of $T_{2}$ satisfies the conditions of (B.26), thereby providing a proof of (B.19) for the special case where $p q=0$, namely $S U(m \mid p, q) \subset O S p(2 m \mid 2 n, \mathbf{R})$.

To prove the subalgebra relations (B.19) for general $p, q$, we use the matrix,

$$
T_{2}=\left(\begin{array}{cc}
0 & I_{p, q} \\
-I_{p, q} & 0
\end{array}\right)
$$

$T_{2}$ clearly satisfies conditions (B.26), and thus will support $2 m n$ real fermionic generators needed for the $S U(m \mid p, p)$ subalgebra. It remains to check that $T_{2}$ indeed gives the correct real form $S U(p, q)$. The condition $\left[T_{2}, D\right]=0$ of (B.27) imposes the following relations between $a, b, c$ of (B.28),

$$
\begin{aligned}
a I_{p, q}+a^{t} I_{p, q} & =0 \\
b I_{p, q}+I_{p, q} c & =0
\end{aligned}
$$

whose general solution may be parametrized by the real block matrices $a_{1}, a_{2}, a_{3}, b_{1}, b_{2}, b_{3}$,

$$
a=\left(\begin{array}{ll}
a_{1} & a_{2} \\
a_{2}^{t} & a_{3}
\end{array}\right) \quad b=\left(\begin{array}{ll}
b_{1} & b_{2} \\
b_{2}^{t} & b_{3}
\end{array}\right)
$$

whose dimensions are $p \times p$ for $a_{1}, b_{1}, p \times q$ for $a_{2}, b_{2}$, and $q \times q$ for $a_{3}, b_{3}$, with $a_{1}, a_{3}$ antisymmetric, and $b_{1}, b_{3}$ symmetric. Assembling these expressions into the full matrix $D$ of (B.28), interchanging the second and third rows of this matrix $D$, and then its second and third columns, we obtain the following representation,

$$
D \sim\left(\begin{array}{cccc}
a_{1} & b_{1} & a_{2} & b_{2} \\
-b_{1} & a_{1} & b_{2} & -a_{2} \\
a_{2}^{t} & b_{2}^{t} & a_{3} & b_{3} \\
b_{2}^{t} & -a_{2}^{t} & -b_{3} & a_{3}
\end{array}\right) \sim\left(\begin{array}{cc}
a_{1}+i b_{1} & a_{2}+i b_{2} \\
a_{2}^{t}-i b_{2}^{t} & a_{3}+i b_{3}
\end{array}\right)
$$

It is immediate that the anti-hermitean generators $a_{1}+i b_{1}$ and $a_{3}+i b_{3}$ produce the compact subalgebra $S U(p) \oplus S U(q) \oplus U(1)$ of $S U(p, q) \oplus U(1)$, while the hermitean generators $a_{2}+i b_{2}$ and $a_{2}^{t}-i b_{2}^{t}$ produce its non-compact part. 


\section{B.3 The embedding $O S p(m \mid 2 n, \mathbf{R}) \subset S U(m \mid n, n)$}

Embedding of the compact part of the maximal bosonic subalgebras, $S O(m) \subset S U(m)$, is standard, while that of the non-compact part requires $S p(2 n, \mathbf{R}) \in S U(n, n)$. To realize the latter embedding explicitly, we use the following change of basis,

$$
J_{2 n}=\left(\begin{array}{cc}
0 & I_{n} \\
-I_{n} & 0
\end{array}\right)=-i S I_{n, n} S^{-1} \quad I_{n, n}=\left(\begin{array}{cc}
I_{n} & 0 \\
0 & -I_{n}
\end{array}\right)
$$

A convenient choice for $S$ is given by

$$
S=\frac{1}{\sqrt{2}}\left(\begin{array}{cc}
I_{n} & -i I_{n} \\
-i I_{n} & I_{n}
\end{array}\right) \quad S^{*}=S^{-1}
$$

By definition, any matrix $D \in S p(2 n, \mathbf{R})$ satisfies $D^{*}=D$, and $D^{t} J_{2 n}+J_{2 n} D=0$, from which it follows that $\operatorname{tr}(D)=0$. Using the above change of basis for $J_{2 n}$, we have equivalently, $D^{t} S I_{n, n} S^{-1}+S I_{n, n} S^{-1} D=0$. Setting $D_{s}=S^{-1} D S$ then implies,

$$
D_{s}^{\dagger} I_{n, n}+I_{n, n} D_{s}=0 \quad \operatorname{tr}\left(D_{s}\right)=0
$$

which is the defining relation for $D_{s} \in S U(n, n)$.

By definition, any matrix $M \in O S p(m \mid 2 n, \mathbf{R})$ satisfies $M^{*}=M$ and $M^{s t} K+K M=0$, from which it follows that $\operatorname{str}(M)=0$. The matrix $K=K_{m \mid 2 n}$ was defined in (4.11) and may be recast as follows, using the change of basis (B.33),

$$
K=\left(\begin{array}{cc}
I_{m} & 0 \\
0 & J_{2 n}
\end{array}\right)=S_{1} L S_{1}^{-1} \quad S_{1}=\left(\begin{array}{cc}
I_{m} & 0 \\
0 & S
\end{array}\right)
$$

The resulting matrix $L$ is given by

$$
L=\left(\begin{array}{cc}
I_{m} & 0 \\
0 & -i I_{n, n}
\end{array}\right)
$$

and coincides with the matrix $L_{m \mid n, n}$, defined in (4.11). Using the above change of basis, the orthosymplectic condition on $M$ becomes $M^{s t} S_{1} L S_{1}^{-1}+S_{1} L S_{1}^{-1} M=0$. Setting $M_{s}=$ $S_{1}^{-1} M S_{1}$ and using the fact that $\left(M_{s}^{*}\right)^{s t}=S_{1}^{-1} M^{s t} S_{1}$, implies that

$$
\left(M_{s}^{*}\right)^{s t} L+L M_{s}=0 \quad \operatorname{str}\left(M_{s}\right)=0
$$

which is the defining relation for $M_{s} \in S U(m \mid n, n)$. This concludes the proof. 


\section{B.4 The embedding $O S p\left(2 m^{*} \mid 2 n\right) \subset S U(m, m \mid 2 n)$}

The embedding of the compact part of the maximal bosonic subalgebras, $S p(2 n) \subset S U(2 n)$, holds by definition of $S p(2 n)$, while that of the non-compact part requires $S O\left(2 m^{*}\right) \subset$ $S U(m, m)$. The latter is realized explicitly via a change of basis of (B.33). By definition, any matrix $A \in S O\left(2 m^{*}\right)$ satisfies $A^{t}+A=0$ and $A^{\dagger} J_{2 m}+J_{2 m} A=0$, from which it follows that $\operatorname{tr}(A)=0$. Performing the change of basis given by $J_{2 m}=-i S I_{n, n} S^{-1}$, and defining $A_{s}=S^{-1} A S$ then implies,

$$
A_{s}^{\dagger} I_{n, n}+I_{n, n} A_{s}=0 \quad \operatorname{tr}\left(A_{s}\right)=0
$$

which are the defining relations of $A_{s} \in S U(m, m)$.

By definition, a matrix $M \in O S p\left(2 m^{*} \mid 2 n\right)$ satisfies $M=-K^{-1} M^{s t} K=-\tilde{K}^{-1}\left(M^{*}\right)^{s t} \tilde{K}$ from which it follows that $\operatorname{str}(M)=0$. The matrices $K=K_{2 m \mid 2 n}$ and $\tilde{K}=\tilde{K}_{2 m \mid 2 n}$ were defined in (4.11), and may be recast as follows, using the change of basis (B.33),

$$
\begin{array}{ll}
K=\left(\begin{array}{cc}
I_{m} & 0 \\
0 & J_{2 n}
\end{array}\right)=S_{2} K S_{2}^{-1} & S_{2}=\left(\begin{array}{cc}
S & 0 \\
0 & I_{2 n}
\end{array}\right) \\
\tilde{K}=\left(\begin{array}{cc}
J_{2 m} & 0 \\
0 & I_{2 n}
\end{array}\right)=S_{2} L S_{2}^{-1} & L=\left(\begin{array}{cc}
-i I_{m, m} & 0 \\
0 & I_{2 n}
\end{array}\right)
\end{array}
$$

In the new basis, the relation involving $\tilde{K}$ becomes

$$
S_{2} L S_{2}^{-1} M+\left(M^{*}\right)^{s t} S_{2} L S_{2}^{-1}=0
$$

In terms of $M_{s}=S_{2}^{-1} M S_{2}$ this implies

$$
\left(M_{s}^{*}\right)^{s t} L+L M_{s}=0 \quad \operatorname{str}\left(M_{s}\right)=0
$$

which is the defining relation of $M_{s} \in S U(m, m \mid 2 n)$. This concludes the proof. 


\section{Classification of exceptional sub-superalgebras}

In this appendix, we shall show that none of the exceptional simple basic Lie superalgebras $F(4), G(3)$, or $D(2,1 ; c)$ with $c \neq 1,-2,-1 / 2$, or any of their real forms, is a subalgebra of either the complex superalgebras $O S p(8 \mid 4)$ and $P S L(4 \mid 4)$, or of their real forms $O S p\left(8^{*} \mid 4\right)$, $O S p(8 \mid 4, \mathbf{R})$ or $\operatorname{PSU}(2,2 \mid 4)$.

Dealing with the exceptional superalgebras, just as dealing with the exceptional Lie algebras, is most easily done with the help of the roots in the Cartan formalism. Therefore, we shall first give a brief review of the root systems of the algebras $S L(m \mid n), O S p(2 m \mid 2 n)$, and the exceptional superalgebras $F(4), G(3)$ and $D(2,1 ; c)$.

\section{C.1 Root systems of the basic classical superalgebras}

Given a superalgebra $\mathcal{G}$, its maximal bosonic subalgebra will be denoted $\mathcal{G}_{\overline{0}}$, and its fermionic subspace by $\mathcal{G}_{\overline{1}}$. The algebra $\mathcal{G}$ is classical if $\mathcal{G}$ is simple, and if the representation of $\mathcal{G}_{\overline{0}}$ on $\mathcal{G}_{\overline{1}}$ is completely reducible. The sets of all bosonic and fermionic roots will be denoted by $\Delta_{\overline{0}}$ and $\Delta_{\overline{1}}$, respectively, and the set of all roots is then $\Delta=\Delta_{\overline{0}} \cup \Delta_{\overline{1}}$. For the superalgebras relevant here, they are given as follows,

\begin{tabular}{|c|c|c|c|}
\hline Superalgebra & $\overline{\Delta_{\overline{0}}}$ & $\overline{\Delta_{\overline{1}}}$ & normalizations \\
\hline$S L(m \mid n)$ & $\begin{array}{ll}\varepsilon_{i}-\varepsilon_{j}, & 1 \leq i \neq j \leq m \\
\delta_{a}-\delta_{b}, & 1 \leq a \neq b \leq n\end{array}$ & $\pm\left(\varepsilon_{i}-\delta_{a}\right)$ & $\begin{array}{l}\varepsilon_{i} \cdot \varepsilon_{j}=+\delta_{i j} \\
\delta_{a} \cdot \delta_{b}=-\delta_{a b}\end{array}$ \\
\hline$O S p(2 m \mid 2 n)$ & $\begin{array}{c} \pm \varepsilon_{i} \pm \varepsilon_{j}, \quad 1 \leq i \neq j \leq m \\
\pm \delta_{a} \pm \delta_{b}, \pm 2 \delta_{a}, 1 \leq a \neq b \leq n\end{array}$ & $\pm \varepsilon_{i} \pm \delta_{a}$ & $\begin{array}{l}\varepsilon_{i} \cdot \varepsilon_{j}=+\delta_{i j} \\
\delta_{a} \cdot \delta_{b}=-\delta_{a b}\end{array}$ \\
\hline$O S p(2 m+1 \mid 2 n)$ & $\begin{array}{l} \pm \varepsilon_{i} \pm \varepsilon_{j}, \pm \varepsilon_{i}, \quad 1 \leq i \neq j \leq m \\
\pm \delta_{a} \pm \delta_{b}, \pm 2 \delta_{a}, 1 \leq a \neq b \leq n\end{array}$ & $\pm \varepsilon_{i} \pm \delta_{a}, \quad \pm \delta_{a}$ & $\begin{array}{l}\varepsilon_{i} \cdot \varepsilon_{j}=+\delta_{i j} \\
\delta_{a} \cdot \delta_{b}=-\delta_{a b}\end{array}$ \\
\hline$F(4)$ & $\begin{array}{c} \pm \varepsilon_{i} \pm \varepsilon_{j}, \quad \pm \varepsilon_{i}, \quad 1 \leq i \neq j \leq 3 \\
\pm \delta\end{array}$ & $\frac{1}{2}\left( \pm \varepsilon_{1} \pm \varepsilon_{2} \pm \varepsilon_{3} \pm \delta\right)$ & $\begin{array}{c}\varepsilon_{i} \cdot \varepsilon_{j}=+\delta_{i j} \\
\delta^{2}=-3\end{array}$ \\
\hline$G(3)$ & $\begin{array}{c}\varepsilon_{i}-\varepsilon_{j}, \pm \varepsilon_{i}, \quad 1 \leq i \neq j \leq 3 \\
\pm 2 \delta\end{array}$ & $\pm \varepsilon_{i} \pm \pm \delta, \pm \delta$ & $\begin{array}{c}\varepsilon_{i} \cdot \varepsilon_{j}=1-3 \delta_{i j} \\
\varepsilon_{1}+\varepsilon_{2}+\varepsilon_{3}=0 \\
\delta^{2}=2\end{array}$ \\
\hline$D(2,1 ; c)$ & $i=1,2,3$ & $\pm \varepsilon_{1} \pm \varepsilon_{2} \pm \varepsilon_{3}$ & $\begin{array}{c}\varepsilon_{i} \cdot \varepsilon_{j}=0, i \neq j \\
2 \varepsilon_{1}^{2}=-1-\alpha \\
2 \varepsilon_{2}^{2}=1,2 \varepsilon_{3}^{2}=\alpha\end{array}$ \\
\hline
\end{tabular}

Table 19: Root systems of the basic classical Lie superalgebras.

The vectors $\varepsilon_{i}$ and $\delta_{a}$ form a basis for the space of roots. For all cases, we have $\varepsilon \cdot \delta=0$. 
Notice that, as a result, the inner product on the vector spaces generated by $\varepsilon, \delta$ is in each case of indefinite signature. In particular, notice that for $D(2,1 ; c)$, we have $\varepsilon_{1}^{2}+\varepsilon_{2}^{2}+\varepsilon_{3}^{2}=0$.

Because of the indefinite signature inner product, the choice of a simple root system is not unique, unlike in the case of ordinary Lie algebras. As a result, a given basic classical Lie superalgebra can be described by different Dynkin diagrams.

\section{C.2 Strategy}

To show that $\mathcal{H}=F(4), G(3)$, and $D(2,1 ; c)$ for $c \neq 1,-2,-1 / 2$, are not subalgebras of $\mathcal{G}=O S p(8 \mid 4), P S L(4 \mid 4)$ or of the real forms $O S p\left(8^{*} \mid 4\right), O S p(8 \mid 4, \mathbf{R})$, or $P S U(2,2 \mid 4)$, we shall use the strategy below. The maximal bosonic subalgebras will be denoted $\mathcal{H}_{\overline{0}}$ and $\mathcal{G}_{\overline{0}}$, while their fermionic counterparts will be denoted by $\mathcal{H}_{\overline{1}}$ and $\mathcal{G}_{\overline{1}}$

1. Exhibit the finite number of possible embeddings of $\mathcal{H}_{\overline{0}}$ into $\mathcal{G}_{\overline{0}}$. If we have $\mathcal{H}_{\overline{0}} \not \subset \mathcal{G}_{\overline{0}}$, then it follows that $\mathcal{H}$ is not a subalgebra of $\mathcal{G}$.

2. We now assume that $\mathcal{H}_{\overline{0}} \subset \mathcal{G}_{\overline{0}}$. Exhibit the finite number of possible embeddings of $\mathcal{H}_{1}$ into $\mathcal{G}_{\overline{1}}$, in the representation of $\mathcal{H}_{\overline{0}}$ on $\mathcal{H}_{\overline{1}}$ known on general grounds by the Lie superalgebra classification theorem. If no such embedding of $\mathcal{H}_{1}$ in $\mathcal{G}_{\overline{1}}$ exists, it follows that $\mathcal{H}$ is not a subalgebra of $\mathcal{G}$.

3. In the remaining cases where $\mathcal{H}_{\overline{0}} \subset \mathcal{G}_{\overline{0}}$, and $\mathcal{H}_{\overline{1}}$ can be embedded in $\mathcal{G}_{\overline{1}}$ in a suitable representation of $\mathcal{H}_{\overline{0}}$, one proceeds to compute the anti-commutators of $\mathcal{H}_{\overline{1}}$.

- If $\left\{\mathcal{H}_{\overline{1}}, \mathcal{H}_{\overline{1}}\right\} \subset \mathcal{H}_{\overline{0}}$, then $\mathcal{H}$ is a subalgebra of $\mathcal{G}$;

- If $\left\{\mathcal{H}_{\overline{1}}, \mathcal{H}_{\overline{1}}\right\} \not \subset \mathcal{H}_{\overline{0}}$, then $\mathcal{H}$ is not a subalgebra of $\mathcal{G}$;

\section{C.3 The superalgebra $P S U(2,2 \mid 4)$}

In the paragraphs below, we shall show that $P S U(2,2 \mid 4)$ does not have $F(4)$ and $G(3)$ as subalgebra, and that $D(2,1 ; c)$ is a subalgebra if and only if $c=1,-2,-1 / 2$.

\section{C.3.1 $F(4)$ is not a subalgebra of $P S U(2,2 \mid 4)$}

None of the maximal bosonic subalgebras $S L(2, \mathbf{R}) \oplus S O(p, 7-p)$ of the reals forms $F(4 ; p)$ for $p=0,1,2,3$ of $F(4)$ is a subalgebra of the maximal bosonic subalgebra $S U(2,2) \oplus S U(4)$ of $P S U(2,2 \mid 4)$, because $S O(7)$ is not a subalgebra of $S U(4)=S O(6)$, and $S O(p, 7-p)$ is not a subalgebra of $S U(2,2)=S O(2,4)$. Hence none of the real forms of $F(4)$ is not a subalgebra of $P S U(2,2 \mid 4)$. 


\section{C.3.2 $\quad G(3)$ is not a subalgebra of $P S U(2,2 \mid 4)$}

Neither one of the maximal bosonic subalgebras $S L(2, \mathbf{R}) \oplus G_{2,2 p}$, for $p=0,1$, of the reals forms $G(3 ; p)$ of $G(3)$ is a subalgebra of $P S U(2,2 \mid 4)$, since neither $G_{2,2 p}$ for $p=0,1$ is a subalgbera of $S U(2,2) \oplus S U(4)$. As a result, $G(3)$ is not a subalgebra of $P S U(2,2 \mid 4)$.

\section{C.3.3 $D(2,1 ; c)$ subalgebra of $P S U(2,2 \mid 4)$ implies $c=1,-2,-1 / 2$}

The case of $D(2,1 ; c)$ is more intricate. To investigate this case systematically, we shall follow the steps given in the Strategy section.

1. The maximal bosonic subalgebra of the real form $D(2,1 ; c ; p)$, for $p=0,1,2$, is given by $S L(2, \mathbf{R}) \oplus S O(p, 4-p)$. For $p=1,2$, this algebra is respectively $S O(1,2) \oplus S O(1,3)$, and $S O(1,2) \oplus S O(2,2)$, neither of which is a subalgebra of $S O(2,4) \oplus S O(6)$. This leaves only the case $p=0$, for which the algebra $\mathcal{S}=S U(1,1) \oplus S U(2) \oplus S U(2)$ is a subalgebra of $S U(2,2) \oplus S U(4)$, with $S U(1,1) \subset S U(2,2)$, and $S U(2) \oplus S U(2) \in S U(4)$. Several possible embedding exist for both.

2. The fermionic part of $D(2,1 ; c ; 0)$ must transform under $\mathcal{S}$ in the representation $(2,2,2)$. This requirement restricts the embedding of $S U(1,1)$ in $S U(2,2)$ to be such that the $\mathbf{4}$ of $S U(2,2)$ decomposes under $S U(1,1)$ as $\mathbf{4}=\mathbf{2}+\mathbf{1}+\mathbf{1}$. Similarly, the $\mathbf{4}$ of $S U(4)$ must decompose under $S U(2) \oplus S U(2)=S O(4)$ as $\mathbf{4}=(\mathbf{2}, \mathbf{2})$, the vector representation of $S O(4)$. Thus, the embedding of $\mathcal{S}$ into $S U(2,2) \oplus S U(4)$, is unique and may be realized as follows in terms of the root generators of $P S U(2,2 \mid 4)$,

$$
\mathcal{S}\left\{\begin{array}{c}
E_{ \pm\left(\delta_{2}-\delta_{3}\right)} \\
E_{ \pm\left(\varepsilon_{1}-\varepsilon_{2}\right)}+E_{ \pm\left(\varepsilon_{3}-\varepsilon_{4}\right)} \\
E_{ \pm\left(\varepsilon_{1}-\varepsilon_{3}\right)}+E_{ \pm\left(\varepsilon_{2}-\varepsilon_{4}\right)}
\end{array}\right\} \quad \begin{aligned}
& S L(2) \\
& S U(2) \\
& \\
& S U(2)
\end{aligned}
$$

The 32 fermionic generators of $\operatorname{PSU}(2,2 \mid 4)$, namely $E_{ \pm\left(\varepsilon_{i}-\delta_{a}\right)}$ with $i, a=1,2,3$, 4 , transform under $\mathcal{S}$ in a reducible representation. The 16 generators $\left\{E_{ \pm\left(\delta_{1}-\varepsilon_{i}\right)}, E_{ \pm\left(\delta_{4}-\varepsilon_{i}\right)}\right\}$ for $i=1,2,3,4$ are singlets under $S L(2)$, and cannot belong to $D(2,1 ; c ; 0)_{\overline{1}}$. The remaining 16 generators $\left\{E_{ \pm\left(\delta_{2}-\varepsilon_{i}\right)}, E_{ \pm\left(\delta_{3}-\varepsilon_{i}\right)}\right\}$ for $i=1,2,3,4$ transform non-trivially under $\mathcal{S}$, in a reducible representation. This is established by noticing that the involution $\rho$, defined by,

$$
\rho\left\{\begin{array} { l l l } 
{ \delta _ { 1 } } & { \leftrightarrow } & { - \delta _ { 4 } } \\
{ \delta _ { 2 } } & { \leftrightarrow } & { - \delta _ { 3 } }
\end{array} \quad \rho \left\{\begin{array}{lll}
\varepsilon_{1} & \leftrightarrow & -\varepsilon_{4} \\
\varepsilon_{2} & \leftrightarrow & -\varepsilon_{3}
\end{array}\right.\right.
$$

commutes with the generators of $\mathcal{S}$ in (C.1). Thus, the 16-dimensional representation of generators $\left\{E_{ \pm\left(\delta_{2}-\varepsilon_{i}\right)}, E_{ \pm\left(\delta_{3}-\varepsilon_{i}\right)}\right\}$ for $i=1,2,3,4$ may be decomposed according to the 
eigenspaces of $\rho$ associated with the eigenvalues $\sigma= \pm 1$,

$$
R_{\sigma}\left\{\begin{array}{l}
E_{ \pm\left(\varepsilon_{1}-\delta_{2}\right)}+\sigma E_{ \pm\left(\delta_{3}-\varepsilon_{4}\right)} \\
E_{ \pm\left(\varepsilon_{1}-\delta_{3}\right)}+\sigma E_{ \pm\left(\delta_{2}-\varepsilon_{4}\right)} \\
E_{ \pm\left(\varepsilon_{2}-\delta_{2}\right)}+\sigma E_{ \pm\left(\delta_{3}-\varepsilon_{3}\right)} \\
E_{ \pm\left(\varepsilon_{2}-\delta_{3}\right)}+\sigma E_{ \pm\left(\delta_{2}-\varepsilon_{3}\right)}
\end{array}\right\}
$$

Both spaces $R_{ \pm}$are 8-dimensional. The representation of $\mathcal{S}$ under which $R_{+}$and $R_{-}$transform is $(\mathbf{2}, \mathbf{2}, \mathbf{2})$. To form the superalgebra $D(2,1 ; c ; 0)$ from the bosonic subalgebra $\mathcal{S}$ we have two, and only two choices for the fermionic generators, namely $R_{+}$or $R_{-}$.

3. The structure relations of the fermionic generators are now easily worked out and are found to reproduce either those of $O S p\left(4^{*} \mid 2\right)=D(2,1 ; c ; 0)$, for $c=-2,-1 / 2$, or those of $O S p(4 \mid 2, \mathbf{R})=D(2,1 ; 1 ; 0)$ We note that the embedding of $O S p\left(4^{*} \mid 2\right)$ into $P S U(2,2 \mid 4)$ proceeds through the following chain,

$$
O S p\left(4^{*} \mid 2\right) \subset O S p\left(4^{*} \mid 4\right) \subset P S U(2,2 \mid 4)
$$

where the last embedding corresponds to the real form of $O S p(4 \mid 4) \subset P S L(4 \mid 4)$ of Table 8.

\section{C.4 The superalgebras $O S p\left(8^{*} \mid 4\right)$ and $O S p(8 \mid 4, \mathbf{R})$}

Next, we shall show that neither $F(4), G(3), D(2,1 ; c)$, nor any one of their real forms, are subalgebras of $O S p\left(8^{*} \mid 4\right)$ or $O S p(8 \mid 4, \mathbf{R})$. We recall the respective maximal bosonic subalgebras of these real forms of $O S p(8 \mid 4)$; they are given by

$$
\begin{array}{ll}
O S p\left(8^{*} \mid 4\right) & S O\left(8^{*}\right) \oplus S p(4)=S O(2,6) \oplus S O(5) \\
O S p(8 \mid 4, \mathbf{R}) & S O(8) \oplus S p(4, \mathbf{R})=S O(8) \oplus S O(2,3)
\end{array}
$$

We note that $S O(2,6)$ and $S p(4, \mathbf{R})$ are precisely the non-compact forms of respectively $S O(8)$ and $S p(4)$ which appear naturally when describing these real forms in the CartanWeyl basis. Using this basis will allow us to study both non-compact real forms $O S p\left(8^{*} \mid 4\right)$ and $\operatorname{OSp}(8 \mid 4, \mathbf{R})$ simultaneously.

The system of all roots of $O S p(8 \mid 4)$ is given by

$$
\begin{array}{lll}
\left( \pm \delta_{1} \pm \delta_{2}\right) & \left( \pm 2 \delta_{a}\right) & S p(4) \\
\left( \pm \varepsilon_{i} \pm \varepsilon_{j}\right) & i \neq j & S O(8) \\
\left( \pm \delta_{a} \pm \varepsilon_{i}\right) & & \text { fermionic }
\end{array}
$$

The root vector space has a non-degenerate indefinite inner product for which $\delta_{a} \cdot \varepsilon_{i}=0$, $\delta_{a} \cdot \delta_{b}=-\delta_{a b}$, and $\varepsilon_{i} \cdot \varepsilon_{j}=\delta_{i j}$, and we have $a, b=1,2$, and $i, j=1,2,3,4$. 


\section{C.4.1 $F(4)$ is not a subalgebra of $O S p\left(8^{*} \mid 4\right)$}

The maximal bosonic subalgebra of the real form $F(4 ; p)$ of $F(4)$ is $S O(1,2) \oplus S O(p, 7-p)$ for $p=0,1,2,3$. Clearly, we have $S O(1,2) \oplus S O(p, 7-p) \not \subset S O(2,6)$. When $p=0$, the component $S O(7)$ fits neither in $S O(2,6)$, nor in $S p(4)=S O(5)$. When $p \neq 0$, both components of $S O(1,2) \oplus S O(p, 7-p)$ are non-compact; since they do not simultaneoulsy fit into $S O(2,6)$, it follows that $F(4 ; p)$ is not a subalgebra of $O S p\left(8^{*} \mid 4\right)$ for $p=0,1,2,3$.

\section{C.4.2 $F(4)$ is not a subalgebra of $O S p(8 \mid 4, \mathbf{R})$}

When $p \neq 0$, the maximal bosonic algebra of $F(4 ; p)$, namely $S O(1,2) \oplus S O(p, 7-p)$, has two non-compact components, which do not simultaneously fit into $S p(4, \mathbf{R})$, so that $F(4 ; p)$ is not a subalgebra of $O S p(8 \mid 4, \mathbf{R})$ for $p=1,2,3$. The only case left is the real form $F(4 ; 0)$.

1. To realize the bosonic subalgebra $S p(2, \mathbf{R}) \oplus S O(7)$ of $F(4 ; 0)$, the $S O(7)$ part must be a subalgebra of $S O(8)$, and the $S p(2, \mathbf{R})$ part must be a subalgebra of $S p(4, \mathbf{R})$. There is a single embedding of $S O(7)$ into $S O(8)$ (up to the triality of $S O(8)$ ), which may be obtained by "folding" any two of the orthogonal simple roots of $S O(8)$, to be specific we fold the roots $\varepsilon_{3} \pm \varepsilon_{4}$. The resulting simple root $\beta$ of $S O(7)$ corresponds to the following linear combination of generators of $S O(8)$,

$$
E_{\beta} \sim E_{\varepsilon_{3}-\varepsilon_{4}}+E_{\varepsilon_{3}+\varepsilon_{4}}
$$

(Note that by this same "folding", one obtains the subalgebra $O S p(7 \mid 4)$ from $O S p(8 \mid 4)$.) Using also the remaining two simple root generators of $S O(7)$, namely $E_{\varepsilon_{1}-\varepsilon_{2}}$ and $E_{\varepsilon_{2}-\varepsilon_{3}}$, we deduce all 18 roots of $S O(7)$, expressed in the $S O(8)$ basis,

$$
\begin{array}{ll}
E_{ \pm \varepsilon_{1} \pm \varepsilon_{2}} & E_{\varepsilon_{3}-\varepsilon_{4}}+E_{\varepsilon_{3}+\varepsilon_{4}} \\
E_{ \pm \varepsilon_{2} \pm \varepsilon_{3}} & E_{\varepsilon_{1}-\varepsilon_{4}}+E_{\varepsilon_{1}+\varepsilon_{4}} \\
E_{ \pm \varepsilon_{1} \pm \varepsilon_{3}} & E_{\varepsilon_{2}-\varepsilon_{4}}+E_{\varepsilon_{2}+\varepsilon_{4}}
\end{array}
$$

where the \pm signs in the left column are all uncorrelated. To realize the full maximal bosonic subalgebra $S p(2, \mathbf{R}) \oplus S O(7)$ of $F(4 ; 0)$ as a subalgebra of $S O(8) \oplus S p(4, \mathbf{R})$, we

need to realize also $S p(2, \mathbf{R})$ as a subalgebra of $S p(4, \mathbf{R})$. There are 3 possible inequivalent embeddings of $S p(2, \mathbf{R})=S O(1,2)$ into $S p(4, \mathbf{R})=S O(2,3)$, corresponding to the following decompositions of the $\mathbf{5}$ of $S O(2,3)$, and $S p(4, \mathbf{R})$ respectively:
(a)
$5=2 \oplus 2 \oplus 1$
$\mathbf{4}=\mathbf{2} \oplus \mathbf{1} \oplus \mathbf{1}$
(c)
$5=3 \oplus 1 \oplus 1$
$4=2 \oplus 2$
$5=5$
$4=4$ 
The associated root generators of $S p(2, \mathbf{R})$ in the $S p(4, \mathbf{R})$ basis for these three cases are given as follows, (up to equivalences),

$$
\begin{array}{ll}
\text { (a) } & E_{2 \delta_{1}}, E_{-2 \delta_{1}} \\
& E_{2 \delta_{1}}+E_{2 \delta_{2}}, E_{-2 \delta_{1}}+E_{-2 \delta_{2}} \\
(b) & E_{\delta_{1}+\delta_{2}}, E_{-\delta_{1}-\delta_{2}}
\end{array}
$$

Thus, in total, the maximal bosonic subalgebra $S L(2) \times S O(7)$ of $F(4 ; 0)$ can be embedded in three inequivalent ways in the maximal bosonic subalgebra of $O S p(8 \mid 4, \mathbf{R})$, according to the three cases listed above.

2. The fermionic generators of $F(4 ; 0)$ must transform under the representation $\left(2,8_{s}\right)$ of $S p(2, \mathbf{R}) \times S O(7)$, where the $\mathbf{8}_{s}$ is the irreducible spinor representation of $S O(7)$. Since we have explicit expressions for the generators of $S O(7)$ in the $S O(8)$ Cartan basis, we may compute directly how the fermionic generators $E_{ \pm \varepsilon_{i} \pm \delta_{a}}$ transform under this $S O(7)$. For every $\delta \in\left\{ \pm \delta_{1}, \pm \delta_{2}\right\}$, we have

$$
\mathbf{7}\left\{\begin{array}{c}
E_{ \pm \varepsilon_{i}+\delta} \\
E_{\varepsilon_{4}+\delta}+E_{-\varepsilon_{4}+\delta}
\end{array} \quad i=1,2,3 \quad \mathbf{1}\left\{E_{\varepsilon_{4}+\delta}-E_{-\varepsilon_{4}+\delta}\right.\right.
$$

Hence, it is impossible to realize the 8-dimensional irreducible spinor representation of $S O(7)$. As a result, $F(4 ; 0)$ cannot be a subalgebra of $O S p(8 \mid 4, \mathbf{R})$, irrespective of the realization of the $\operatorname{Sp}(2, \mathbf{R})$ into $S p(4, \mathbf{R})$.

\section{C.4.3 $G(3)$ is not a subalgebra of $O S p\left(8^{*} \mid 4\right)$ or $O S p(8 \mid 4, \mathbf{R})$}

The maximal bosonic subalgebra $S L(2, \mathbf{R}) \oplus G_{2,2}$ of the real form $G(3 ; 1)$ cannot fit into either $S O(2,6) \oplus S p(4)$ or $S O(8) \oplus S p(4, \mathbf{R})$, and hence $G(3 ; 1)$ is not a subalgebra of either $O S p\left(8^{*} \mid 4\right)$ or $O S p(8 \mid 4, \mathbf{R})$. Also, the maximal bosonic subalgebra $S L(2, \mathbf{R}) \oplus G_{2}$ of the real form $G(3 ; 0)$, with $G_{2}$ compact, cannot fit into $S O(2,6) \oplus S p(4)$, and hence $G(3 ; 0)$ is not a subalgebra of $O S p\left(8^{*} \mid 4\right)$. The only case left is $G(3 ; 0)$ as a subalgebra of $O S p(8 \mid 4, \mathbf{R})$.

1. To realize the maximal bosonic subalgebra $S L(2, \mathbf{R}) \oplus G_{2}$ of the real form $G(3 ; 0)$ as a subalgebra of $S O(8) \oplus S p(4, \mathbf{R}), G_{2}$ must be in $S O(8)$ and $S L(2, \mathbf{R})$ must be in $S p(4 . \mathbf{R})$. There is a single embedding of $G_{2}$ into $S O(8)$, (up to the triality of $S O(8)$ ), realized as $G_{2} \subset S O(7) \subset S O(8)$, which may be obtained by "folding" all three orthogonal simple roots of $S O(8), \varepsilon_{1}-\varepsilon_{2}, \varepsilon_{3}-\varepsilon_{4}$, and $\varepsilon_{3}+\varepsilon_{4}$. The resulting simple root $\beta$ of $G_{2}$ corresponds to the following linear combination of generators of $S O(8)$,

$$
E_{\beta} \sim E_{\varepsilon_{1}-\varepsilon_{2}}+E_{\varepsilon_{3}-\varepsilon_{4}}+E_{\varepsilon_{3}+\varepsilon_{4}}
$$


Using the remaining simple root $E_{\varepsilon_{2}-\varepsilon_{3}}$ of $G_{2}$, we deduce the remaining root system of generators of $G_{2}$ in the $S O(8)$ basis of generators,

$$
\begin{array}{ll}
E_{ \pm\left(\varepsilon_{2}-\varepsilon_{3}\right)} & E_{ \pm\left(\varepsilon_{1}-\varepsilon_{2}\right)}+E_{ \pm\left(\varepsilon_{3}-\varepsilon_{4}\right)}+E_{ \pm\left(\varepsilon_{3}+\varepsilon_{4}\right)} \\
E_{ \pm\left(\varepsilon_{1}+\varepsilon_{2}\right)} & E_{ \pm\left(\varepsilon_{1}-\varepsilon_{3}\right)}+E_{ \pm\left(\varepsilon_{2}-\varepsilon_{4}\right)}+E_{ \pm\left(\varepsilon_{2}+\varepsilon_{4}\right)} \\
E_{ \pm\left(\varepsilon_{1}+\varepsilon_{3}\right)} & E_{ \pm\left(\varepsilon_{2}+\varepsilon_{3}\right)}+E_{ \pm\left(\varepsilon_{1}-\varepsilon_{4}\right)}+E_{ \pm\left(\varepsilon_{1}+\varepsilon_{4}\right)}
\end{array}
$$

where the \pm signs in the right column are correlated on each line. To realize the full maximal bosonic subalgebra $S L(2, \mathbf{R}) \oplus G_{2}$ of $G(3 ; 0)$ as a subalgebra of $S O(8) \oplus S p(4)$, we need to realize also $S L(2, \mathbf{R})$ as a subalgebra of $S p(4, \mathbf{R})$. This problem was already encountered when dealing with the case of $F(4)$. There are 3 possible inequivalent embeddings, corresponding to the decompositions of the $\mathbf{5}$ of $S O(2,3)$, given in (C.9), with associated generators given in (C.10). In total, the maximal bosonic subalgebra $S L(2) \times G_{2}$ of $G(3 ; 0)$ can be embedded in three inequivalent ways in the maximal bosonic subalgebra of $O S p(8 \mid 4, \mathbf{R})$.

2. The fermionic generators of $G(3 ; 0)$ transform under the representation $(2,7)$ of $S L(2, \mathbf{R}) \times G_{2}$. We shall now investigate whether fermionic generators can be realized in terms of linear combinations of the fermionic generators of $O S p(8 \mid 4, \mathbf{R})$, for each of the three embeddings of (C.9), and (C.10).

(a) Under $\operatorname{Sp}(2, \mathbf{R}) \oplus G_{2}$, the fermionic generators should transform as a $(\mathbf{2}, \mathbf{7})$, leaving the following unique realization,

$$
\begin{array}{ll}
E_{ \pm \varepsilon_{i} \pm \delta_{1}} & i=1,2,3 \\
E_{ \pm\left(\varepsilon_{4}+\delta_{1}\right)}+E_{ \pm\left(-\varepsilon_{4}+\delta_{1}\right)} &
\end{array}
$$

The \pm signs on the second line are correlated, while those on the first line are not. The generators $E_{ \pm\left(\varepsilon_{4}+\delta_{1}\right)}-E_{ \pm\left(-\varepsilon_{4}+\delta_{1}\right)}$ are singlets under $G_{2}$, while $E_{ \pm \varepsilon_{i} \pm \delta_{2}}$ with $i=1,2,3,4$ are singlets under $S p(2, \mathbf{R})$. The 14 generators of (C.14) have indeed the correct transformation properties under $S L(2, \mathbf{R}) \times G_{2}$ and constitute the only viable candidates for the representation of the fermionic generators of $G(3 ; 0)$ inside $O S p(8 \mid 4, \mathbf{R})$.

(b) In this case, the representation of $S p(2, \mathbf{R})$ is a sum of two doublets under $S p(2, \mathbf{R})$. This leaves the following possible realizations of the positive root fermionic generators,

$$
\begin{aligned}
& a E_{ \pm \varepsilon_{i}+\delta_{1}}+b E_{ \pm \varepsilon_{i}+\delta_{2}}, \quad i=1,2,3 \\
& a\left(E_{\varepsilon_{4}+\delta_{1}}+E_{-\varepsilon_{4}+\delta_{1}}\right)+b\left(E_{\varepsilon_{4}+\delta_{2}}+E_{-\varepsilon_{4}+\delta_{2}}\right)
\end{aligned}
$$

Here, $a$ and $b$ are two constants which we may choose to satisfy $|a|^{2}+|b|^{2}=2$. The fact that the same constants occur in the seven linear combinations above is required 
by $G_{2}$ covariance. It is manifest that these generators also form 7 doublets under the action of the $S p(2, \mathbf{R})$ root generators $E_{ \pm 2 \delta_{1}}+E_{ \pm 2 \delta_{2}}$, for any values of $a, b$.

(c) The representation of $\operatorname{Sp}(2, \mathbf{R})$ is irreducible and of dimension 4, and cannot be used to realize the doublet needed in the representation $(\mathbf{2}, \mathbf{7})$. Hence the fermionic generators of $G(3 ; 0)$ cannot be realized in this case.

3. Having now determined the structure of the possible embeddings of the maximal bosonic subalgebras and the fermionic generators, it remains to analyze the structure of the anti-commutators of these generators, and see whether they close onto the bosonic generators of $G(3 ; 0)$; we shall show that they do not.

(a) The anticommutators $\left\{E_{ \pm \varepsilon_{i}+\delta_{1}}, E_{ \pm \varepsilon_{j}-\delta_{1}}\right\} \sim E_{ \pm \varepsilon_{i} \pm \varepsilon_{j}}$ for all $i \neq j=1,2,3$ produce the roots of the full orthogonal algebra $S O(6)$, which is, however, not a subalgebra of $G_{2}$. (The anti-commutators $\left\{E_{ \pm \varepsilon_{i}+\delta_{1}}, E_{\varepsilon_{4}-\delta_{1}}+E_{-\varepsilon_{4}-\delta_{1}}\right\} \sim E_{ \pm \varepsilon_{i}+\varepsilon_{4}}+E_{ \pm \varepsilon_{i}-\varepsilon_{4}}$ further enlarges this algebra.) As a result, the fermion generators do not properly close to form $G(3)$.

(b) The following anti-commutators

$$
\left\{a E_{ \pm \varepsilon_{i}+\delta_{1}}+b E_{ \pm \varepsilon_{i}+\delta_{2}}, a E_{ \pm \varepsilon_{j}-\delta_{1}}+b E_{ \pm \varepsilon_{j}-\delta_{2}}\right\} \sim E_{ \pm \varepsilon_{i} \pm \varepsilon_{j}} \quad i \neq j
$$

again produces all of $S O(6) \not \subset G_{2}$. Similarly, the anti-commutators of the fermionic generators do also not properly close onto $S p(2, \mathbf{R})$. Thus, the fermion generators do not properly close to form $G(3 ; 0)$.

This concludes the proof that $G(3 ; 0)$ cannot be a subalgebra of $O S p(8 \mid 4, \mathbf{R})$.

\section{C.4.4 $D(2,1 ; c)$ a subalgebra of $O S p(8 \mid 4)$ implies $c=1,-2$, or $-1 / 2$}

The maximal bosonic subalgebra of the real form $D(2,1 ; c ; p)$ of $D(2,1 ; c)$ for $p=0,1,2$ is given by $S L(2, \mathbf{R}) \oplus S O(p, 4-p)$.

1. We have the following results for the embeddings into the maximal bosonic subalgebras of $O S p\left(8^{*} \mid 4\right)$ and $O S p(8 \mid 4, \mathbf{R})$,

$$
\begin{array}{rll}
S L(2, \mathbf{R}) \oplus S O(4) & \subset & S O(2,6) \oplus S p(4) \\
S L(2, \mathbf{R}) \oplus S O(4) & \subset & S O(8) \oplus S p(4, \mathbf{R}) \\
S L(2, \mathbf{R}) \oplus S O(1,3) & \subset & S O(2,6) \oplus S p(4) \\
S L(2, \mathbf{R}) \oplus S O(1,3) & \not \subset & S O(8) \oplus S p(4, \mathbf{R}) \\
S L(2, \mathbf{R}) \oplus S O(2,2) & \not \subset & S O(2,6) \oplus S p(4) \\
S L(2, \mathbf{R}) \oplus S O(2,2) & \not \subset & S O(8) \oplus S p(4, \mathbf{R})
\end{array}
$$


2. Next, we show that two of the remaining possible embeddings

$$
\begin{aligned}
S L(2, \mathbf{R}) \oplus S O(4) & \subset S O(2,6) \\
S L(2, \mathbf{R}) \oplus S O(1,3) & \subset S O(2,6)
\end{aligned}
$$

are ruled out for the following reason. In either case, the bosonic subalgebra $\mathcal{S}$ must be realized in $S O(2,6)$ in such a way that the fermionic generators transform under $\mathcal{S}$ in the representation $(\mathbf{2}, \mathbf{2}, \mathbf{2})$. The representation of the fermionic generators would have to involve an entire 8 multiplet of $S O(2,6)$, with generators $E_{ \pm \varepsilon_{i}+\delta}$ for $i=1,2,3,4$ for some $\delta$. Since the desired subalgebra $D(2,1 ; c)$ is to be simple, the opposite roots must be contained in the subalgebra as well, so we would also need to include the fermion contents $E_{ \pm \varepsilon_{i}-\delta}$ for $i=1,2,3,4$, giving a total of 16 fermionic generators, which does not fit with $D(2,1 ; c)$.

Next, we show that two more of the remaining possible embeddings

$$
\begin{aligned}
S O(3) \oplus S O(3) & \subset S p(4) \\
S L(2, \mathbf{R}) \oplus S O(3) & \subset S p(4, \mathbf{R})
\end{aligned}
$$

are also ruled out. In both cases, the fermion contents must include a full $\mathbf{4}$ of $S p(4)$ or $S p(4, \mathbf{R})$, given by the generators $E_{ \pm \varepsilon \pm \delta_{a}}$ with $a=1,2$. Under anti-commutation, however, these fermionic generators will reproduce the entire $S p(4)$, or $S p(4, \mathbf{R})$, and not just the desired bosonic subalgebras.

Assembling the results from the above arguments, we see that the case on the third line of (C.17) is ruled out altogether, so that the only real form that remains to be considered is $D(2,1 ; c ; 0)$, with the following possible embeddings of the maximal bosonic subalgebras,

$$
\begin{array}{cl}
O S p(8 \mid 4, \mathbf{R}) \quad\left\{\begin{array}{c}
S O(3) \oplus S O(3) \subset S O(8) \\
S L(2, \mathbf{R}) \subset S p(4, \mathbf{R})
\end{array}\right. \\
O S p\left(8^{*} \mid 4\right) & \left\{\begin{array}{c}
S L(2, \mathbf{R}) \oplus S O(3) \subset S O(2,6) \\
S O(3) \subset S p(4)
\end{array}\right.
\end{array}
$$

The two cases of (C.20) may be viewed as different real forms of the same embedding structure over $\mathbf{C}$. We shall discuss the first case in detail; the second case is completely analogous.

For the first embedding case in (C.20), there are two further different possible embeddings of $S O(3) \oplus S O(3)$ into $S O(8)$, which may be distinguished by the branching rules of the fundamental representation 8 of $S O(8)$,

$$
\begin{aligned}
& \text { (1) } \quad \mathbf{8}=\mathbf{4} \oplus \mathbf{1} \oplus \mathbf{1} \oplus \mathbf{1} \oplus \mathbf{1} \\
& \text { (2) } \quad \mathbf{8}=\mathbf{4} \oplus \mathbf{4}
\end{aligned}
$$


Similarly, there are three possible embeddings of $S L(2, \mathbf{R})=S p(2, \mathbf{R})$ into $S p(4, \mathbf{R})$, which already listed for the case of $F(4)$ in (C.9). It is immediate, however, that case (c) is ruled out, as we seek a fermionic representation transforming under the $\mathbf{2}$ of this $S p(2, \mathbf{R})$, not an irreducible 4. Combining all, we have 4 remaining possible cases, which we can label (1a), (1b), (2a), (2b), according to the labels for embedding into $S O(8)$ and $S p(4, \mathbf{R})$.

3. We shall now work out these cases one by one.

- In case (a1), the generators produce the superalgebra $O S p(4 \mid 2, \mathbf{R})=D(2,1 ; 1 ; 0)$.

- In case (1b), the bosonic generators are $E_{ \pm \varepsilon_{1} \pm \varepsilon_{2}}$ for the $S O(4)$ part, and $E_{ \pm 2 \delta_{1}}+E_{ \pm 2 \delta_{2}}$ for the $S L(2, \mathbf{R})$ part. The fermionic generators must then be,

$$
\begin{array}{ll}
a E_{ \pm \varepsilon_{i}+\delta_{1}}+b E_{ \pm \varepsilon_{i}+\delta_{2}} & i=1,2 \\
a E_{ \pm \varepsilon_{i}-\delta_{1}}+b E_{ \pm \varepsilon_{i}-\delta_{2}} & i=1,2
\end{array}
$$

where $a, b$ are two constants. The anti-commutators of the generators with opposite $\delta_{a}$ correctly reproduce the bosonic generators of $S O(4)$. But the anti-commutators

$$
\left\{a E_{\varepsilon+\delta_{1}}+b E_{\varepsilon+\delta_{2}}, a E_{-\varepsilon+\delta_{1}}+b E_{-\varepsilon+\delta_{2}}\right\}=a^{2} E_{2 \delta_{1}}+b^{2} E_{2 \delta_{2}}+2 a b E_{\delta_{1}+\delta_{2}}
$$

do not correctly reproduce the generators of $S L(2, \mathbf{R})$ for any choice of $a, b$. The absence of the generators $E_{\delta_{1}+\delta_{2}}$ would require $a b=0$, while reproducing $E_{ \pm 2 \delta_{1}}+E_{ \pm 2 \delta_{2}}$ would require $a=b$, implying $a=b=0$, and thus no fermionic generators. This rules out case (1b).

- In case (2a), the argument is similar to the one given in case (1b), but the roles of the bosonic subalgebras $S O(4)$ and $S L(2, \mathbf{R})$ are interchanged. The bosonic generators are now $E_{ \pm 2 \delta_{1}}$ for the $S L\left(2, \mathbf{R}\right.$ ) part, and $E_{ \pm \varepsilon_{1} \pm \varepsilon_{2}}+E_{ \pm \varepsilon_{3} \pm \varepsilon_{4}}$ (with correlated \pm signs between the two terms). The fermionic generators must form a doublet under $S L(2, \mathbf{R})$, and an irreducible 4 under $S O(4)$, which fixes them to be

$$
\begin{array}{ll}
a E_{ \pm \varepsilon_{i}+\delta_{1}}+b E_{ \pm \varepsilon_{2+i}+\delta_{1}} & i=1,2 \\
a E_{ \pm \varepsilon_{i}-\delta_{1}}+b E_{ \pm \varepsilon_{2+i}-\delta_{1}} & i=1,2
\end{array}
$$

where $a, b$ are two constants. The anti-commutators for opposite $\varepsilon$ correctly reproduce the generators $E_{ \pm 2 \delta_{1}}$, but the commutators for opposite $\delta$ give, for example,

$$
\begin{aligned}
& \left\{a E_{\varepsilon_{1}+\delta_{1}}+b E_{\varepsilon_{3}+\delta_{1}}, a E_{\varepsilon_{2}-\delta_{1}}+b E_{\varepsilon_{4}-\delta_{1}}\right\} \\
& \quad a^{2} E_{\varepsilon_{1}+\varepsilon_{2}}+b^{2} E_{\varepsilon_{3}+\varepsilon_{4}}+a b\left(E_{\varepsilon_{1}+\varepsilon_{4}}-E_{\varepsilon_{2}+\varepsilon_{3}}\right)
\end{aligned}
$$

Absence of the generators $E_{\varepsilon_{1}+\varepsilon_{4}}$ and $E_{\varepsilon_{2}+\varepsilon_{3}}$ requires $a b=0$, while recovering the correct generators of the type $E_{ \pm \varepsilon_{1} \pm \varepsilon_{2}}+E_{ \pm \varepsilon_{3} \pm \varepsilon_{4}}$ requires $a^{2}=b^{2}$, which implies $a=b=0$, and hence no fermionic generators. This rules out case (2a). 
- Finally, in case $(2 \mathrm{~b})$, the bosonic generators of the $S O(4)$ part are $E_{ \pm \varepsilon_{1} \pm \varepsilon_{2}}+E_{ \pm \varepsilon_{3} \pm \varepsilon_{4}}$, while those of the $S L(2, \mathbf{R})$ part are $E_{ \pm 2 \delta_{1}}+E_{ \pm 2 \delta_{2}}$. The fermionic generators must be,

$$
\begin{array}{ll}
X_{ \pm i}^{+} \equiv a E_{ \pm \varepsilon_{i}+\delta_{1}}+b E_{ \pm \varepsilon_{i}+\delta_{2}}+c E_{ \pm \varepsilon_{i}^{\prime}+\delta_{1}}+d E_{ \pm \varepsilon_{i}^{\prime}+\delta_{2}} & i=1,2 \\
X_{ \pm i}^{-} \equiv a E_{ \pm \varepsilon_{i}-\delta_{1}}+b E_{ \pm \varepsilon_{i}-\delta_{2}}+c E_{ \pm \varepsilon_{i}^{\prime}-\delta_{1}}+d E_{ \pm \varepsilon_{i}^{\prime}-\delta_{2}} & i=1,2
\end{array}
$$

where $\varepsilon_{1}^{\prime}=\varepsilon_{3}$ and $\varepsilon_{2}^{\prime}=\varepsilon_{4}$. Here, $a, b, c, d$ are constants which are independent of $i$ and of \pm by $S O(4)$ invariance and must be the same on the two lines in view of $S L(2, \mathbf{R})$-invariance. The anti-commutators for opposite $\delta$, and opposite $\varepsilon$ give, for example,

$$
\begin{aligned}
\left\{X_{+1}^{+}, X_{+2}^{-}\right\} & =\left(a^{2}+b^{2}\right) E_{\varepsilon_{1}+\varepsilon_{2}}+\left(c^{2}+d^{2}\right) E_{\varepsilon_{3}+\varepsilon_{4}}+a c E_{\varepsilon_{1}+\varepsilon_{3}}+b d E_{\varepsilon_{2}+\varepsilon_{4}} \\
\left\{X_{+1}^{+}, X_{-1}^{+}\right\} & =\left(a^{2}+c^{2}\right) E_{2 \delta_{1}}+\left(b^{2}+d^{2}\right) E_{2 \delta_{2}}+(a b+c d) E_{\delta_{1}+\delta_{2}}
\end{aligned}
$$

Absence of the undesirable generators $E_{\varepsilon_{1}+\varepsilon_{3}}$, and $E_{\varepsilon_{2}+\varepsilon_{4}}$ on the first line requires that $a c=b d=0$. Absence of the undesirable generators $E_{\delta_{1}+\delta_{2}}$ on the second line requires $a b+c d=0$. Fully recovering the closure onto the bosonic subalgebra requires the conditions,

$$
\begin{aligned}
& 0=a^{2}+b^{2}-c^{2}-d^{2}=a^{2}-b^{2}+c^{2}-d^{2} \\
& 0=a b+c d=a c=b d
\end{aligned}
$$

The first line is equivalent to $a^{2}=d^{2}, b^{2}=c^{2}$. If $a \neq 0$, we have $c=b=0$ by (C.28). Choosing $a=d=1$, without loss of generality, the fermionic generators reduce to

$$
\begin{array}{rlrl}
X_{ \pm i}^{+} & \equiv E_{ \pm \varepsilon_{i}+\delta_{1}}+E_{ \pm \varepsilon_{i}^{\prime}+\delta_{2}} & i=1,2 \\
X_{ \pm i}^{-} \equiv E_{ \pm \varepsilon_{i}-\delta_{1}}+E_{ \pm \varepsilon_{i}^{\prime}-\delta_{2}} & i=1,2
\end{array}
$$

This gives the structure relations of $O S p(4 \mid 2, \mathbf{R})$, embedded into $O S p(8 \mid 4, \mathbf{R})$ as the diagonal subalgebra of $O S p(4 \mid 2, \mathbf{R}) \oplus O S p(4 \mid 2, \mathbf{R})$, where the first term is based on the root generators $E_{ \pm \varepsilon_{1} \pm \delta_{1}}+E_{ \pm \varepsilon_{3} \pm \delta_{2}}$ and the second on the root generators $E_{ \pm \varepsilon_{2} \pm \delta_{1}}+E_{ \pm \varepsilon_{4} \pm \delta_{2}}$. If $b \neq 0$, we obtain an equivalent realization to the case $a^{2}=d^{2} \neq 0$, again giving $O S p(4 \mid 2, \mathbf{R})$, embedded into $\operatorname{OSp}(8 \mid 4, \mathbf{R})$ as the diagonal subalgebra of $O S p(4 \mid 2, \mathbf{R}) \oplus O S p(4 \mid 2, \mathbf{R})$.

In conclusion, cases (1b) and (2a) are ruled out, while for cases (1a), and (2b) the subalgebra is $D(2,1 ; 1 ; 0)=O S p(4 \mid 2, \mathbf{R})$. In case (1a), there is room for also a second copy, and the corresponding embedding is actually via two commuting superalgebras,

$$
O S p(4 \mid 2, \mathbf{R}) \oplus O S p(4 \mid 2, \mathbf{R}) \subset O S p(8 \mid 4, \mathbf{R})
$$

The proof for the second embedding case in (C.20) is completely analogous, and offers an outcome analogous to that of (C.30). The result is that $D(2,1 ; c ; 0)$ can be embedded into 
$O S p\left(8^{*} \mid 4\right)$ only when $c=-2,-1 / 2$, in which case we have $D(2,1 ; c ; 0)=O S p\left(4^{*} \mid 2\right)$ for $c=-2,-1 / 2$. The embedding of $O S p\left(4^{*} \mid 2\right)$ can be via a single block, or via two commuting superalgebras,

$$
O S p\left(4^{*} \mid 2\right) \oplus O S p\left(4^{*} \mid 2\right) \subset O S p\left(8^{*} \mid 4\right)
$$

The exceptional basic Lie superalgebra $D(2,1 ; c)$, or any real form $D(2,1 ; c ; p)$ thereof, is never a subalgebra of $O S p(8 \mid 4, \mathbf{R})$ when $c \neq 1$, and is never a subalgebra of $O S p\left(8^{*} \mid 4\right)$ when $c \neq-2,-1 / 2$. 


\section{Superalgebra structure of the M-theory solutions}

The infinitesimal symmetry generators of a particular half-BPS solution form a superalgebra with bosonic and fermionic generators. On a supergravity solution we identify the bosonic generators with isometries of the metric and the fermionic generators with unbroken supersymmetries. The BPS equations for M-theory are given by,

$$
\nabla_{M} \varepsilon+\frac{1}{288}\left(\Gamma_{M}^{N P Q R}-8 \delta_{M}^{N} \Gamma^{P Q R}\right) F_{N P Q R} \varepsilon=0
$$

We generally follow the conventions and notations of [10]. Here, $\varepsilon$ is an 11-dimensional Majorana spinor, and $\nabla_{M}$ is the covariant derivative with respect to the Levi-Civita connection for $g_{M N}$. Given any $\varepsilon$ and $\varepsilon^{\prime}$ satisfying (D.1), it follows from the BPS equations that the vector $v_{M}$, defined below, satisfies the Killing equation,

$$
\begin{aligned}
v_{M} & \equiv e_{M}^{A}\left(\bar{\varepsilon} \Gamma_{A} \varepsilon^{\prime}\right) \\
0 & =\nabla_{M} v_{N}+\nabla_{N} v_{M}
\end{aligned}
$$

This result is established by computing the following expression using the BPS equations,

$$
3 \nabla_{M} v_{N}=\left(\bar{\varepsilon} \Gamma^{Q R} \varepsilon^{\prime}\right) F_{M N Q R}
$$

and symmetrizing in $M, N$. The result may be interpreted as a composition law for supersymmetry transformations $\varepsilon$ and $\varepsilon^{\prime}$, which closes onto Killing vector $v_{M}$, and may be used to give a constructive derivation of the superalgebra [21].

We apply the above methods to prove that the invariance superalgebra of the M-theory half-BPS solutions of $[10]$ is $D(2,1 ; c) \times D(2,1 ; c)$. Here, $D(2,1 ; c)$ is the 1 -parameter family of exceptional basic classical Lie superalgebras, and the real number $c$ will be related to certain parameters of the solutions. We refer to [10] for additional details on this solution.

\section{D.1 Calculation of the Killing vectors}

The Ansatz for $S O(2,2) \times S O(4)_{2} \times S O(4)_{3}$-invariant solutions to M-theory on a spacetime $A d S_{3} \times S_{2}^{3} \times S_{3}^{3}$ warped over a two-dimensional Riemann surface $\Sigma$ with boundary is given as follows. The 11-dimensional metric $d s^{2}$ associated with the Minkowski metric $\eta_{A B}=(-+\cdots+)$ on the orthonormal frame $e^{A}$, with $A, B=0,1,2, \cdots, 9, \downarrow=10$, is given by $d s^{2}=\eta_{A B} e^{A} \otimes e^{B}$. 
The components of the orthonormal frame $e^{A}=d x^{M} e_{M}^{A}$ for this Ansatz take the form,

$$
\begin{array}{lllc}
e^{a_{1}}=f_{1} \hat{e}^{a_{1}} & a_{1}=0,1,2 & A d S_{3} \\
e^{a_{2}}=f_{2} \hat{e}^{a_{2}} & a_{2}=3,4,5 & S_{2}^{3} \\
e^{a_{3}}=f_{1} \hat{e}^{a_{3}} & a_{3}=6,7,8 & S_{3}^{3} \\
e^{a} & & a=9, \downarrow=10 & \Sigma
\end{array}
$$

An explicit expression for the invariant 4-form field strength $F$ may be found in [10] but will not be needed here. A choice of well-adapted Dirac matrices is given by

$$
\begin{array}{ll}
\Gamma^{a_{1}}=\gamma^{a_{1}} \otimes I_{2} \otimes I_{2} \otimes \sigma^{1} \otimes \sigma^{3} & \sigma^{2}=-i \gamma^{0}=\gamma^{3}=\gamma^{6}=\gamma^{\natural} \\
\Gamma^{a_{2}}=I_{2} \otimes \gamma^{a_{2}} \otimes I_{2} \otimes \sigma^{2} \otimes \sigma^{3} & \sigma^{1}=\gamma^{1}=\gamma^{4}=\gamma^{7}=\gamma^{9} \\
\Gamma^{a_{3}}=I_{2} \otimes I_{2} \otimes \gamma^{a_{3}} \otimes \sigma^{3} \otimes \sigma^{3} & \\
\Gamma^{a}=I_{2} \otimes I_{2} \otimes I_{2} \otimes I_{2} \otimes \gamma^{a} &
\end{array}
$$

where $a_{1}=0,1,2, a_{2}=3,4,5, a_{3}=6,7,8$, and $a=9, \sharp$. The supersymmetry parameter $\varepsilon$ may be decomposed on a basis of $S O(2,2) \times S O(4)_{2} \times S O(4)_{3}$-invariant Killing spinors $\chi^{\eta_{1}, \eta_{2}, \eta_{3}}$, with $\eta_{1}, \eta_{2}, \eta_{3}= \pm$

$$
\varepsilon=\chi^{\eta_{1}, \eta_{2}, \eta_{3}} \zeta_{\eta_{1}, \eta_{2}, \eta_{3}}
$$

In total, there is a 16-dimensional space of solutions $\varepsilon$, which may be parametrized by picking definite values for $\eta_{1}, \eta_{2}, \eta_{3}= \pm$, and using a two-fold degeneracy of $\zeta$. It suffices to solve the BPS equations for the above Ansatz only in part [10] to obtain the metric factors $f_{1}, f_{2}, f_{3}$ in terms of $\zeta$. They are given by,

$$
\begin{aligned}
& 2 c_{1} f_{1}=\zeta^{\dagger}\left(I_{2} \otimes I_{2}\right) \zeta \\
& 2 c_{2} f_{2}=-\zeta^{\dagger}\left(\sigma^{3} \otimes I_{2}\right) \zeta \\
& 2 c_{3} f_{3}=\zeta^{\dagger}\left(\sigma^{2} \otimes I_{2}\right) \zeta
\end{aligned}
$$

Here, $c_{1}, c_{2}, c_{3}$ are real integration constants, which are related by $c_{1}+c_{2}+c_{3}=0$. (In fact, we shall show that the constant $c$ in the invariance super algebra $D(2,1 ; c) \times D(2,1 ; c)$ is given by $c=c_{2} / c_{1}$, or equivalently, by any other ratio of two $c_{i}$.)

The 8 linearly independent Killing spinors $\chi^{\eta_{1}, \eta_{2}, \eta_{3}}$ may be normalized as follows,

$$
\begin{array}{rlrl}
\chi^{\eta_{1}, \eta_{2}, \eta_{3}} & =\chi_{1}^{\eta_{1}} \otimes \chi_{2}^{\eta_{2}} \otimes \chi_{3}^{\eta_{3}} & \\
\bar{\chi}_{1}^{\eta_{1}} \chi_{1}^{\eta_{1}^{\prime}} & =i \delta_{\eta_{1}, \eta_{1}^{\prime}} & i=2,3 \\
\left(\chi_{i}^{\eta_{i}}\right)^{\dagger} \chi_{i}^{\eta_{i}^{\prime}} & =\delta_{\eta_{i}, \eta_{i}^{\prime}}
\end{array}
$$


where we define, as usual for Minkowski signature spinors, $\bar{\chi}_{1}^{\eta}=\left(\chi_{1}^{\eta}\right)^{\dagger} \gamma^{0}$. The Killing vectors of the M-theory solutions may now be evaluated by considering the combinations $\bar{\varepsilon}^{\prime} \Gamma^{A} \varepsilon$ (with Lorentz frame indices). It will be convenient to decompose these according to the product $A d S_{3} \times S_{2}^{3} \times S_{3}^{3} \times \Sigma$. One finds,

$$
\begin{aligned}
& \bar{\varepsilon} \Gamma^{a_{1}} \varepsilon^{\prime}=\left(\bar{\chi}_{1}^{\eta_{1}} \gamma^{a_{1}} \chi_{1}^{\eta_{1}^{\prime}}\right) \delta_{\eta_{2}, \eta_{2}^{\prime}} \delta_{\eta_{3}, \eta_{3}^{\prime}}\left(\zeta^{\dagger} I_{2} \otimes I_{2} \zeta\right) \\
& \bar{\varepsilon} \Gamma^{a_{2}} \varepsilon^{\prime}=\delta_{\eta_{1}, \eta_{1}^{\prime}}\left(\left(\chi_{2}^{\eta_{2}}\right)^{\dagger} \gamma^{a_{2}} \chi_{2}^{\eta_{2}^{\prime}}\right) \delta_{\eta_{3}, \eta_{3}^{\prime}}\left(\zeta^{\dagger}\left(i \sigma^{3}\right) \otimes I_{2} \zeta\right) \\
& \bar{\varepsilon} \Gamma^{a_{3}} \varepsilon^{\prime}=\delta_{\eta_{1}, \eta_{1}^{\prime}} \delta_{\eta_{2}, \eta_{2}^{\prime}}\left(\left(\chi_{3}^{\eta_{3}}\right)^{\dagger} \gamma^{a_{3}} \chi_{3}^{\eta_{3}^{\prime}}\right)\left(\zeta^{\dagger}\left(-i \sigma^{2}\right) \otimes I_{2} \zeta\right) \\
& \bar{\varepsilon} \Gamma^{a} \varepsilon^{\prime}=\delta_{\eta_{1}, \eta_{1}^{\prime}} \delta_{\eta_{2}, \eta_{2}^{\prime}} \delta_{\eta_{3}, \eta_{3}^{\prime}}\left(\zeta^{\dagger} \sigma^{1} \otimes \sigma^{3} \sigma^{a} \zeta\right)
\end{aligned}
$$

Using the expressions for the metric factors $f_{i}$ of (D.7), we may convert the bilinears in $\zeta$ in the above expressions in terms of metric factors $f_{i}$ and the constants $c_{i}$. Also, using the fact that $\zeta^{\dagger} \sigma^{1} \otimes \sigma^{3} \sigma^{a} \zeta=0$ for $a=9$, , we obtain,

$$
\begin{aligned}
\bar{\varepsilon} \Gamma^{a_{1}} \varepsilon^{\prime} & =2 c_{1} f_{1}\left(\bar{\chi}_{1} \gamma^{a_{1}} \chi_{1}^{\prime}\right) \delta_{\eta_{2}, \eta_{2}^{\prime}} \delta_{\eta_{3}, \eta_{3}^{\prime}} \\
\bar{\varepsilon} \Gamma^{a_{2}} \varepsilon^{\prime} & =2 c_{2} f_{2} \delta_{\eta_{1}, \eta_{1}^{\prime}}\left(-i \chi_{2}^{\dagger} \gamma^{a_{2}} \chi_{2}^{\prime}\right) \delta_{\eta_{3}, \eta_{3}^{\prime}} \\
\bar{\varepsilon} \Gamma^{a_{3}} \varepsilon^{\prime} & =2 c_{3} f_{3} \delta_{\eta_{1}, \eta_{1}^{\prime}} \delta_{\eta_{2}, \eta_{2}^{\prime}}\left(-i \chi_{3}^{\dagger} \gamma^{a_{3}} \chi_{3}^{\prime}\right) \\
\bar{\varepsilon} \Gamma^{a} \varepsilon^{\prime} & =0
\end{aligned}
$$

It remains to exhibit the relations between the bilinears in $\chi_{i}$ and the Killing vectors on the unit radius spaces $A d S_{3}, S_{2}^{3}$, and $S_{3}^{3}$.

\section{D.2 Normalization of the unit radius $A d S_{3}$ Killing vectors}

For $A d S_{3}$, the Killing spinor equations were derived in [10], and may be expressed as follows in terms of 4-component Dirac spinors $\chi_{1}$,

$$
\left(d+\omega_{1}^{(t)}\right) \chi_{1}=\left(d+\frac{1}{4}\left(\omega_{1}\right)_{a_{1} b_{1}} \gamma^{a_{1} b_{1}} \otimes I_{2}+\frac{1}{2}\left(e_{1}\right)_{a_{1}} \gamma^{a_{1}} \otimes \sigma^{3}\right) \chi_{1}=0
$$

where $a_{1}, b_{1}=0,1,2$, and $\omega_{1}^{(t)}=U_{1}^{-1} d U_{1}$ is parametrized by $U_{1}$ in the spinor representation of $S O(2,2)$. The Killing spinor equations for 2-component Weyl spinors $\chi^{\eta}$ [10], may be obtained from the equations for $\chi_{1}$ by projection onto definite chiralities. The general solution for Dirac spinors $\chi_{1}$, and for the Weyl spinors $\chi_{1}^{\eta}$ are given by

$$
\begin{aligned}
& \chi_{1}=U_{1} \varepsilon_{1} \\
& \chi_{1}^{\eta} \equiv P_{\eta} U_{1} \varepsilon_{1} \\
& P_{\eta} \equiv \frac{1}{2}\left(1+\eta I_{2} \otimes \sigma^{3}\right)
\end{aligned}
$$


Here, $\varepsilon_{1}$ is an arbitrary constant 4-component spinor, which we conveniently normalize to $\bar{\varepsilon}_{1} \varepsilon_{1}=1$, so that the normalization condition (D.8) for $\chi_{1}$ holds. Since $P_{\eta}$ commutes with the $S O(2,2)$ generators in the spinor representation, the actions of the simple factors $S O(2,1)_{+} \times$ $S O(2,1)_{-}=S O(2,2)$ may be identified with the generators $P_{ \pm} \gamma^{a_{1} b_{1}}$. The Killing spinors $\chi_{1}^{+}$ and $\chi_{1}^{-}$transform under $S O(2,1)_{+} \otimes S O(2,1)_{-}$respectively as $(\mathbf{2}, \mathbf{1})$ and $(\mathbf{1}, \mathbf{2})$. It is now immediate to evaluate the Killing vector combinations $\bar{\chi}_{1} \gamma^{a_{1}} \chi_{1}^{\prime}$, and we have

$$
\begin{aligned}
\bar{\chi}_{1}^{\eta} \gamma^{a_{1}} \chi_{1}^{-\eta} & =0 \\
\left(v_{1}\right)_{ \pm}^{a_{1}} \equiv \bar{\chi}_{1}^{ \pm} \gamma^{a_{1}} \chi_{1}^{ \pm} & =\varepsilon_{1}^{\dagger} U_{1}^{\dagger} \gamma^{0} \gamma^{a_{1}} U_{1} P_{ \pm} \varepsilon_{1}
\end{aligned}
$$

The $\left(v_{1}\right)_{+}^{a_{1}}$ and $\left(v_{1}\right)_{-}^{a_{1}}$ are respectively the Killing vectors generating the isometry groups $S O(2,1)_{+}$and $S O(2,1)_{-}$on $A d S_{3}$ of unit radius.

\section{D.3 Normalization of the unit radius $S^{3}$ Killing vectors}

Similarly, for $S_{i}^{3}$, with $i=2,3$, the Killing spinor equations, expressed in terms of 4 component Dirac spinors, are given by,

$$
\left(d+\omega_{i}^{(t)}\right) \chi_{i}\left(d+\frac{1}{4}\left(\omega_{i}\right)_{a_{i} b_{i}} \gamma^{a_{i} b_{i}} \otimes I_{2}+\frac{i}{2}\left(e_{i}\right)_{a_{i}} \gamma^{a_{i}} \otimes \sigma^{3}\right) \chi_{i}=0
$$

where $a_{2}, b_{2}=3,4,5, a_{3}, b_{3}=6,7,8$, and $\omega_{i}^{(t)}=U_{i}^{\dagger} d U_{i}$ is parametrized by $U_{i}$ in the spinor representation of $S O(4)_{i}$. The general solution $\chi_{i}$ and the chiral solutions $\chi_{i}^{\eta}$ are given by

$$
\chi_{i}=U_{i} \varepsilon_{i} \quad \chi_{i}^{\eta} \equiv P_{\eta} U_{i} \varepsilon_{i} \quad P_{\eta} \equiv \frac{1}{2}\left(1+\eta I_{2} \otimes \sigma^{3}\right)
$$

Here, $\varepsilon_{i}$ are arbitrary constant spinors, which we conveniently normalize to $\varepsilon_{i}^{\dagger} \varepsilon_{i}=1$, so that the normalization conditions (D.8) for $\chi_{i}$ hold. Since $P_{\eta}$ commutes with the $S O(4)_{i}$ generators in the spinor representation, the actions of the simple factors $S U(2)_{i+} \times S U(2)_{i-}=$ $S O(4)_{i}$ may be identified with the rotations $P_{ \pm} \gamma^{a_{i} b_{i}}$. The Killing spinors $\chi_{i}^{+}$and $\chi_{i}^{-}$transform under $S U(2)_{i+} \otimes S U(2)_{i-}$ respectively as $(\mathbf{2}, \mathbf{1})_{i}$ and $(\mathbf{1}, \mathbf{2})_{i}$. It is now immediate to evaluate the Killing vector combinations $\chi_{i}^{\dagger} \gamma^{a_{i}} \chi_{i}^{\prime}$, and we have

$$
\begin{aligned}
\left(\chi_{i}^{\eta}\right)^{\dagger} \gamma^{a_{i}} \chi_{i}^{\eta} & =0 & \eta= \pm \\
\left(v_{i}\right)_{ \pm}^{a_{i}} \equiv\left(\chi_{i}^{\mp}\right)^{\dagger} \gamma^{a_{i}} \chi_{i}^{ \pm} & =\varepsilon_{i}^{\dagger} U_{i}^{\dagger} \gamma^{a_{i}} U_{i} P_{ \pm} \varepsilon_{i} &
\end{aligned}
$$

The $\left(v_{i}\right)_{+}^{a_{i}}$ and $\left(v_{i}\right)_{-}^{a_{i}}$ are respectively the Killing vectors generating the isometry groups $S U(2)_{i+}$ and $S U(2)_{i-}$ on the spheres of unit radii $S_{i}^{3}$. 


\section{D.4 Summary of results}

Combining all results, we find that the spinor bilinears evaluate as follows,

$$
\begin{aligned}
\bar{\varepsilon} \Gamma^{a_{1}} \varepsilon^{\prime} & =2 c_{1} f_{1}\left(v_{1}\right)_{ \pm}^{a_{1}} \delta_{\eta_{2}, \eta_{2}^{\prime}} \delta_{\eta_{3}, \eta_{3}^{\prime}} \\
\bar{\varepsilon} \Gamma^{a_{2}} \varepsilon^{\prime} & =-2 i c_{2} f_{2}\left(v_{2}\right)_{ \pm}^{a_{2}} \delta_{\eta_{1}, \eta_{1}^{\prime}} \delta_{\eta_{3}, \eta_{3}^{\prime}} \\
\bar{\varepsilon} \Gamma^{a_{3}} \varepsilon^{\prime} & =-2 i c_{3} f_{3}\left(v_{3}\right)_{ \pm}^{a_{3}} \delta_{\eta_{1}, \eta_{1}^{\prime}} \delta_{\eta_{2}, \eta_{2}^{\prime}} \\
\bar{\varepsilon} \Gamma^{a} \varepsilon^{\prime} & =0
\end{aligned}
$$

where $\left(v_{i}\right)_{ \pm}^{A}$, with $i=1,2,3$, and $A=0,1,2,3,4,5,6,7,8$ are the Killing vectors for the factors $A d S_{3}, S_{2}^{3}$, and $S_{3}^{3}$ with unit radius respectively, expressed in terms of frame indices $A$. To obtain the Killing vectors in the customary Einstein indices $M$, as in (D.2), it suffices to scale the Killing vectors $\left(v_{i}\right)_{ \pm}^{A}$ with frame indices, by the appropriate corresponding metric factors $f_{i}$ of (D.4). This rescaling precisely absorbs the prefactors $f_{i}$ in (D.17), but not the constants $c_{i}$.

The presence of the remaining factors $c_{i}$ reveals that the closure of the fermionic generators is precisely that of $D(2,1 ; c) \times D(2,1 ; c)$ with $c=c_{2} / c_{1}$. This may be seen by consulting [21], (or [71] page 201), for the precise structure relations of $D(2,1 ; c)$. Given that the maximal bosonic subalgebra of this superalgebra must be $S O(2,2) \times S O(4) \times S O(4)$, we find that, more precisely, the fermionic generators close onto the following real form, $D(2,1 ; c ; 0) \times D(2,1 ; c ; 0)$. 


\section{References}

[1] P. G. O. Freund and M. A. Rubin, "Dynamics Of Dimensional Reduction," Phys. Lett. B 97 (1980) 233.

[2] L. J. Romans, "New Compactifications Of Chiral N=2 D = 10 Supergravity," Phys. Lett. B 153 (1985) 392.

[3] J. Figueroa-O'Farrill and G. Papadopoulos, "Maximally supersymmetric solutions of ten- and eleven-dimensional supergravities," JHEP 0303 (2003) 048 [arXiv:hep-th/0211089].

[4] U. Gran, J. Gutowski, G. Papadopoulos and D. Roest, "IIB solutions with $N>28$ Killing spinors are maximally supersymmetric," JHEP 0712 (2007) 070 [arXiv:0710.1829 [hep-th]].

[5] H. Lin, O. Lunin and J. M. Maldacena, "Bubbling AdS space and 1/2 BPS geometries," JHEP 0410 (2004) 025 [arXiv:hep-th/0409174].

[6] D. Berenstein, "A toy model for the AdS/CFT correspondence," JHEP 0407, 018 (2004) [arXiv:hep-th/0403110].

[7] E. D'Hoker, J. Estes and M. Gutperle, "Exact half-BPS Type IIB interface solutions I: Local solution and supersymmetric Janus," JHEP 0706, 021 (2007) [arXiv:0705.0022 [hep-th]].

[8] E. D'Hoker, J. Estes and M. Gutperle, "Exact half-BPS Type IIB interface solutions. II: Flux solutions and multi-janus," JHEP 0706, 022 (2007) [arXiv:0705.0024 [hep-th]].

[9] E. D'Hoker, J. Estes and M. Gutperle, "Gravity duals of half-BPS Wilson loops," JHEP 0706 (2007) 063 [arXiv:0705.1004 [hep-th]].

[10] E. D'Hoker, J. Estes, M. Gutperle and D. Krym, "Exact Half-BPS Flux Solutions in M-theory I, Local Solutions," arXiv:0806.0605 [hep-th].

[11] J. P. Gauntlett, D. Martelli, S. Pakis and D. Waldram, "G-structures and wrapped NS5branes," Commun. Math. Phys. 247, 421 (2004) [arXiv:hep-th/0205050].

[12] J. P. Gauntlett and S. Pakis, "The geometry of D = 11 Killing spinors," JHEP 0304, 039 (2003) [arXiv:hep-th/0212008].

[13] J. P. Gauntlett, "Classifying supergravity solutions," Fortsch. Phys. 53, 468 (2005) [arXiv:hepth/0501229].

[14] J. P. Gauntlett, D. Martelli, J. Sparks and D. Waldram, "Supersymmetric AdS(5) solutions of M-theory," Class. Quant. Grav. 21, 4335 (2004) [arXiv:hep-th/0402153].

[15] I. Kirsch and D. Vaman, "The D3/D7 background and flavor dependence of Regge trajectories," Phys. Rev. D 72, 026007 (2005) [arXiv:hep-th/0505164].

[16] E. I. Buchbinder, J. Gomis and F. Passerini, "Holographic Gauge Theories in Background Fields and Surface Operators," JHEP 0712 (2007) 101 [arXiv:0710.5170 [hep-th]]. 
[17] J. A. Harvey and A. B. Royston, "Gauge/Gravity duality with a chiral $N=(0,8)$ string defect," arXiv:0804.2854 [hep-th].

[18] O. Aharony, A. Fayyazuddin and J. M. Maldacena, "The large N limit of N = 2,1 field theories from three-branes in F-theory," JHEP 9807 (1998) 013 [arXiv:hep-th/9806159].

[19] M. Grana and J. Polchinski, "Gauge / gravity duals with holomorphic dilaton," Phys. Rev. D 65 (2002) 126005 [arXiv:hep-th/0106014].

[20] H. J. Boonstra, B. Peeters and K. Skenderis, "Brane intersections, anti-de Sitter spacetimes and dual superconformal theories," Nucl. Phys. B 533 (1998) 127 [arXiv:hep-th/9803231].

[21] J. P. Gauntlett, R. C. Myers and P. K. Townsend, "Supersymmetry of rotating branes," Phys. Rev. D 59 (1999) 025001 [arXiv:hep-th/9809065].

[22] J. de Boer, A. Pasquinucci and K. Skenderis, "AdS/CFT dualities involving large 2d N = 4 superconformal symmetry," Adv. Theor. Math. Phys. 3 (1999) 577 [arXiv:hep-th/9904073].

[23] A. Van Proeyen, "Superconformal Algebras," http://www.slac.stanford.edu/spires/find/hep/www?irn=1943812 in Vancouver 1986, Proceedings, Super Field Theories, 547-555

[24] J. M. Maldacena, "The large N limit of superconformal field theories and supergravity," Adv. Theor. Math. Phys. 2, 231 (1998) [Int. J. Theor. Phys. 38, 1113 (1999)] [arXiv:hepth/9711200].

[25] S. S. Gubser, I. R. Klebanov and A. M. Polyakov, "Gauge theory correlators from non-critical string theory," Phys. Lett. B 428, 105 (1998) [arXiv:hep-th/9802109].

[26] E. Witten, "Anti-de Sitter space and holography," Adv. Theor. Math. Phys. 2, 253 (1998) [arXiv:hep-th/9802150].

[27] O. Aharony, S. S. Gubser, J. M. Maldacena, H. Ooguri and Y. Oz, "Large N field theories, string theory and gravity," Phys. Rept. 323, 183 (2000) [arXiv:hep-th/9905111].

[28] E. D'Hoker and D. Z. Freedman, "Supersymmetric gauge theories and the AdS/CFT correspondence," arXiv:hep-th/0201253.

[29] N. Drukker, D. J. Gross and H. Ooguri, "Wilson loops and minimal surfaces," Phys. Rev. D 60, 125006 (1999) [arXiv:hep-th/9904191].

[30] M. Bianchi, M. B. Green and S. Kovacs, "Instanton corrections to circular Wilson loops in N = 4 supersymmetric Yang-Mills," JHEP 0204, 040 (2002) [arXiv:hep-th/0202003].

[31] S. Gukov and E. Witten, "Gauge theory, ramification, and the geometric langlands program," arXiv:hep-th/0612073.

[32] S. Gukov and E. Witten, "Rigid Surface Operators," arXiv:0804.1561 [hep-th]. 
[33] N. Drukker, J. Gomis and S. Matsuura, "Probing N=4 SYM With Surface Operators," arXiv:0805.4199 [hep-th].

[34] J. Gomis and S. Matsuura, "Bubbling surface operators and S-duality," JHEP 0706 (2007) 025 [arXiv:0704.1657 [hep-th]].

[35] E. D'Hoker, J. Estes and M. Gutperle, "Interface Yang-Mills, supersymmetry, and Janus," Nucl. Phys. B 753 (2006) 16 [arXiv:hep-th/0603013].

[36] D. Gaiotto and E. Witten, "S-Duality of Boundary Conditions In N=4 Super Yang-Mills Theory," arXiv:0807.3720 [hep-th].

[37] D. Gaiotto and E. Witten, "Supersymmetric Boundary Conditions in N=4 Super Yang-Mills Theory," arXiv:0804.2902 [hep-th].

[38] D. Gaiotto and E. Witten, "Janus Configurations, Chern-Simons Couplings, And The ThetaAngle in N=4 Super Yang-Mills Theory," arXiv:0804.2907 [hep-th].

[39] N. R. Constable, J. Erdmenger, Z. Guralnik and I. Kirsch, "Intersecting D3-branes and holography," Phys. Rev. D 68 (2003) 106007 [arXiv:hep-th/0211222].

[40] P. Breitenlohner and D. Z. Freedman, "Positive Energy In Anti-De Sitter Backgrounds And Gauged Extended Supergravity," Phys. Lett. B 115 (1982) 197.

[41] K. Skenderis and M. Taylor, "Branes in AdS and pp-wave spacetimes," JHEP 0206 (2002) 025 [arXiv:hep-th/0204054].

[42] A. Karch and L. Randall, "Locally localized gravity," JHEP 0105, 008 (2001) [arXiv:hepth/0011156].

[43] A. Karch and L. Randall, "Open and closed string interpretation of SUSY CFT's on branes with boundaries," JHEP 0106, 063 (2001) [arXiv:hep-th/0105132].

[44] M. Cederwall, A. von Gussich, B. E. W. Nilsson, P. Sundell and A. Westerberg, "The Dirichlet super-p-branes in ten-dimensional type IIA and IIB supergravity," Nucl. Phys. B 490 (1997) 179 [arXiv:hep-th/9611159].

[45] M. Aganagic, C. Popescu and J. H. Schwarz, "D-brane actions with local kappa symmetry," Phys. Lett. B 393 (1997) 311 [arXiv:hep-th/9610249].

[46] E. Bergshoeff and P. K. Townsend, "Super D-branes," Nucl. Phys. B 490 (1997) 145 [arXiv:hep-th/9611173].

[47] J. Gomis and F. Passerini, "Holographic Wilson loops," JHEP 0608 (2006) 074 [arXiv:hepth/0604007].

[48] O. DeWolfe, D. Z. Freedman and H. Ooguri, "Holography and defect conformal field theories," Phys. Rev. D 66 (2002) 025009 [arXiv:hep-th/0111135]. 
[49] F J. Erdmenger, Z. Guralnik and I. Kirsch, "Four-dimensional superconformal theories with interacting boundaries or defects," Phys. Rev. D 66 (2002) 025020 [arXiv:hep-th/0203020].

[50] A. Karch and E. Katz, "Adding flavor to AdS/CFT," JHEP 0206, 043 (2002) [arXiv:hepth/0205236].

[51] J. H. Schwarz, "Covariant Field Equations Of Chiral N=2 D=10 Supergravity," Nucl. Phys. B 226 (1983) 269.

[52] S. Yamaguchi, "Bubbling geometries for half BPS Wilson lines," Int. J. Mod. Phys. A 22, 1353 (2007) [arXiv:hep-th/0601089].

[53] O. Lunin, "On gravitational description of Wilson lines," JHEP 0606, 026 (2006) [arXiv:hepth/0604133].

[54] J. Gomis, S. Matsuura, T. Okuda and D. Trancanelli, "Wilson loop correlators at strong coupling: from matrices to bubbling geometries," arXiv:0807.3330 [hep-th].

[55] T. Okuda and D. Trancanelli, "Spectral curves, emergent geometry, and bubbling solutions for Wilson loops," arXiv:0806.4191 [hep-th].

[56] J. Gomis and C. Romelsberger, "Bubbling defect CFT's," JHEP 0608, 050 (2006) [arXiv:hepth/0604155].

[57] J. Bagger and N. Lambert, "Comments On Multiple M2-branes," JHEP 0802 (2008) 105 [arXiv:0712.3738 [hep-th]].

[58] J. Bagger and N. Lambert, "Gauge Symmetry and Supersymmetry of Multiple M2-Branes," Phys. Rev. D 77 (2008) 065008 [arXiv:0711.0955 [hep-th]].

[59] A. Gustavsson, "Algebraic structures on parallel M2-branes," arXiv:0709.1260 [hep-th].

[60] O. Aharony, O. Bergman, D. L. Jafferis and J. Maldacena, "N=6 superconformal ChernSimons-matter theories, M2-branes and their gravity duals," arXiv:0806.1218 [hep-th].

[61] O. Aharony, M. Berkooz and N. Seiberg, "Light-cone description of $(2,0)$ superconformal theories in six dimensions," Adv. Theor. Math. Phys. 2 (1998) 119 [arXiv:hep-th/9712117].

[62] N. Kim and J. T. Yee, "Supersymmetry and branes in M-theory plane-waves," Phys. Rev. D 67 (2003) 046004 [arXiv:hep-th/0211029].

[63] O. Lunin, "1/2-BPS states in M theory and defects in the dual CFTs," JHEP 0710 (2007) 014 [arXiv:0704.3442 [hep-th]].

[64] E. Cremmer, B. Julia and J. Scherk, "Supergravity theory in 11 dimensions," Phys. Lett. B 76 (1978) 409.

[65] E. D'Hoker, J. Estes, M. Gutperle and D. Krym, "Exact Half-BPS Flux Solutions in M-theory II: Global solutions asymptotic to $A d S_{7} x S^{4}, "$ arXiv:0810.4647 [hep-th]. 
[66] P. S. Howe, N. D. Lambert and P. C. West, "The self-dual string soliton," Nucl. Phys. B 515 (1998) 203 [arXiv:hep-th/9709014].

[67] V. G. Kac, "A Sketch Of Lie Superalgebra Theory," Commun. Math. Phys. 53 (1977) 31.

[68] V. G. Kac, "Lie Superalgebras," Adv. Math. 26 (1977) 8.

[69] M. Scheunert, W. Nahm and V. Rittenberg, "Classification Of All Simple Graded Lie Algebras Whose Lie Algebra Is Reductive. 1," J. Math. Phys. 17, 1626 (1976).

[70] M. Scheunert, W. Nahm and V. Rittenberg, "Classification Of All Simple Graded Lie Algebras Whose Lie Algebra Is Reductive. 2. Construction Of The Exceptional Algebras," J. Math. Phys. 17, 1640 (1976).

[71] L. Frappat, A. Sciarrino, and P. Sorba, Dictionary on Lie Algebras and Superalgebras, Academic Press, 2000.

[72] M. Parker, "Classification Of Real Simple Lie Superalgebras Of Classical Type," J. Math. Phys. 21 (1980) 689.

[73] I. Bars and M. Gunaydin, "Unitary Representations Of Noncompact Supergroups," Commun. Math. Phys. 91, 31 (1983).

[74] M. Gunaydin and N. P. Warner, "Unitary Supermultiplets Of Osp $(8 / 4, R)$ And The Spectrum Of The S(7) Compactification Of Eleven-Dimensional Supergravity," Nucl. Phys. B 272, 99 (1986).

[75] M. Gunaydin and R. J. Scalise, "Unitaru Lowest Weight Representations Of The Noncompact Supergroup $\operatorname{OSp}\left(2 \mathrm{~m}^{*} / 2 \mathrm{n}\right)$," J. Math. Phys. 32, 599 (1991).

[76] J. D. Brown and M. Henneaux, "Central Charges in the Canonical Realization of Asymptotic Symmetries: An Example from Three-Dimensional Gravity," Commun. Math. Phys. 104, 207 (1986).

[77] M. Henneaux and C. Teitelboim, "Asymptotically Anti-de Sitter Spaces", Commun. Math. Phys. 98 (1985) 391.

[78] S. Hollands, A. Ishibashi and D. Marolf, "Comparison between various notions of conserved charges in asymptotically AdS-spacetimes," Class. Quant. Grav. 22, 2881 (2005) [arXiv:hepth/0503045].

[79] K. Skenderis, "Lecture notes on holographic renormalization," Class. Quant. Grav. 19 (2002) 5849 [arXiv:hep-th/0209067].

[80] L. F. Abbott and S. Deser, "Stability Of Gravity With A Cosmological Constant," Nucl. Phys. B 195, 76 (1982).

[81] S. Helgason, Differential Geometry, Lie groups, and Symmetric Spaces, Academic Press, 1978

[82] A. Besse, Einstein manifolds, Springer-Verlag (1986). 
[83] S. Kobayashi and K. Nomizu, Foundations of Differential Geometry, Vol II, John Wiley Interscience (1969).

[84] H. Lin and J. M. Maldacena, "Fivebranes from gauge theory," Phys. Rev. D 74 (2006) 084014 [arXiv:hep-th/0509235].

[85] G. van Anders, "General Lin-Maldacena solutions and PWMM instantons from supergravity," JHEP 0703, 028 (2007) [arXiv:hep-th/0701277].

[86] H. H. Shieh, G. van Anders and M. Van Raamsdonk, "Coarse-Graining the Lin-Maldacena Geometries," JHEP 0709 (2007) 059 [arXiv:0705.4308 [hep-th]].

[87] E. D'Hoker and Y. Guo, in preparation.

[88] J. Gomis and F. Passerini, "Wilson loops as D3-branes," JHEP 0701, 097 (2007) [arXiv:hepth/0612022].

[89] N. Kim, "AdS(3) solutions of IIB supergravity from D3-branes," JHEP 0601, 094 (2006) [arXiv:hep-th/0511029].

[90] J. P. Gauntlett, N. Kim and D. Waldram, "Supersymmetric AdS(3), $\operatorname{AdS}(2)$ and bubble solutions," JHEP 0704, 005 (2007) [arXiv:hep-th/0612253].

[91] M.M. Sheikh-Jabbari, unpublished.

[92] J. M. Figueroa-O'Farrill, "Deformations of M-theory Killing superalgebras," Class. Quant. Grav. 24, 5257 (2007) [arXiv:0706.2600 [hep-th]].

[93] N. Kim and J. D. Park, "Comments on AdS(2) solutions of D = 11 supergravity," JHEP 0609, 041 (2006) [arXiv:hep-th/0607093].

[94] O. A. P. Mac Conamhna and E. O Colgain, "Supersymmetric wrapped membranes, AdS(2) spaces, and bubbling geometries," JHEP 0703, 115 (2007) [arXiv:hep-th/0612196].

[95] J. P. Gauntlett, O. A. P. Mac Conamhna, T. Mateos and D. Waldram, "New supersymmetric AdS(3) solutions," Phys. Rev. D 74, 106007 (2006) [arXiv:hep-th/0608055].

[96] P. Figueras, O. A. P. Mac Conamhna and E. O Colgain, "Global geometry of the supersymmetric AdS(3)/CFT(2) correspondence in Phys. Rev. D 76, 046007 (2007) [arXiv:hep-th/0703275].

[97] B. E. W. Nilsson and C. N. Pope, "Hopf Fibration Of Eleven-Dimensional Supergravity," Class. Quant. Grav. 1, 499 (1984).

[98] I. Klebanov, T. Klose and A. Murugan, " $A d S_{4} / C F T_{3}$ - Squashed, Stretched and Warped," arXiv:0809.3773 [hep-th].

[99] L. Frappat, A. Sciarrino and P. Sorba, "Structure of Basic Lie Superalgebras and of their Affine Extensions", Commun. Math. Phys. 121 (1989) 457. 